\title{
Do black holes radiate?
}

\author{
Adam D Helfer \\ Department of Mathematics, Mathematical Sciences Building, \\ University of Missouri, Columbia, Missouri 65211, U.S.A.
}

\begin{abstract}
The prediction that black holes radiate due to quantum effects is often considered one of the most secure in quantum field theory in curved space-time. Yet this prediction rests on two dubious assumptions: that ordinary physics may be applied to vacuum fluctuations at energy scales increasing exponentially without bound; and that quantum-gravitational effects may be neglected. Various suggestions have been put forward to address these issues: that they might be explained away by lessons from sonic black hole models; that the prediction is indeed successfully reproduced by quantum gravity; that the success of the link provided by the prediction between black holes and thermodynamics justifies the prediction.

This paper explains the nature of the difficulties, and reviews the proposals that have been put forward to deal with them. None of the proposals put forward can so far be considered to be really successful, and simple dimensional arguments show that quantum-gravitational effects might well alter the evaporation process outlined by Hawking. Thus a definitive theoretical treatment will require an understanding of quantum gravity in at least some regimes. Until then, no compelling theoretical case for or against radiation by black holes is likely to be made.

The possibility that non-radiating "mini" black holes exist should be taken seriously; such holes could be part of the dark matter in the Universe. Attempts to place observational limits on the number of "mini" black holes (independent of the assumption that they radiate) would be most welcome.
\end{abstract}

PACS number(s): 04.70.Dy, 04.60.-m, 97.60.Lf

Date: 7 February 2008 


\section{Contents}

1. Introduction

2. The predictions

3. The Hawking process

3.1 The space-time

3.2 Null infinity, the black hole, and time-asymmetry

3.3 The mapping of surfaces of constant phase

3.4 Propagation of the quantum field

3.5 The quantum state and the two-point functions

3.5.1 The two-point function in Minkowski space

3.5.2 The two-point function in the Hawking model

3.5.3 Particles

3.6 Stress-energy

3.7 The analysis of Fredenhagen and Haag

3.8 Almost-black holes

4. The trans-Planckian problem

4.1 Localization of the problem

4.1.1 The family $(\mathrm{CH})$

4.1.2 The family (SO)

4.2 Discussion

5. Connection with the Unruh process

5.1 The Unruh process

5.2 The argument from the equivalence principle

5.3 The Bisognano-Wichmann theorem

6. Lessons from moving-mirror models

6.1 The trajectories

6.2 The energy budget

7. Connections with thermodynamics

7.1 Classical black-hole thermodynamics

7.2 General relativity and the second law

7.3 The generalized second law

7.4 The generalized second law and the Hawking process

7.4.1 Quantum-gravitational issues

7.4.2 Definition of entropy

7.4.3 Rate of entropy production

7.5 The Geroch-Wheeler process revisited 
Contents (continued)

8. Nonstandard propagation

8.1 Jacobson's cut-off model

8.2 The model of Corley and Jacobson

8.2.1 The space-times

8.2.2 The dispersive propagation

8.2.3 Quantization

8.3 The model of Brout, Massar, Parentani and Spindel

8.4 Summary and discussion

9. 't Hooft's $S$-matrix black hole theory

9.1 Overview of the program

9.1.1 The spectrum

9.1.2 The holographic principle

9.1.3 The trans-Planckian problem

9.1.4 The $S$-matrix theory

9.1.5 Purity and unitarity

9.2 Summary

10. Evidence from theories of quantum gravity

10.1 Dilatonic black holes

10.2 TTFKAS

10.3 Ashtekar's approach

10.4 Euclidean quantum gravity

11. Quantum character of space-time

11.1 Validity of the semiclassical approximation

11.2 Quantization of black-hole area

11.3 Quantum measurement issues

12. Experimental prospects

12.1 What sorts of black holes might exist?

12.2 What would a Hawking-radiating black hole really look like?

12.3 Summary

13. Conclusions

Appendix. Text passages supporting the conclusions 


\section{Introduction}

In 1974 and 1975, Stephen Hawking published his analysis of the effects of gravitational collapse on quantum fields, and predicted that black holes are not in fact black, but radiate thermally and eventually explode. Whether black holes turn out to radiate or not, it would be hard to overstate the significance of these papers. Hawking had found one of those key physical systems which at once bring vexing foundational issues to a point, are accessible to analytic techniques, and suggest deep connections between disparate areas of physics.

Work stimulated by Hawking's led to clarifications of what it means to detect or create particles, and even of what it means to define a quantum field theory. Perhaps more excitingly, Hawking's was a concrete proposal that quantum effects might qualitatively alter the character of general relativity, turning black holes into sources rather than perfect sinks. This mechanism, involving in essential ways as it did quantum theory and general relativity, suggested that the system might be a point of entry to the problem of quantizing gravity. And it suggested that the connections between black holes and thermodynamics, which until that time had appeared to most workers to be formal (see however Bekenstein 1973) were real and might be understood quantum-theoretically.

Given the profound nature of the issues addressed, it is perhaps not surprising that some disagreement and controversy exists over exactly what has been achieved. Thus some authors write that the arguments for thermal radiation are almost certainly secure (Carlip 2001), others that there are problems but that those can almost certainly be fixed (Wald 2001), or that there are problems which probably can be fixed (Jacobson 1990), or that there are problems which hopefully can be fixed (Parentani 2001), or that there are problems which may not be fixable (Belinski 1995). According to some, the prediction depends on speculations about ultra-high energy physics (Gibbons 1977); according to others, this has been shown not to be the case (Visser 2001). Most workers assume a priori that quantum-gravitational effects will be negligible, since there are no locally Planck-scale curvatures (except perhaps for holes of around the Planck mass), but others have argued that quantum-gravitational effects could completely alter or even obviate the thermal radiation (Bekenstein and Mukhanov 1995, Ashtekar 1998). There are those who feel that the case for Hawking radiation is so compelling that it should be a touchstone for theories of quantum gravity: we should grade the plausibility of theories of quantum gravity according to whether they reproduce Hawking's predictions (Carlip 2001) — which should be contrasted with grading the plausibility of the predictions according to whether they are sustained by theories of quantum gravity.

How confident should we be in the prediction of thermal radiation from black holes? Is the evidence really good enough that we can pass or reject theories of quantum gravity (or of links between thermodynamics and quantum fields in curved space-time) on the basis of it? If the evidence is not that good, are we potentially 
selecting against the correct theory of quantum gravity (or the correct understanding of the link with thermodynamics) by only considering theories compatible with the predictions? To what extent does the proposed mechanism really probe quantum gravity?

It is these questions which are the subject of the present review. So this paper is not a pedagogical treatment of a well-understood area, but a critical review of the current state of a difficult issue. The emphasis will be on a clear presentation of the physical issues and their interplay.

The main conclusions may be summarized as follows: ${ }^{1}$

(a) None of the derivations that have been given of the prediction of radiation from black holes is convincing. All involve, at some point, speculations of what physics is like at scales which are not merely orders of magnitude beyond any that have so far been investigated experimentally $\left(\sim 10^{3} \mathrm{GeV}\right)$, but at and increasing beyond the Planck scale $\left(\sim 10^{19} \mathrm{GeV}\right)$, where essentially quantum-gravitational effects are expected to be dominant. (In Hawking's treatment, this increase occurs exponentially quickly.) Some of these speculations may be plausible, but none can be considered reliable. ${ }^{2}$

(b) There are equally plausible speculations about physics at such scales which result in no radiation at all, or in non-thermal spectra.

(c) The various derivations which have been put forward are not all mutually consistent. Thus, even among the derivations which do give rise to thermal radiation, there is no single accepted physical mechanism.

(d) Quantum-gravitational corrections are very plausibly of a size to completely alter or even obviate the prediction of thermal radiation.

(e) A number of the arguments put forward in support of the Hawking mechanism are not really direct evidence for the existence of thermal radiation, but rather are arguments for interpreting black holes' areas as entropies.

(f) The proposed mechanism, at least as conventionally understood, relies precisely on the assumption that quantum-gravitational effects can be neglected, and so no deep test of quantum gravity can emerge from it.

It should be emphasized that the problems uncovered here are entirely physical, not mathematical. While there are some technical mathematical concerns with details of Hawking's computation, we do not anticipate any real difficulty in resolving these (cf. Fredenhagen and Haag 1990). The issues are whether the physical assumptions underlying the mathematics are correct, and whether the correct physical lessons are being drawn from the calculations.

The significance of these conclusions is not negative, however, but positive and exciting. They show that the precise assumptions we make about certain aspects of quantum gravity and high-energy physics do have an effect on Hawking's predictions.

${ }^{1}$ The appendix lists the places in the text where these assertions are justified.

${ }^{2}$ It should be remarked that very similar issues arise in contemporary inflationary cosmology. See e.g. Brandenberger 2002 and references therein. 
So the challenge to any theory of quantum gravity is (not necessarily to reproduce Hawking's predictions, but) to provide a detailed physical picture of what happens to quantum fields in a region where a black hole forms. Different theories will lead to different pictures, more or less plausible and with differing implications. Even at the theoretical level, the requirement of being able to produce a plausible and selfconsistent model is a terrific constraint. And of course the sensitivity to assumptions means that, should experimental data become available, we can discriminate between different theories.

It should also be noted that the issues that occur in the black-hole case - the appearance of trans-Planckian modes, and the question of quantum-gravitational corrections - are also of interest in contemporary inflationary cosmology. (See e.g. Brandenberger 2002.)

A few historical comments may help to orient the reader.

The trans-Planckian problem and the question of quantum-gravitational corrections were apparently recognized almost immediately on the publication of Hawking's work; for example, they are raised in Gibbons's (1977) paper. However, for some reason (which is to me puzzling), these issues did not get much attention for a long time. They were Unruh's main motivation for introducing the idea of sonic black holes (Unruh 1981); he clearly considered the issues serious, but other workers did not pursue them. Jacobson $(1990,1991)$ gave what is as far as I know the first explicit statement of the problem since Gibbons's and Unruh's papers.

Jacobson's (1991) paper was very influential in one sector of the relativity community (it made the LANL "top cite" list). He carefully explained the transPlanckian problem, and then suggested a template for a scheme to "save" the Hawking prediction without appealing to trans-Planckian physics. Then several papers by Jacobson and Unruh developed an idea that if new physics intervened and the propagation of field modes at high frequencies had a certain dispersive character, one could recover Hawking's prediction (by, however, a mechanism essentially different from that envisaged by Hawking). This mechanism still required trans-Planckian wave-numbers.

At this point, a chronological account becomes of limited value, because, while the papers of Jacobson and Unruh did attract attention, there was no unanimity about what lessons were to be drawn from them. Had the trans-Planckian problem been solved? If not, how serious was the situation? Even if the issue was still open and serious, surely the connections which had been established between the Hawking effect and other physics must be good circumstantial evidence that the prediction of blackhole evaporation is in fact correct? Physics (and particularly quantum field theory) has some spectacular examples of the right answers gotten for the wrong reasons. Perhaps that is the case here?

That is more or less where we are today. In recent years, a few papers have been appearing with cautionary remarks that there are unresolved difficulties with the mechanism proposed by Hawking. Yet most workers seem uncertain about just 
how serious those difficulties are. The aim of this paper is to lay out the status of our understanding so that they may judge for themselves. For earlier perspectives, see Brout et al (1995a), Jacobson (1999).

The organization of the paper is this.

Section 2 is a brief overview of Hawking's predictions, before explaining the theory behind them. The emphasis is on understanding the scales of the predicted effects. Section 3 is a review of the mechanism as it is usually understood. The emphasis is on making clear the nature of the physical assumptions, rather than on details of the computations. Section 4 discusses the trans-Planckian problem (the occurrence of arbitrarily high energy scales), and the sense in which it is localized.

Sections 5-10 cover the arguments that have been put forward in support of the Hawking process. These include an attempt to derive it from a combination of the Unruh effect and the principle of equivalence, connections between it and movingmirror models, arguments that it can be derived from or essentially links general relativity and thermodynamics, attempts to avoid the trans-Planckian problem by introducing non-standard rules for the propagation of the quantum field, and arguments in support of the Hawking mechanism from quantum theories of gravity. 't Hooft's program for analyzing the quantum structure of black holes is discussed in section 9.

Section 11 takes up quantum-gravitational issues from another direction. Rather than examining the consequences of particular theories of quantum gravity (strings, loops, etc.), it considers the general physical consequences which might be expected to arise from any sort of quantum character of space-time. These include quantum limitations on measurement, discreteness of eigenvalues, and so on.

Section 12 discusses the experimental prospects for resolving the question, and section 13 is a brief summary of the conclusions. An appendix lists the points in the text supporting the main conclusions.

Conventions. In most places in this paper, factors of $c, \hbar, G$ and $k$ (Boltzmann's constant) are given explicitly, but in a few places, where it would be too cumbersome, factors of $c$ are omitted. As has become conventional in this area, a factor of $k^{-1}$ is absorbed in the definition of entropies, so that they are pure numbers. The conventions for general relativity are those of Penrose and Rindler (1984-6), and for quantum field theory are those of Schweber (1961). The metric signature is + - - - The Planck length, Planck time, Planck energy and Planck mass are

$$
\begin{aligned}
l_{\mathrm{Pl}} & =\left(G \hbar / c^{3}\right)^{1 / 2} \simeq 1.6 \cdot 10^{-33} \mathrm{~cm} \\
t_{\mathrm{Pl}} & =\left(G \hbar / c^{5}\right)^{1 / 2} \simeq 5.4 \cdot 10^{-44} \mathrm{~s} \\
E_{\mathrm{Pl}} & =\left(\hbar c^{5} / G\right)^{1 / 2} \simeq 1.2 \cdot 10^{19} \mathrm{GeV} \\
m_{\mathrm{Pl}} & =(\hbar c / G)^{1 / 2} \simeq 2.2 \cdot 10^{-5} \mathrm{~g} .
\end{aligned}
$$




\section{The predictions}

This section gives a brief overview of Hawking's predictions in the case of linear quantum fields, and without discussing their derivations. The aim is to acquaint the reader with the physical scales involved. We shall see that the Hawking effect would be (in most circumstances, and also in a certain scale-invariant sense) extremely tiny. One will need to think carefully about all possible physical effects at or above these scales in order to have confidence that black holes radiate.

Hawking's predictions apply to isolated black holes which formed by gravitational collapse but have settled down to (macroscopically) stationary states. The assumption that the holes formed by collapse (rather than were created with the Universe) is essential, as will be discussed in section 3.4. According to the "black hole uniqueness" theorems, each isolated, stationary black hole which occurs in Nature should be characterized by its mass $M$, angular momentum $J$ and electric charge $Q$; its exterior will be a Kerr-Newman solution of Einstein's equation. Since we are interested in a critical assessment of the theory, we consider for the most part the simplest case, an uncharged, spherically symmetric hole (so $Q=0$ and $J=0$ ), whose exterior is Schwarzschild. The difficulties are already apparent in this case.

A key quantity in black-hole physics is the surface gravity $\kappa$, which may be defined for example as the acceleration measured by red-shifts of light rays passing close to the horizon (Helfer 2001b). For a Schwarzschild black hole, one has

$$
\kappa=c^{4} /(4 G M) .
$$

Since the Schwarzschild radius is $R_{\mathrm{Sch}}=2 G M / c^{2}$, one can think of $c / \kappa=2 R_{\mathrm{Sch}} / c$ as the light-crossing time of the hole. This will be an important time scale in later sections. Classically, this is the time scale for the final approach to the black-hole state (which occurs exponentially quickly). In the Hawking process, this is the timescale for an exponential blue-shift in the frequencies of the particular vacuum modes which give rise to the Hawking quanta at any time. Numerically, one has

$$
c / \kappa \simeq 2.0 \cdot 10^{-5}\left(M / M_{\odot}\right) \mathrm{s},
$$

where $M_{\odot}$ is the mass of the Sun. Thus for solar-mass or smaller black holes the time scale is very short by ordinary standards. Even for a super-massive black hole of size $\sim 10^{8} M_{\odot}$, the time scale would only be about half an hour.

According to Hawking, a free massless field will radiate at a temperature

$$
T_{\mathrm{H}}=(\hbar / 2 \pi c k) \kappa .
$$

(Of course, we do not know of any free fields in Nature - all known fields interact. The question of how Hawking's predictions might be modified by interactions (and mass) will be taken up in Section 12.2.) For a Schwarzschild hole, one has

$$
\begin{aligned}
T_{\mathrm{H}} & =\hbar c^{3} /(8 \pi G M k) \\
& \simeq 6.2 \cdot 10^{-8}\left(M_{\odot} / M\right) \mathrm{K} .
\end{aligned}
$$


Thus radiation from a solar-mass black hole would be exceedingly cold - about $5 \cdot 10^{7}$ times colder than the cosmic microwave background. Larger black holes would be colder still. This gives one a sense of just how easily Hawking radiation can be lost in other, apparently small, effects. Of course, smaller black holes would have higher temperatures, and there is some possibility that "mini" black holes might exist and that Hawking radiation from them might be detected. A "mini" black hole of mass $\sim 10^{15} \mathrm{~g}$ would have $T_{\mathrm{H}} \sim 10^{11} \mathrm{~K}$.

The luminosity of the black hole can be estimated from the Stefan-Boltzmann law if one has a measure of the effective radiating area. Precise calculations of this are lengthy, but their results are that the area is $\alpha A$, where $\alpha$ is a numerical factor of order unity and $A$ is the area of the hole. Thus for a Schwarzschild hole the luminosity due to a given massless field is

$$
L=\left(\pi^{2} k^{4} / 60 \hbar^{3} c^{2}\right) \alpha A T_{\mathrm{H}}^{4}
$$

(The precise Stefan-Boltzmann factor depends on the field species, but we shall absorb variances in this into $\alpha$.)

Notice that what one has is a black body with a cavity size of the same order as the dominant wavelength - both are $\sim R_{\text {Sch }}$. This corresponds to an object which is very dim in an invariant sense. While the spectrum is indeed thermal, it is at such a low temperature compared to its physical dimensions that it cannot be thought of very accurately as a classical flux of radiation. In fact, a measure of the time between emission of quanta may be given by

$$
\text { (mean energy per quantum) } / L=240 \pi^{-1} \alpha^{-1} \hbar /\left(k T_{\mathrm{H}}\right),
$$

that is, somewhat longer than the order of the mean period of the quanta. The emission of Hawking radiation from the black hole is thus a process which not only has a quantum origin but is quantum in its presentation.

Despite this essentially quantum character of the radiation, it is natural to expect that we should in a time-averaged sense be able to talk about the rate of mass loss of the hole due to the process. This is on its face a very natural suggestion, and seems to rely only on the assumption that energy is conserved. Then we have

$$
\frac{\mathrm{d} M}{\mathrm{~d} t}=-L / c^{2}
$$

for a Schwarzschild hole

$$
\frac{\mathrm{d} M}{\mathrm{~d} t}=-\left(\alpha c^{4} \hbar / 960 G^{2}\right) n_{\mathrm{eff}} M^{-2}
$$

where $n_{\text {eff }}$ is the effective number of radiating species. This number will depend (weakly) on $M$, since (for example) as the mass decreases, the temperature rises and linear fields of masses $\lesssim k T_{\mathrm{H}} / c^{2}$ will contribute significantly to the Hawking process. However, ignoring this dependence, we may get a rough estimate of the time scale 
over which the mass changes significantly due to the Hawking process (which will also be an estimate of the lifetime of the hole) by solving equation (8):

$$
t_{\text {life }} \sim\left(320 G^{2} / \alpha \hbar c^{4} n_{\text {eff }}\right) M^{3} \sim\left(M / M_{\odot}\right)^{3} \cdot 10^{65} \mathrm{y} .
$$

We are not interested in speculating about physics over scales longer than the age of the Universe. Our interest in this equation is rather that it constrains the possible masses of "mini" black holes generated early in the Universe. If we set $t_{\text {life }}$ to the present age of the Universe, we obtain a minimum mass such a "primordial" black hole must have had (assuming it Hawking-radiates) to survive to the present day. This mass is $\sim 10^{15} \mathrm{~g}$.

\section{The Hawking process}

In this section, I outline the derivation of the prediction of thermal radiation. I have kept the treatment of the essential physical elements of the mechanism quite close to Hawking's, but I have taken advantage of insights which have developed in the interim to streamline the presentation and avoid most of the technical mathematics. ${ }^{3}$

In outline, we have a scattering problem to solve: given an initial state of the quantum field, before the black hole has formed, find what the final state of the field will be, after the hole has formed (and any transients have passed). The underlying assumption will be that we can treat space-time classically, and the quantum fields as propagating on this background space-time.

\subsection{The space-time}

So let us begin by describing the space-time, $\left(\mathcal{M}, g_{a b}\right)$. We will assume it is spherically symmetric, and contains matter imploding to form a black hole. We also assume that this is isolated, and model this mathematically by assuming that the space-time is asymptotically flat. (It should be emphasized that in physical terms "asymptotic" here means a regime around the collapsing system in which spacetime is suitably flat; at late times, when the matter is very nearly collapsed, this might be anything from tens of Schwarzschild radii outwards. It is a convenient mathematical fiction to assume that the collapsing object is perfectly isolated, so that this asymptotically flat regime extends to infinity, and we shall do so, but one can verify that the physics does not depend on this.)

${ }^{3}$ Actually, Hawking's (1975) paper contained some important comments on the quantum character of space-time. These ideas have fallen out of fashion, and will not be discussed in this section, but they are close to concerns to be raised in section 11 , below. 


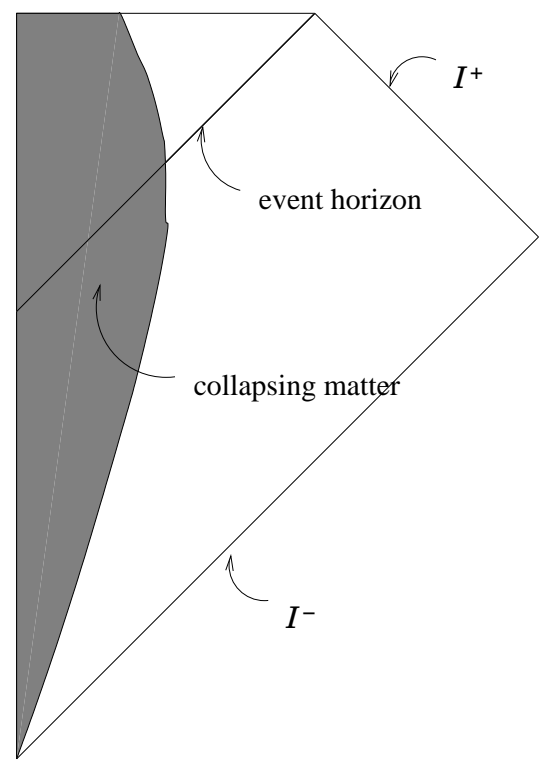

Figure 1. The Penrose diagram of a spherically symmetric object collapsing to form a black hole. The future and past null infinities $\mathcal{I}^{ \pm}$are shown, as well as the event horizon. The black hole is the region at and above the event horizon.

The standard Penrose diagram for this space-time is shown in figure 1, and the reader will find it convenient to refer to this. In this diagram, the rotational symmetry has been factored out, so each point represents a sphere of symmetry. Scales are distorted in the diagram, but the causal structure is accurately portrayed. Thus the lines at 45 degrees are null (and correspond to radial null geodesics in the space-time). The diagram also allows one to represent various infinite regimes as finite ones.

Since by Birkhoff's theorem a vacuum spherically symmetric space-time must be locally isometric to part of the extended Schwarzschild solution, we have an explicit understanding of the metric in the exterior of the matter. The Schwarzschild metric in standard coordinates is

$\mathrm{d} s^{2}=\left(1-\left(R_{\mathrm{Sch}} / r\right)\right) \mathrm{d} t^{2}-\left(1-\left(R_{\mathrm{Sch}} / r\right)\right)^{-1} \mathrm{~d} r^{2}-r^{2}\left(\mathrm{~d} \theta^{2}+\sin ^{2} \theta \mathrm{d} \varphi^{2}\right)$,

where $R_{\text {Sch }}=2 G M / c^{2}$ is the Schwarzschild radius and $M$ is the mass. It is also convenient to introduce the "tortoise" coordinate

$$
r_{*}=r-R_{\mathrm{Sch}}+R_{\mathrm{Sch}} \log \left(\left(r / R_{\mathrm{Sch}}\right)-1\right),
$$

as well as the retarded and advanced time coordinates

$$
u=t-r_{*}, \quad v=t+r_{*} .
$$

These are both null coordinates. The metric (10) and the coordinates are valid where both: (a) the actual metric is Schwarzschild (that is, exterior to the matter); and (b) one is outside the Schwarzschild radius.

We shall not need to consider in any detail the region of space-time within the black hole (that is, within the event horizon). We will need, however, some basic 
information about the portion of space-time containing matter and exterior to the hole, since the field modes which are ultimately supposed to give rise to Hawking radiation must propagate through this region first.

While the explicit form of the metric in this region will not be necessary, we can see that the coordinates $r, u, v, \theta$ and $\varphi$ have well-defined extensions to this region. The coordinate $r$ may be defined throughout the space-time by taking, for any event $p$, the area of the sphere of symmetry through $p$ to be $4 \pi r(p)^{2}$. The coordinates $u$ and $v$ may be extended by requiring them to remain null and spherically symmetric. They are good coordinates in the region under consideration except on the axis of symmetry. The angular coordinates $\theta$ and $\varphi$ may be extended unambiguously (except for the usual spherical coordinate singularities) by flowing in the two-spaces orthogonal to the spheres of symmetry.

\subsection{Null infinity, the black hole, and time-asymmetry}

A few words are in order at this point about the null asymptotic structure of the space-time, since this enters both in the specification of the initial and final data for the massless field and in the definition of the black hole. We may attach to space-time future and past null infinities $\mathcal{I}^{ \pm}$, which are null hypersurfaces. Each point on $\mathcal{I}^{+}$is reached by holding $(u, \theta, \varphi)$ constant and taking $r \rightarrow+\infty$; we take as usual $(u, \theta, \varphi)$ as coordinates on $\mathcal{I}^{+}$. As defined here, then, future null infinity consists of the future end-points of the radial null geodesics in the Schwarzschild exterior. Actually, every null geodesic which escapes (as one moves into the future) the gravitational pull of the matter has a well-defined end-point on $\mathcal{I}^{+}$. By exchanging "future" and "past," and $u$ and $v$, one has corresponding statements for $\mathcal{I}^{-}$.

The treatment of $\mathcal{I}^{+}$and $\mathcal{I}^{-}$has so far been symmetric, but the discussion of black holes breaks this symmetry. A black hole is defined to be a region of space-time from which signals cannot escape to arbitrarily distant regions. Formally, escape in this sense means reaching $\mathcal{I}^{+}$. Thus the black hole (if it forms) is the set of events in space-time for which there are no future-directed causal curves reaching $\mathcal{I}^{+}$. ${ }^{4}$ The boundary of this set is the future event horizon $\mathcal{H}^{+}$.

One could time-reverse the concept of a black hole; the result would be a white hole, a region which into which signals originating from very great distances could not penetrate, but from which signals might escape. Such an object would evidently be quite different from a black hole.

Note that this means that if a black-hole space-time is time-symmetric, it must also be a white-hole space-time. Such space-times are not models of gravitational collapse of isolated objects and are not of direct interest in the Hawking mechanism. The mechanism requires a time-asymmetric space-time.

\subsection{The mapping of surfaces of constant phase}

A key role will be played by the mapping of surfaces of constant phase. A surface

${ }^{4}$ A causal curve is one whose tangent is everywhere null or timelike (if it is differentiable). 
$u=$ constant is a spherically symmetric outgoing null hypersurface. If the radial null geodesics forming this surface are traced backwards in time through the spatial origin, they emerge to form a spherically null hypersurface which (if read forwards in time) would be incoming, that is, would be a $v=$ constant surface. Thus to each retarded time $u$ we may associate an advanced time $v(u)$. Since the $u=$ constant and $v=$ constant surfaces are the spherically symmetric surfaces of constant phase in the geometric-optics approximation, the function $v(u)$ is the mapping of surfaces of constant phase.

(Below, we shall need to refer both to the function $v(u)$ and to the coordinates $u(p), v(p)$ of an event $p$. These are quite different functions, with $v(u)$ an ordinary real-valued function of one real variable, but $v(p)$ and $u(p)$ each a real-valued function of the event $p$ in space-time. One could alternatively write something like $V(u)$ for the function $v(u)$, but this introduces an undesirable asymmetry between $u$ and $v$.)

Suppose a spherically symmetric massless wave is sent through the space-time, and the period is initially $\delta v$. In the geometric-optics approximation, it will emerge with period $\delta u$, where $\delta v / \delta u=\mathrm{d} v(u) / \mathrm{d} u$. This is the ratio of the frequencies, and so $\mathrm{d} v(u) / \mathrm{d} u$ is precisely the red-shift factor.

The mapping of surfaces of constant phase is intimately bound up with the formation of a black hole. It can be shown on very general grounds that when matter collapses to form a black hole, one has $\mathrm{d} v / \mathrm{d} u \rightarrow 0$ and the asymptotic relation

$$
\frac{\mathrm{d}^{2} v}{\mathrm{~d} u^{2}}=-\kappa \frac{\mathrm{d} v}{\mathrm{~d} u}+O\left((\mathrm{~d} v / \mathrm{d} u)^{2}\right) \quad \text { as } \quad u \rightarrow+\infty
$$

where $\kappa$ is the surface gravity of the hole (Helfer 2001). Notice that this is a universal relation, depending only on the surface gravity and not on any details of the formation of the hole. It implies

$$
\frac{\mathrm{d} v}{\mathrm{~d} u} \sim \exp -\kappa u \quad \text { as } u \rightarrow+\infty,
$$

so signals from the hole-to-be are exponentially red-shifted. Also one must have

$$
\lim _{u \rightarrow+\infty} v(u)=v_{0}
$$

where $v_{0}$ is the advanced time of formation of the hole, the advanced time at which the event horizon forms. We have

$$
v_{0}-v(u) \sim \exp -\kappa u \quad \text { as } u \rightarrow+\infty
$$

The details of the formulas $(14,16)$ will be absolutely central to the arguments for the Hawking process. This should be contrasted with the significances of the formulas for classical physics. Classically, the equations imply that (given that a horizon is about to form), there is a fast (time scale $\sim c \kappa^{-1}$ ) approach to the black-hole state, a state where $v(u)$ is indistinguishable from $v_{0}$ and $\mathrm{d} v / \mathrm{d} u$ is indistinguishable from zero. In distinction, the derivation of the Hawking process will rely on the literal 
validity of $(14,16)$ for arbitrarily late retarded times $u$. Thus the exponential increase of the red-shift, for arbitrarily long times, will be a central assumption of Hawking's argument.

Another important feature of the mapping of surfaces of constant phase is that it gives us a way of resolving the singularity of the coordinate $u$ at the event horizon. (At the event horizon, we have $u \rightarrow+\infty$.) If for any event $p$, we take the past-directed radial null geodesic inwards, let it pass through the spatial origin and then outwards to $\mathcal{I}^{-}$, it arrives with an advanced time $\tilde{v}(p)$. (The tilde is to distinguish $\tilde{v}(p)$ from the coordinate $v(p)$.) If $p$ lies before the event horizon, so that its retarded time $u(p)$ is well-defined, then $\tilde{v}(p)=v(u(p))$, where $v(u)$ is the mapping of surfaces of constant phase and $u(p)$ is the value of the coordinate $u$ at $p$. However, the coordinate $\tilde{v}$ is easily seen to be a good coordinate throughout space-time (except at the spatial origin), and by its definition is a coordinate constant on radial outgoing null surfaces.

Thus $v(u(p))=\tilde{v}(p)$, where $v(u)$ is the mapping of surfaces of constant phase, provides a good coordinate which extends naturally past the horizon. In the case of Schwarzschild space-time, this coordinate would be (up to a constant factor) the usual null Kruskal coordinate.

\subsection{Propagation of the quantum field}

Now let us turn our attention to the quantum field and its propagation. We shall work with a minimally coupled massless scalar field $\phi$, so the field equation is

$$
\nabla_{a} \nabla^{a} \phi=0
$$

however the essentials of the argument would be the same for conformally coupled fields, or for fields of non-zero helicity.

The basic strategy is this. We assume the field in the distant past is specified. (For definiteness, we take it to be the vacuum, although the results would be the same for any reasonably quiescent state.) Thus we understand the expectations of combinations of the field operators $\phi(p)$ for events $p$ in the distant past. We therefore take the field operators in the distant past as initial data for the field equation. We may work out the field operators $\phi(q)$ for later events in terms of these data by solving the field equation, and then see what the quantum state looks like by forming expectations of these $\phi(q)$ 's. The expectations will be discussed in the next subsection; this subsection deals only with the problem of propagation.

Since the field is linear, it can be written as a sum (or integral) of c-number mode functions times ordinary creation and annihilation operators with no spacetime dependence. In other words, the operator character factors through the field equation, and we may discuss the propagation the field equation engenders without distinguishing between the mode functions and the corresponding operators.

Taking advantage of the spherical symmetry, we decompose the field into spherical

harmonics: $\phi=\sum_{l, m} \phi_{l, m} Y_{l, m}$. It is also convenient to extract a factor of $r$ from the 
field: we let $\phi=\phi^{0} / r$ and $\phi_{l, m}=\phi_{l, m}^{0} / r$. Then the fields $\phi_{l, m}^{0}$ satisfy reduced wave equations

$$
\partial_{u} \partial_{v} \phi_{l, m}^{0}+\quad \text { lower-order terms }=0
$$

where the lower-order terms contain a potential in the vacuum region and more complicated, time-dependent, terms in the matter region.

An explicit solution of these equations would clearly depend on the details of the metric within the matter, and thus we cannot reasonably expect to obtain this in generality. However, if we restrict our attention to those modes relevant to the detection of the field at late retarded times and in moderate frequency regimes, we can get an exact asymptotically valid formula, as first realized by Hawking.

Consider the observation of some field modes of moderate wavelengths $\sim \lambda_{\text {char }}$ at late retarded times and large spatial distances, in other words, for large $u$ and near $\mathcal{I}^{+}$. Here "moderate" means that we hold fixed a finite interval of wavelengths contributing to the wave-packet; doing so, we will find certain asymptotic behavior as $u \rightarrow+\infty, r \rightarrow+\infty .^{5}$ The important assumption is that the interval of wavelengths is not allowed to grow with $u$ or $r{ }^{6}$ In fact, the wavelengths of interest will turn out to be those of order $\sim R_{\text {Sch. }}$.

Let us consider the propagation of these field modes backwards in time in two stages, the first of which is unaffected by the matter or its collapse, and the second of which brings in the effects of the collapse on propagation. It is helpful to think of propagating the data from a Cauchy surface (that is, an initial-data surface) $\Sigma^{+}$where it is given, back in time to an intermediate Cauchy surface $\Sigma$ dividing the two stages, and finally back further to $\Sigma^{-}$(which will essentially be $\mathcal{I}^{-}$). Thus the propagation of the data from $\Sigma^{+}$to $\Sigma$ will involve only the exterior vacuum Schwarzschild geometry, whereas the propagation from $\Sigma$ to $\Sigma^{-}$will show the effects of the collapse.

\footnotetext{
${ }^{5}$ Strictly speaking, one cannot simultaneously have the wave packet bounded in $u$ and have its Fourier transforms bounded in wavenumber space. However one can arrange for either one to be bounded and the other to decay exponentially rapidly, or both to decay rapidly.

6 The important restriction here is actually the infrared one. To analyze infrared effects one would need to refine the analysis given here.
} 

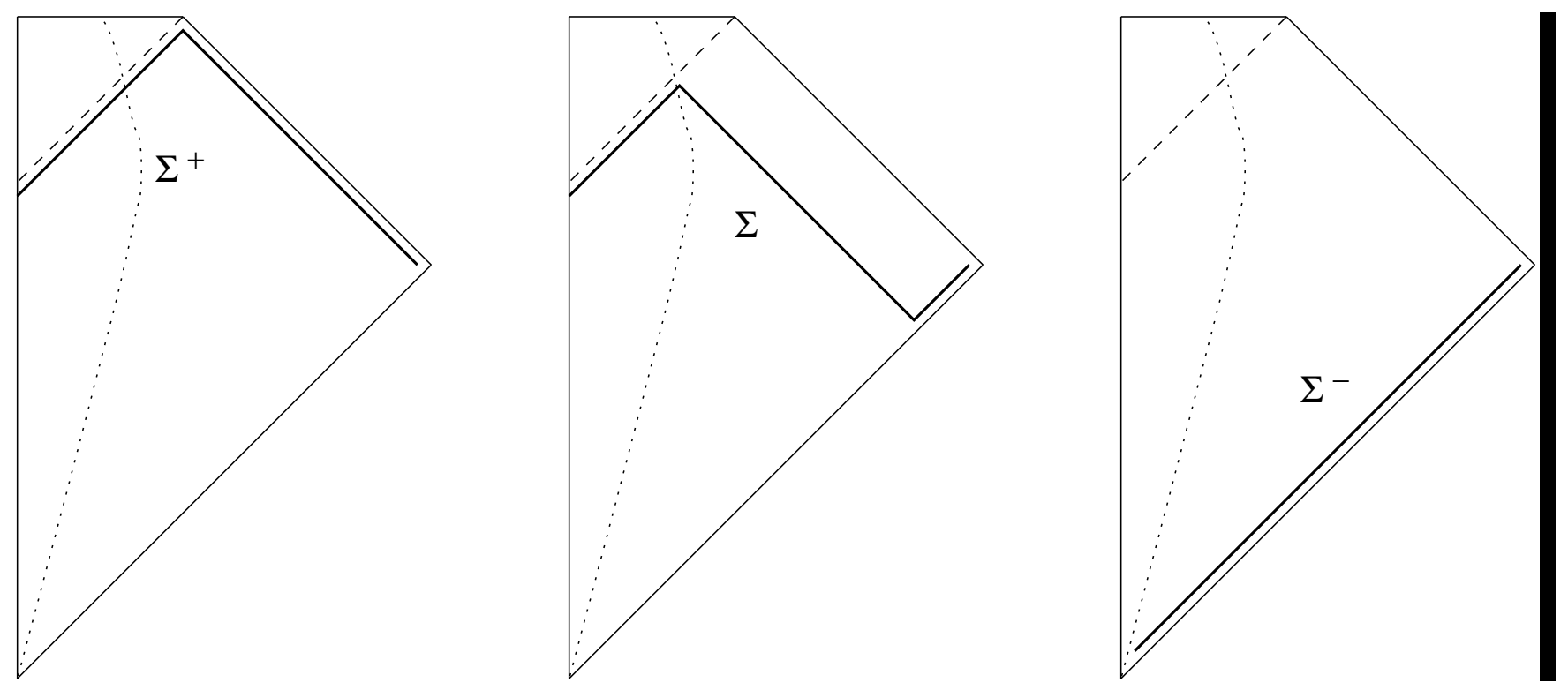

Figure 2. The three Cauchy surfaces used in propagating the field modes backwards in time from $\mathcal{I}^{+}$to $\mathcal{I}^{-}$. The portions in the black hole (on and above the event horizon) are given for conceptual completeness and do not contribute to the calculation.

In what follows, it will be convenient to refer to figure 2 . The field data we are given at late times on $\mathcal{I}^{+}$. While $\mathcal{I}^{+}$does not by itself constitute a complete Cauchy surface (roughly speaking, it contains no data for modes propagating into the hole), we may take $\Sigma^{+}=\mathcal{I}^{+} \cup \mathcal{H}^{+}$as a Cauchy surface on which our data are given (and also one could give data for field modes propagating into the hole). ${ }^{7}$ The inclusion of $\mathcal{H}^{+}$in the definition of $\Sigma^{+}$is really only for completeness - a Cauchy surface must be large enough to accommodate all possible data for the field. The data we are considering are identically zero on $\mathcal{H}^{+}$, because we are at present interested in what distant observers perceive, rather than in what happens at the event horizon.

As mentioned above, the precise definition of the Cauchy surfaces in the black-hole interior will not be important. It will be convenient to define $\Sigma$ in a sort of zig-zag, as follows. The boundary of the collapsing matter crosses the event horizon at some advanced time $v_{1}$. Let $\Sigma$ consist of three parts: the portion of the event horizon to the past of $v=v_{1}$; the portion of $v=v_{1}$ in the exterior of the hole; and the portion of $\mathcal{I}^{-}$to the future of $v=v_{1}$. By the causality of propagation, any data of the sort we are interested in, which were initially supported on $\mathcal{I}^{+}$, will give rise to non-zero data only on that portion of $\Sigma$ external to the hole. Indeed, the propagation of such signals from $\mathcal{I}^{+}$to $\Sigma$ depends only on a portion of the exterior vacuum Schwarzschild geometry.

The precise propagation is determined by the reduced wave equation governing the $\phi_{l, m}^{0}$ 's. It has the form (in the vacuum region)

$$
\partial_{u} \partial_{v} \phi_{l, m}^{0}+V_{l}(r) \phi_{l, m}^{0}=0,
$$

7 This is a bit of a simplification, because $\mathcal{H}^{+}$does not actually meet $\mathcal{I}^{+}$. We should more properly use a radially-symmetric null hypersurface slightly to the past of $\mathcal{H}^{+}$. There is a similar potential technicality involving the identification of $\Sigma^{-}$with $\mathcal{I}^{-}$. These issues will not be important here. 
where $V_{l}$ is a positive potential falling to zero as $r \rightarrow 2 M$ and $r \rightarrow+\infty$, and including a centrifugal term. As the wave packet propagates backwards from $\mathcal{I}^{+}$to $\Sigma$, it is partly dispersed and partly reflected by the potential. The reflection is a time-symmetric process (since reflected modes never enter the time-dependent region), and thus does not contribute directly to the Hawking process; it will be ignored. The dispersion means that the portion of the wave-packet that does propagate through the potential will arrive at $v=v_{1}$ somewhat distorted, and with a tail (falling off by a power law).

Now we come to a very important point. A given wave packet with wave profile $\left.\phi_{l, m}^{0}(u)\right|_{\mathcal{I}^{+}}$at $\mathcal{I}^{+}$will give rise to some profile $\left.\phi_{l, m}^{0}(u)\right|_{v=v_{1}}$ at $v=v_{1}$. We are interested in asymptotics for late times: the effect of translating by a time $T$ is to replace $u$ by $u+T$ in both of these profiles. Now recall that $u$ is not a good coordinate near the event horizon, but rather $v(u) \simeq v_{0}-C \exp -\kappa u$ is a good coordinate (cf. section 3.3). This means that the profile $\left.\phi_{l, m}^{0}(u)\right|_{v=v_{1}}$ is, as $T \rightarrow+\infty$, squeezed into an exponentially small portion of the $v=v_{1}$ surface, just before the horizon. In other words, in terms of any fixed local frame in the neighborhood of the point where the matter crosses the horizon, the relevant wave profiles become compressed into very tiny intervals; correspondingly, the frequencies of their components become exponentially blue-shifted.

We may thus, when we follow the waves back in time from $\Sigma$, apply the geometricoptics approximation. This approximation remains mathematically valid throughout this second stage of propagation, because there is nothing in this stage to undo the divergent blue-shift. The waves here propagate backwards in time, inwards through the matter, through the spatial origin, outwards through the matter again, and finally out to $\Sigma^{-}=\mathcal{I}^{-}$, but this portion of the trip only involves bounded red-shifts. Thus asymptotically (as $T \rightarrow+\infty$ ) the geometric-optics approximation becomes exactly valid for this second portion of the trip. One has simply

$$
\left.\phi_{l, m}^{0}(v(u))\right|_{\mathcal{I}^{-}}=\left.(-1)^{l+1} \phi_{l, m}^{0}(u)\right|_{v=v_{1}},
$$

where the factor $(-1)^{l+1}$ is due to reflection through the origin.

Notice that this result is universal (for the class of isolated spherically symmetric black holes of a given mass); it is independent of the details of the formation of the hole. It is undoubtably a very beautiful picture.

In order to compute the specifics of the field propagation, then, it only remains to work out $\left.\phi_{l, m}^{0}\right|_{v=v_{1}}$ from $\left.\phi_{l, m}^{0}\right|_{\mathcal{I}^{+}}$, a problem in vacuum Schwarzschild geometry. The result would be of the form

$$
\left.\phi_{l, m}^{0}(u)\right|_{\mathcal{I}^{+}}=\left.\int_{-\infty}^{u} K_{l}\left(u-u^{\prime}\right) \phi_{l, m}^{0}\left(u^{\prime}\right)\right|_{v=v_{1}} \mathrm{~d} u^{\prime},
$$

where $K_{l}$ is a suitable Green's function. (This is more commonly expressed in Fouriertransformed terms; here $K_{l}$ is essentially the Fourier transform of the transmission coefficients.) We shall not need the precise forms of the $K_{l}$ 's here. A very rough understanding of the propagation will be adequate for our needs. 
It is evident from the foregoing that the only scale which enters the wave propagation is set by the Schwarzschild radius. Thus it is only to be expected that the wavelengths predicted for the Hawking quanta must be of the order of this radius. As these are propagated backwards from $\mathcal{I}^{+}$, the modes with $l \geq 1$ are almost entirely reflected from the potential barrier. This reflection occurs entirely in the vacuum Schwarzschild region, which is time-symmetric, and so contributes little to the Hawking process. The Hawking process is therefore almost entirely an $\mathrm{s}-$ wave process.

Since the wavelengths involved are of the same scale as the Schwarzschild radius, one may expect that comparable fractions of the s-wave are reflected off the potential barrier and penetrate it. Similarly, the kernel $K_{0}$ must fall off beyond a length scale of the order of the Schwarzschild radius. It must have a $\delta\left(u-u^{\prime}\right)$ contribution (because of the $\partial_{u} \partial_{v}$ term in equation (19)), but apart from this it cannot have structure on scales much smaller than the Schwarzschild radius.

It is therefore not surprising that one can get a zeroth-order (order-of-magnitude) approximation to the physics by simply taking $K_{0}=\delta\left(u-u^{\prime}\right)$. This is the same as using the geometric-optics approximation for the propagation from $\Sigma^{+}$to $\Sigma$. (We emphasize that the geometric-optics model of propagation from $\Sigma$ back to $\Sigma^{-}$is expected to become rapidly asymptotically exact at late times. It is using geometric optics to model the propagation from $\Sigma^{+}$to $\Sigma$ which is a rough-and-ready approximation.) Then one has simply

$$
\left.\phi_{0,0}^{0}(u)\right|_{\mathcal{I}^{+}}=-\left.\phi_{0,0}^{0}(v(u))\right|_{\mathcal{I}^{-}}
$$

for the propagation of the fields, that is, the scattering. A more precise computation, including the kernel $K_{0}$, would involve only a small, exponentially compressed, smearing of the right-hand side.

The very simple scattering formula $(22)$ is the same as that for a field on one side of a moving mirror (perfect reflector) in two-dimensional Minkowski space-time, with $v=v(u)$ the trajectory of the mirror (and $u=t-x, v=t+x$ Minkowski null coordinates). In fact, moving mirrors were extensively investigated after Hawking's work, largely with the aim of clarifying aspects of it. We shall return to this point later.

To summarize, then. The propagation of the quantum fields is given very nearly exactly by (20) and (21), and to zeroth order (that is, accurately incorporating the main physical ideas but ignoring factors of order unity) simply by (19), where $v(u)$ is the mapping of surfaces of constant phase. We have the asymptotic behavior

$$
v(u) \simeq v_{0}-C \exp -\kappa u
$$

as $u \rightarrow+\infty$, that is, as the horizon is approached.

Note that the formula (23) depends on the black hole forming at a finite time (precisely, at the advanced time $v_{0}$ ). This assumption is used in propagating the field 
backwards in time out from the vicinity of the collapsing object and towards $\mathcal{I}^{-}$. For a hole formed at the very origin of the Universe, such an analysis would not apply, and one would have to consider the propagation of the field back in the vicinity of the initial singularity (the big bang). The treatment of such holes will not be considered here.

In Hawking's model, the behavior of the field for a range of $u$-values $\Delta u$ around $u$ will arise from initial field data from an exponentially compressed range of $v$-values, with $\Delta v \simeq v^{\prime}(u) \Delta u$ and $v^{\prime}(u) \simeq \exp -\kappa u$. This dependence of the predictions on the initial field values on exponentially fine scales is the trans-Planckian problem. It means that the Hawking quanta arise from the structure of the initial state at exponentially increasing frequencies and wave-numbers.

\subsection{The quantum state and the two-point functions}

We have so far discussed the evolution of the field operators; we now turn to the quantum state on which they act. This is specified as being a reasonably quiescent state in the distant past. We shall see in a little while that the precise choice is not too important; for simplicity, we shall take the state to be the in-vacuum, $\left|0_{\text {in }}\right\rangle$.

While it is natural to ask about the particle content of the state, it is for many purposes better to concentrate on the $n$-point functions, especially the two-point function, $\left\langle 0_{\text {in }}|\phi(p) \phi(q)| 0_{\text {in }}\right\rangle$. This is because the definition of "particle" is ambiguous here, as indeed it is in any problem where there is a non-stationary external potential (here, the time-dependent, collapse, phase of the space-time). Particles are defined in terms of creation and annihilation operators, which are in turn defined by splitting the field into positive- and negative-frequency components. When the field propagates through a non-stationary regime, this splitting is not preserved. Thus while the particle content of the theory is interesting, its analysis involves an extra issue, the splitting by frequencies, which is often best dealt with separately.

In fact, our primary interest is in whether the mathematical quantities appearing in Hawking's analysis (especially the field at very fine scales) can be regarded as credible representations of the real physics. This does not depend on whether one works in the particle representation or the field representation, and so analyzing the particle content of the theory is not really necessary for us. A brief sketch of this will be given, however, for completeness, in section 3.5.3.

We begin, then, by considering the two-point functions.

\subsubsection{The two-point function in Minkowski space.}

The state is supposed to be the in-vacuum. Since space-time is asymptotically flat, this means that near $\mathcal{I}^{-}$the two-point function has its Minkowskian form. So we shall begin by reviewing the two-point function of the massless scalar field in Minkowski space. This two-point function is

$$
\left\langle 0_{\mathrm{M}}|\phi(p) \phi(q)| 0_{\mathrm{M}}\right\rangle=-\frac{1}{4 \pi^{2}} \cdot \frac{1}{(p-q-\mathrm{i} \epsilon)^{2}},
$$


where $\epsilon$ is an infinitesimal future-directed timelike vector. Of course, strictly speaking it is not an ordinary function, but a distribution, and it must be averaged against test functions to be given meaning.

We can see a great deal of the physical content of the two-point function, and the part averages play, by considering a simple computation of vacuum fluctuations. Suppose we consider the average

$$
\Phi(a)=\left(4 \pi a^{3} / 3\right)^{-1} \int_{x^{2}+y^{2}+z^{2} \leq a^{2}} \phi(0, x, y, z) \mathrm{d} x \mathrm{~d} y \mathrm{~d} z,
$$

of a field operator over a sphere of radius $a$. The expected squared fluctuation in this is

$$
\begin{aligned}
\left\langle 0_{\mathrm{M}}|\Phi(a) \Phi(a)| 0_{\mathrm{M}}\right\rangle & =\left(4 \pi a^{3} / 3\right)^{-2}\left(4 \pi^{2}\right)^{-1} \int_{|\mathbf{x}| \leq a} \mathrm{~d}^{3} \mathbf{x} \int_{|\mathbf{y}| \leq a} \mathrm{~d}^{3} \mathbf{y}|\mathbf{x}-\mathbf{y}|^{-2} \\
& =\text { const } a^{-2}
\end{aligned}
$$

on dimensional grounds. (One can check that the integral is convergent.) Thus attempts to measure the field on a scale of size $a$ (equivalently, using modes with wavenumbers up to $\sim a^{-1}$ ) result in fluctuations of the order of $a^{-1}$. These are precisely the vacuum fluctuations. That these diverge as $a \rightarrow 0$ shows that questions of localizing fields are very delicate ones. By differentiating (24), and then integrating, one can also find fluctuations in the derivatives of the fields.

While the very simple form of (24) is a consequence of both the choice of state (vacuum) and the field equation (free massless in Minkowski space), its asymptotic behavior as $p \rightarrow q$ is expected to hold much more generally. Essentially, this is because the asymptotic regime $p \rightarrow q$ corresponds to ultra-high energies. For any fixed, "reasonable," state, near any event, there should be an energy scale beyond which the field modes are essentially unexcited, and also much higher than any masses appearing in the theory. In this case, one would expect the two-point function to approximate that of a massless field in vacuo. Such states are called Hadamard, and are generally regarded as the candidates for physically realistic states. ${ }^{8}$

Thus one expects asymptotic behavior like that of (24) as $p \rightarrow q$ to hold even in curved space-time, as the averaging scale $a \rightarrow 0$. We shall discuss the Hawking process in detail, below, but for the moment let us recall that we saw above that the Hawking quanta are supposed to arise from initial field data averaged over scales $a \sim R_{\text {Sch }} \exp -\kappa u$. This means that the Hawking quanta are supposed to arise from exponentially extreme field fluctuations at exponentially tiny scales in the in-state.

To connect these results with the calculations in the Hawking model, we decompose the two-point function into spherical harmonics. I shall only give the result for $l=0$ here, as that is the only one I shall use explicitly. This is got by averaging $p$ and

8 The discussion in this paragraph is a bit of a simplification, because the energy of a quantum field propagating on a background cannot be analyzed simply in terms of the frequencies of the modes. A more accurate statement would be that the Hadamard condition is preserved by propagation. 
$q$ over their spheres. Remembering that a factor of $r$ is absorbed in the expansion $\phi=r^{-1} \sum_{l, m} \phi_{l, m}^{0} Y_{l, m}$, one finds

$$
\begin{aligned}
& \left\langle 0_{\mathrm{M}}\left|\phi_{0,0}^{0}\left(u_{1}, v_{1}\right) \phi_{0,0}^{0}\left(u_{2}, v_{2}\right)\right| 0_{\mathrm{M}}\right\rangle \\
& \quad=-r_{1} r_{2}(4 \pi)^{-1} \int \sin \theta_{1} \mathrm{~d} \theta_{1} \mathrm{~d} \varphi_{1} \int \sin \theta_{2} \mathrm{~d} \theta_{2} \mathrm{~d} \varphi_{2}\left(\left(t_{1}-t_{2}-\mathrm{i} \epsilon\right)^{2}-\left(\mathbf{r}_{1}-\mathbf{r}_{2}\right)^{2}\right)^{-1} \\
& \quad=-\left(16 \pi^{2}\right)^{-1} \log \frac{\left(u_{1}-u_{2}-\mathrm{i} \epsilon\right)\left(v_{1}-v_{2}-\mathrm{i} \epsilon\right)}{\left(v_{1}-u_{2}-\mathrm{i} \epsilon\right)\left(u_{1}-v_{2}-\mathrm{i} \epsilon\right)}
\end{aligned}
$$

where $u=t-r$ and $v=t+r$. This is then the two-point function of a dimensionallyreduced field theory, a field which is a function of the coordinates $u$ and $v$ alone.

This $l=0$ two-point function can be understood in a very convenient way by thinking of the field in terms of its initial datum, that is, its value at $\mathcal{I}^{-}$. To see this, recall the classical result that for the $l=0$ solutions of the wave equation, the geometric-optics approximation is exact. (That is, any $l=0$ solution has the form $(f(u)+g(v)) / r$. $)$ When we require regularity of such solutions at the spatial origin, the solution is forced to depend on one function only. (The spatial origin is $u=v$; regularity there implies $f(u)=-g(v)$. One can consider $g(v)$ as the initial datum for the field..$^{9}$ ) Thus we may write

$$
\phi_{0,0}^{0}(u, v)=\left.\phi_{0,0}^{0}\right|_{\mathcal{I}^{-}}(v)-\left.\phi_{0,0}^{0}\right|_{\mathcal{I}_{-}}(u) .
$$

Comparing this with (27), we see that we can think of the initial datum for the field as a quantum field on $\mathcal{I}^{-}$with two-point function

$$
\left\langle\left. 0_{\mathrm{M}}\left|\phi_{0,0}^{0}\right|_{\mathcal{I}^{-}}\left(v_{1}\right) \phi_{0,0}^{0}\right|_{\mathcal{I}^{-}}\left(v_{2}\right) \mid 0_{\mathrm{M}}\right\rangle=-\left(16 \pi^{2}\right)^{-1} \log \left(v_{1}-v_{2}-\mathrm{i} \epsilon\right) .
$$

This logarithmic behavior is typical of quantum fields in two dimensions. The four factors in the logarithm in (27) arise from the different possible coincidences as the ingoing and outgoing radial null surfaces from $\left(u_{1}, v_{1}\right),\left(u_{2}, v_{2}\right)$ are extended back to $\mathcal{I}^{-}$, either directly or after passing through the origin.

\subsubsection{The two-point function in the Hawking model.}

We may apply the foregoing results directly to the Hawking model, since the assumption there is that the in-vacuum has the Minkowskian form in the asymptotic past. If we use the zeroth-order approximation (only $l=0$ modes contribute to the Hawking process, and those propagate by geometric optics), then we have

$$
\begin{aligned}
& \left\langle 0_{\text {in }}\left|\phi_{0,0}^{0}\left(u_{1}, v_{1}\right) \phi_{0,0}^{0}\left(u_{2}, v_{2}\right)\right| 0_{\text {in }}\right\rangle \\
& \quad=\left\langle 0_{\text {in }}\left|\left(\left.\phi_{0,0}^{0}\right|_{\mathcal{I}^{-}}\left(v_{1}\right)-\left.\phi_{0,0}^{0}\right|_{\mathcal{I}^{-}}\left(v\left(u_{1}\right)\right)\right)\left(\left.\phi_{0,0}^{0}\right|_{\mathcal{I}^{-}}\left(v_{2}\right)-\left.\phi_{0,0}^{0}\right|_{\mathcal{I}^{-}}\left(v\left(u_{2}\right)\right)\right)\right| 0_{\text {in }}\right\rangle \\
& \quad=-\left(16 \pi^{2}\right)^{-1} \log \frac{\left(v\left(u_{1}\right)-v\left(u_{2}\right)-\mathrm{i} \epsilon\right)\left(v_{1}-v_{2}-\mathrm{i} \epsilon\right)}{\left(v\left(u_{1}\right)-v_{2}-\mathrm{i} \epsilon\right)\left(v_{1}-v\left(u_{2}\right)-\mathrm{i} \epsilon\right)} .
\end{aligned}
$$

${ }^{9}$ In the classical context, one is free to add a constant to $f$ and subtract the same constant from $g$, so there is an ambiguity in this choice of initial datum, which does not affect the field. There is a similar freedom in the quantum case, which will not be important here. 
Had we used the exact treatment, the only difference would be a mild smearing of the $v(u)$ terms, over ranges of size $\Delta u \lesssim R_{\mathrm{Sch}} / c$ (and the inclusion of similar formulas, but much smaller ones, for higher $l$ values).

We shall consider only local field measurements, so $\left(u_{1}, v_{1}\right),\left(u_{2}, v_{2}\right)$ are nearby. Then the two-point function is large. The important factors in the logarithm are those nearing zero, $\left(v\left(u_{1}\right)-v\left(u_{2}\right)-\mathrm{i} \epsilon\right)$ and $v_{1}-v_{2}-\mathrm{i} \epsilon$.

Of these, the second has the form it does in Minkowski space, and so represents the same vacuum fluctuations one would have there. The interesting effects are those which differ from the Minkowskian ones, that is, the renormalized two-point function

$$
\begin{aligned}
& \left\langle 0_{\text {in }}\left|\phi_{0,0}^{0}\left(u_{1}, v_{1}\right) \phi_{0,0}^{0}\left(u_{2}, v_{2}\right)\right| 0_{\text {in }}\right\rangle-\left\langle 0_{\mathrm{M}}\left|\phi_{0,0}^{0}\left(u_{1}, v_{1}\right) \phi_{0,0}^{0}\left(u_{2}, v_{2}\right)\right| 0_{\mathrm{M}}\right\rangle \\
& =-\left(16 \pi^{2}\right)^{-1} \log \frac{v\left(u_{1}\right)-v\left(u_{2}\right)-\mathrm{i} \epsilon}{u_{1}-u_{2}-\mathrm{i} \epsilon}+\text { less significant terms }
\end{aligned}
$$

For $\left|u_{1}-u_{2}\right| \ll R_{\text {Sch }}$, the function $v(u) \simeq v_{0}-C \exp -\kappa u$ may be approximated linearly, and we have

$$
v\left(u_{1}\right)-v\left(u_{2}\right) \simeq v^{\prime}(u)\left(u_{1}-u_{2}\right)
$$

Thus the divergent parts of the Hawking and Minkowski two-point functions cancel for $\left|u_{1}-u_{2}\right| \ll R_{\text {Sch}}$. This is the Hadamard condition discussed above, that on sufficiently fine scales the two-point function should approach the Minkowskian one.

If we consider points which are a bit more separated, say $\left|u_{1}-u_{2}\right| \sim R_{\text {Sch }}$, the nonlinearities of $v(u)$ become significant over the range of $u$ in question, and thus the Hawking analysis predicts significant excitations at these scales. This means that the production of quanta becomes significant for wavelengths $\sim R_{\text {Sch }}$; this is Hawking's prediction. The fact that the spectrum is thermal with temperature $T_{\mathrm{H}}=\hbar \kappa /(2 \pi c k)$ is essentially due to the fact that expressions like (31) are periodic in imaginary time with period $2 \pi c / \kappa$ (KMS condition). ${ }^{10}$ Because the zeroth-order approximation is reasonably good, the frequency-dependence of the transmission of the relevant modes through the space-time is weak, and the spectrum is close to a Planckian, black-body, one. Notice that the argument for thermality uses essentially the exponential form of $v(u)$ at late retarded times.

We can see here that the Hawking quanta, which correspond to disturbances near $\mathcal{I}^{+}$extended over $\Delta u \sim R_{\mathrm{Sch}}$, do indeed arise from vacuum fluctuations in the past over scales $\sim R_{\mathrm{Sch}} v^{\prime}(u) \sim R_{\mathrm{Sch}} C \exp -\kappa u$. This exponential compression

10 The Kubo-Martin-Schwinger (KMS) condition characterizes thermal states. If a system has a Hamiltonian $H$ and is in thermal equilibrium with inverse temperature $\beta$, then it will be described by a density matrix $\rho=\exp -\beta H$. For any two observables $A(t), B(t)$ (where the time-dependence is given by the Hamiltonian evolution), we should have formally $\left\langle A\left(t_{1}\right) B\left(t_{2}\right)\right\rangle=\operatorname{tr}(\exp -\beta H) A\left(t_{1}\right) B\left(t_{2}\right)=$ $\operatorname{tr} B\left(t_{2}\right)(\exp -\beta H) A\left(t_{1}\right)=\operatorname{tr} B\left(t_{2}\right) A\left(t_{1}+\mathrm{i} \beta\right)(\exp -\beta H)=\operatorname{tr}(\exp -\beta H) B\left(t_{2}\right) A\left(t_{1}+\mathrm{i} \beta\right)=\left\langle B\left(t_{2}\right) A\left(t_{1}+\right.\right.$ $\mathrm{i} \beta)\rangle$. The equality of the left-most and right-most terms is the KMS condition. It includes periodicity in imaginary time as as the special case $B=1$. So the argument given above for the Hawking process is not sufficient for establishing the KMS condition, but it is necessary. A full, careful, treatment reveals no surprises. 
means that (after a finite passage of retarded time), one is probing deeply enough the ultraviolet asymptotics of the in-state's two point function, that any such state which is Hadamard will produce the same result. In other words, it is not necessary to assume that the in-state is the vacuum, but only that it is Hadamard.

On the other hand, taking this unbounded exponential compression of the scales at face value may fairly be called fantastic. The energies of the in-modes in question very quickly surpass, not just the Planck energy, but the entire estimated energy of the Universe.

To emphasize the essential way these high-frequency modes enter, suppose we had initially imposed an ultraviolet cut-off $\Lambda$ on the in-modes. Then we should have found no Hawking quanta at late times, for the out-modes' maximum frequency would be $\sim v^{\prime}(u) \Lambda$, which goes to zero rapidly. (It is worth pointing out that this procedure is within what may be fairly described as text-book quantum field theory: start with a cut-off, do the calculation, and at the very end take the cut-off to infinity. That this results in no Hawking quanta emphasizes the delicacy of the issues. In this sense, the trans-Planckian problem may be thought of as a renormalization-ambiguity problem.)

\subsubsection{Particles.}

As mentioned earlier, our real concerns are with Hawking's use of field modes of exponentially increasing frequencies, not whether we express the physics of those modes in terms of particles or fields, two alternate quantum representations. However, for completeness we include a brief outline of the analysis in particle terms.

Particles are defined in terms of the creation and annihilation operators of the field, and those in turn are determined by splitting the field into negative- and positive-frequency parts. Suppose a physicist carries a device (like a photomultiplier) whose output he interprets as particle counts. That device functions according to its own internal physics (responding to whatever fields are around); in particular, it presumably uses its own local notion of proper time to distinguish positive from negative frequencies. Thus a device in the "in" region in the gravitational collapse problem will essentially be distinguishing positive from negative frequencies on the basis of Fourier transforms with respect to $v$ (near $\mathcal{I}^{-}$), whereas one in the "out" region will make the distinction based on Fourier transforms with respect to $u$ (near $\left.\mathcal{I}^{+}\right)$.

Precisely because the space-time is not stationary, a field mode which is $v$-positive frequency will propagate through the space-time into a mixture of $u$-positive and $u-$ negative frequencies. The in-vacuum is characterized as the state annihilated by all $v$-positive-frequency field operators. However, owing to the mixing, this will not be annihilated by all $u$-positive-frequency operators. It will not be the out-vacuum.

This is most commonly expressed in terms of Bogoliubov transformations. We write schematically

$$
\begin{aligned}
& \phi_{\text {out }}^{+}=\alpha \phi_{\text {in }}^{+}+\beta \phi_{\text {in }}^{-} \\
& \phi_{\text {out }}^{-}=\alpha^{*} \phi_{\text {in }}^{-}+\beta^{*} \phi_{\text {in }}^{+},
\end{aligned}
$$


where the mode indices have been suppressed. (So really $\alpha, \beta$ are infinite continuous or discrete matrices.) The $\alpha$ 's and the $\beta$ 's are the Bogoliubov coefficients. By using the commutation relations, one can show that the expected number of particles created is simply

$$
\operatorname{tr} \beta^{*} \beta \text {. }
$$

Since the analysis is mode-by-mode, the number of quanta in a given out-mode is simply the sum of $\beta^{*} \beta$ for this out mode (the matrix multiplication effecting the sum over all the in-modes).

In fact this was how Hawking did his analysis. One can find the $\alpha$ 's and $\beta$ 's by taking the positive- and negative-frequency parts of the equations for the propagation of the field from $\mathcal{I}^{-}$to $\mathcal{I}^{+}$, equations (20), (21). Then one can explicitly compute the expected spectrum as a function of frequency.

\subsection{Stress-Energy}

One would like to understand the energy-momentum budget of the Hawking process. This has not been fully achieved, on account of fundamental difficulties in constructing (Wald 1994) and interpreting (Helfer 1996, 1998) the stress-energy operator for quantum fields in curved space-time.

In order to get a finite stress-energy operator, one needs to renormalize, and there are ambiguities in how to do this. In Minkowski space, these are resolved by appealing to Poincaré invariance, but in curved space-time, in general circumstances, it is not known how to fix the "finite part" of the stress-energy operator (Wald 1994).

It should also be remarked that, while, up to the problem of getting the finite part right, the stress-energy operator is well-defined, the sense in which it is well-defined is that it becomes an operator-valued distribution. That is, in general it must be averaged against a tensor test-function over a space-time volume in order to really be a self-adjoint operator. If one tries to average against a tensor test function over, for example, a Cauchy surface, one does not in generic circumstances get a self-adjoint operator, but an apparently pathological object (Helfer 1996, 1998). These issues are not wholly understood and will not be discussed further here, however, they certainly raise questions about whether we have an adequate understanding of the energetics of the Hawking process.

In an asymptotically flat regime (for example, near $\mathcal{I}^{+}$), one can use the Minkowskian structure to identify the correct renormalization. This enables us to conclude that the stress-energy of the state predicted by Hawking, measured by distant observers, should indeed be that of a quasi-thermal state. The calculations involved even in this case are not trivial, for the renormalization must be done carefully. However, using the fact that our "zero-order" approximation is mathematically identical to a moving-mirror model, we can make use of general formulas in the moving-mirror case (Fulling and Davies 1976, Davies and Fulling 1977) to conclude 
that in this approximation

$$
\begin{aligned}
\left\langle T_{a b}\right\rangle & =\left(12 \pi r^{2}\right)^{-1} \hbar\left(\frac{3}{4}\left(\frac{\ddot{v}}{\dot{v}}\right)-\frac{1}{2} \frac{v^{(3)}}{\dot{v}}\right) l_{a} l_{b} \text { as } r \rightarrow \infty \\
& =\left(48 \pi r^{2}\right)^{-1} \hbar \kappa^{2} l_{a} l_{b} \quad \text { as } \quad r \rightarrow \infty
\end{aligned}
$$

where $l^{a}$ is the outgoing null vector. This is indeed the stress-energy of a radiative flux of energy; it is in fact the correct asymptotic form up to a numerical factor (attributable to the neglect of higher-angular momentum modes and transmission coefficients). But this is only what one would expect. The interesting question is what the stress-energy is at finite values of $r$, and particularly near the horizon.

While, as explained above, it is not known precisely how to compute the stressenergy at finite values of $r$, there are two important statements about it one can make. The first is that the only difficulties associated with renormalizing it are in fixing the finite part and the interpretational issues raised earlier. Thus (unless somehow the correct choice of c-number becomes divergent at the horizon - something which could not happen if the choice is fixed by local physics), the stress-energy must be as regular at the horizon as it is anywhere else. The second is that given any reasonable, locally conserved, candidate definition for the effective stress-energy which is stationary at late times, the flux of energy outwards across $\mathcal{I}^{+}$must equal minus the flux across the event horizon. This means that the energy flux into the black hole is negative.

To see this, let us suppose that there exists a well-defined classical effective stressenergy $T_{a b}^{\mathrm{eff}}$. We shall not be concerned with precisely how this is computed; the argument will be independent of that. We shall however require the tensor to be conserved, regular (of class $C^{1}$ ), and stationary in the quasistationary regime under consideration, including the future horizon. Let $\Sigma$ be a hypersurface in this regime from a cut $S_{\mathcal{H}^{+}}$of the event horizon to a cut $S_{\mathcal{I}^{+}}$. We may compute the effective energy on $\Sigma$,

$$
\int_{\Sigma} T_{a b}^{\mathrm{eff}} \xi^{a} \mathrm{~d} \Sigma^{b}
$$

where $\xi^{a}=\partial / \partial t$ is the Schwarzschild Killing vector. Now imagine flowing $\Sigma$ forward along $\xi^{a}$, to some $\Sigma_{t}$. The energies on $\Sigma$ and on $\Sigma_{t}$ will be identical, because $T_{a b}^{\mathrm{eff}}$ and the Schwarzschild geometry are stationary. However, since $T_{a b}^{\mathrm{eff}}$ is conserved, this means that the energy flux across the portion of $\mathcal{I}^{+}$from $S_{\mathcal{I}^{+}}$to its image $S_{\mathcal{I}^{+}}^{t}$ under the flow must be compensated by a negative flux across the horizon from $S_{\mathcal{H}^{+}}$to its image $S_{\mathcal{H}^{+}}^{t}$.

This is just what one would expect on the basis of the Area Theorem: if Hawking radiation carries energy away from the hole, the hole will lose mass, hence area, and this must mean a negative energy flux is crossing the horizon.

The picture that one has, then, is that the in-state (vacuum) is being split by its passage through the collapsing geometry into positive- and negative-energy fluxes. The negative-energy fluxes fall into the black hole, while the positive-energy ones escape to infinity. 


\subsection{The analysis of Fredenhagen and Haag}

Hawking's original analysis was in terms of Bogoliubov coefficients, with the modes taken to be Fourier ones. An alternative computation based more directly on the twopoint functions was given by Fredenhagen and Haag (1990). Their results reproduced Hawking's, but clarified certain issues.

First, because the singularities of the two-point functions are local, Fredenhagen and Haag were able to bring out very clearly the dependence of the computation on the Hadamard form of the initial state and (in the notation of section 3.4) its propagation between the surfaces $\Sigma$ and $\Sigma^{+} .{ }^{11}$ Fredenhagen and Haag emphasized that, given the Hadamard form of the two-point function on $\Sigma$, one would reproduce Hawking's results. This seems to have been misinterpreted by some workers as meaning that the analysis resolved the trans-Planckian problem. However, this is not the case. The Fredenhagen-Haag analysis confirms Hawking's, and, as we shall see in detail in the next section, that propagation involves trans-Planckian problems.

Second, their analysis gave better control of infrared issues than did Hawking's. Fredenhagen and Haag were able to show that the Hawking process is asymptotically stationary. This is plausible but not quite clear in Hawking's analysis. This is because mild infrared divergences (which are hard to rule out in Hawking's approach) could give rise to secular growth in the $n$-point functions. That this does not occur requires rather careful estimates, which are outlined in Fredenhagen and Haag's paper.

\subsection{Almost-black holes}

What happens if a gravitating object collapses to the verge of forming a black hole, but never quite does so? Suppose, say, a star collapses to $R=(1+\epsilon) R_{\text {Sch }}$ but then becomes stationary? It is useful to examine this, both to deepen our understanding of Hawking's model and to be able to compare its predictions with those of others.

In the Hawking model, while some transient quanta may be formed, after a while (perhaps a long while), the modes detected by distant observers arise almost entirely by propagation through a stationary region, and so there are no Hawking quanta.

The interval of transience is determined as follows. Consider the usual argument about tracing modes backwards in time through the collapsing object. The mode encounters the limb of the object first at a radius which for late times will be $(1+\epsilon) R_{\mathrm{Sch}}$. It propagates backwards in time through the object, emerging at a radius say $r$. The question is whether $r$ is equal to $(1+\epsilon) R_{\mathrm{Sch}}$ or greater than it. At sufficiently late times, this part of the limb has contracted to $(1+\epsilon) R_{\text {Sch }}$ and (by hypothesis) goes no further. This means the propagation of the mode inwards to the object and outwards from the object are symmetric, there is no red-shift and there are no Hawking quanta. On the other hand, in the transient regime, the radius $r$ is still somewhat larger than $(1+\epsilon) R_{\mathrm{Sch}}$, and thus the blue-shift of the mode in from $\mathcal{I}^{+}$to $(1+\epsilon) R_{\mathrm{Sch}}$ is larger than the red-shift out from $r$ to $\mathcal{I}^{-}$, and one has the possibility of production of quanta.

11 Actually, Fredenhagen and Haag did not use precisely our surface $\Sigma$, but a similar one. 
In other words, the period of transience ends when a distant observer, looking through the collapsing object, sees its trailing edge reach $(1+\epsilon) R_{\text {Sch }}$. After this, no quanta are expected.

\section{The trans-Planckian problem}

We have seen that the quanta which are supposed to be produced by the Hawking process at late retarded times $u$, that is, the physically dominant modes of the quantum field, have their origins near $\mathcal{I}^{-}$in vacuum fluctuations of frequencies $\sim\left(c / R_{\mathrm{Sch}}\right)\left(v^{\prime}(u)\right)^{-1}$, where

$$
v(u) \simeq v_{0}-C \exp -\kappa u .
$$

We also saw that the exponential form of this mapping of surfaces of constant phase was integral to the thermal character of the final state.

Exponential relations like (37) are never accepted uncritically in physics. They are never supposed to hold for arbitrarily long times. There always comes a point, after some number of e-foldings, when one has passed the scales at which the mathematical models used are valid, and new physical effects must be considered.

In our case, the frequencies of the original modes quickly pass (not only all conventional quantum field-theoretic scales, but) the Planck scale. This is called the trans-Planckian problem. It is clear that any analysis that relies on assumptions about physics at the Planck scale is speculative, and that an analysis which relies on the application of conventional physics beyond the Planck scale is questionable.

The foregoing comments are negative ones, and one would like to approach the trans-Planckian issue constructively. In order to do this, we must try to be as precise as possible about how the trans-Planckian modes are bound up with the physics of the collapse.

The main aim of this section is to show that the trans-Planckian problem can be localized to two regimes. Roughly speaking, these are a neighborhood of the event horizon, and a neighborhood of the surface of advanced time $v=v_{0}$ at which the event horizon forms. This means that if somehow one could find alternative, cis-Planckian, physics to that of the Hawking model which operated in those regimes, but reproduced Hawking's results elsewhere, one would have overcome the trans-Planckian problem. This will be discussed in section 8 .

Strictly speaking, we cannot expect a localization of the trans-Planckian problem in space-time, but must pass to the frame bundle. This is because frequency is a frame-dependent quantity, and so the trans-Planckian regime really consists of all those frames, at different events, for which the characteristic field modes giving rise to Hawking quanta have trans-Planckian frequencies. This will be analyzed carefully below, and it will be shown that for certain important cases the trans-Planckian regime extends substantially away from the event horizon. These have implications for models where black holes are formed by sending in massless particles or dust. 
The most important frames, physically, are those defined by the matter whose collapse drives the formation of the black hole. (At any given event in the matter, there may be a family of natural frames. These will all differ from each other by bounded boosts.) Field modes which give rise to Hawking quanta and become transPlanckian in such frames are the problematic ones. Knowledge of precisely what happens to these modes requires some knowledge of Planck-scale physics, which presumably means quantum gravity. In some sense, one would expect the modes to become entangled with quantum-gravitational fluctuations in the collapsing matter. The Hawking model, which relies on "painting" the field modes on a fixed classical space-time, would not be valid.

As mentioned above, there are two trans-Planckian regimes: one near the event horizon; and the other near the surface of constant advanced time $v=v_{0}$ at which the event horizon forms. Only the former will require detailed analysis here. The latter is equally important, but for our purposes it will be enough to note that the frequencies of the field modes diverge there with respect to the asymptotic rest frame.

\subsection{Localization of the problem}

Here we study how the trans-Planckian problem may be localized. We will need to take into account the fact that frequency is not a scalar, but an observer-dependent quantity, so that different observers will have different notions of when a given wavevector passes the Planck scale. Even a visible photon would, according to special relativity, appear to have Planck energy to sufficiently boosted observers. This means that a full treatment really localizes the trans-Planckian problem, not in space-time, but in the bundle of frames. The localization consists of those frames of observers for whom the field modes giving rise to Hawking quanta are trans-Planckian. For every event in space-time, there are some trans-Planckian frames, corresponding to sufficiently boosted observers.

However, not all mathematically constructible frames are relevant to the physics of the situation. In fact, we distinguish two main classes of frames of interest:

(CH) Those of observers whose world-lines cross the horizon;

(SO) Those of stationary observers (that is, observers moving along the timelike Killing field $\partial / \partial t$ ).

We will develop a general framework for analyzing the problem, and then specialize it to these cases.

We use the standard Schwarzschild coordinates, and put

$$
f^{2}=1-\frac{R_{\mathrm{Sch}}}{r} \text {. }
$$

Then an orthonormal frame (in the $(t, r)$ space) is given by

$$
T^{a}=f^{-1} \partial_{t}, \quad R^{a}=f \partial_{r} .
$$

It will also be convenient to introduce the associated null frame

$$
L^{a}=2^{-1 / 2}\left(T^{a}+R^{a}\right), \quad N^{a}=2^{-1 / 2}\left(T^{a}-R^{a}\right) .
$$


We have

$$
\begin{aligned}
L^{a} \nabla_{a} v & =L^{a} \nabla_{a}\left(t+r_{*}\right) \\
& =2^{1 / 2} f^{-1},
\end{aligned}
$$

and similarly for $N^{a} \nabla_{a} u$, so in $(u, v)$ coordinates we have

$$
L^{a}=2^{1 / 2} f^{-1} \partial_{v}, \quad N^{a}=2^{1 / 2} f^{-1} \partial_{u} .
$$

If an observer is boosted to a velocity $c \tanh \xi$ radially outward from this frame, her frame will be

$$
T_{\xi}^{a}=\cosh \xi T^{a}+\sinh \xi R^{a}, \quad R_{\xi}^{a}=\sinh \xi T^{a}+\cosh \xi R^{a},
$$

or

$$
L_{\xi}^{a}=e^{\xi} L^{a}, \quad N_{\xi}^{a}=e^{-\xi} N^{a} .
$$

The wave covector for a field mode producing a Hawking quantum (of characteristic wavenumber $R_{\mathrm{Sch}}^{-1}$ ) is

$$
K_{a}=R_{\mathrm{Sch}}^{-1} \mathrm{~d} u
$$

and the corresponding vector is

$$
K^{a}=2^{-1 / 2} f^{-1} R_{\mathrm{Sch}}^{-1} L^{a} .
$$

Thus the trans-Planckian problem occurs for

$$
\begin{aligned}
l_{\mathrm{Pl}}^{-1} & \lesssim g_{a b} T_{\xi}^{a} K^{b} \\
& =\mathrm{e}^{-\xi} f^{-1} R_{\mathrm{Sch}}^{-1},
\end{aligned}
$$

or equivalently

$$
\mathrm{e}^{\xi} f \lesssim l_{\mathrm{Pl}} / R_{\mathrm{Sch}}
$$

The formula just given is the frame-bundle form of the localization of the transPlanckian problem. Interestingly, it does not depend explicitly on the retarded time $u$, although for a given family of observers both $r$ (and hence $f=f(r)$ ) and $\xi$ may depend on $u$. Unless $r$ is close to $R_{\text {Sch }}$, the function $f$ is of order unity, and thus the trans-Planckian modes arise only for

$$
\mathrm{e}^{-\xi} \gtrsim R_{\mathrm{Sch}} / l_{\mathrm{Pl}}
$$

Thus, for observers not close to $r=R_{\text {Sch }}$, the trans-Planckian issue arises only for high inward boosts. For observers close to $r=R_{\mathrm{Sch}}$, it is important to discuss the physical definition of the frame in question before interpreting the formulas. Thus we examine our two families of observers. 


\subsubsection{The family $(\mathrm{CH})$.}

The family $(\mathrm{CH})$ of observers crossing the horizon is the most important one, because particular cases of it correspond to frames of the matter whose collapse drives the formation of the black hole. We shall find, rather remarkably, that the onset of the trans-Planckian problem occurs for such observers at a local time $\sim t_{\mathrm{Pl}}$ before they cross the horizon, independent of the velocity at which they cross. The implications of this will be discussed in section 4.2 .

We will specify the families of observers in the vacuum region; the behavior of observers tracking the limb of the collapsing object may be obtained from this. (We shall not consider the interior of the collapsing matter. What happens there must connect continuously with the case we analyze, and that will be a strong enough result for us.)

To parameterize the observers in a physically meaningful way, let us start from the standard null frame $L^{a}, N^{a}$ at $\mathcal{I}^{-}$, and parallel transport it along the inward direction $N^{a}$ to the event horizon. One finds that the inwardly-transported null frame is $f L^{a}$, $f^{-1} N^{a}$. If we consider a frame boosted outwards by $c \tanh \zeta$ from this, its unit timelike vector is

$$
2^{-1 / 2}\left(f \mathrm{e}^{\zeta} L^{a}+f^{-1} \mathrm{e}^{-\zeta} N^{a}\right) .
$$

The normalized null vectors in the frame are evidently $f \mathrm{e}^{\zeta} L^{a}, f^{-1} \mathrm{e}^{-\zeta} N^{a}$. One can interpret $c \tanh \zeta$ as a measure of the observer's velocity as it crosses the horizon. Larger positive values correspond to shallower crossings; more negative values correspond to sharper ones.

To connect with the notation of the previous subsection, we have $\mathrm{e}^{\xi}=f \mathrm{e}^{\zeta}$, and the condition for the characteristic field modes to appear trans-Planckian is (from (48))

$$
\mathrm{e}^{\xi} f=\mathrm{e}^{\zeta} f^{2} \lesssim l_{\mathrm{Pl}} / R_{\text {Sch }} .
$$

Thus for any fixed $\zeta$, the trans-Planckian regime consists of events with coordinate values of $r$ sufficiently close to $R_{\text {Sch }}$. For shallower crossings of the horizon (larger $\zeta$ ), the regime is smaller, whereas for sharper crossings it is larger.

To get a better understanding of this, let us ask how the function $f^{2}$ appears to depend on our observer's local time. We have

$$
\begin{aligned}
T_{\xi}^{a} \nabla_{a} f^{2} & =2^{-1 / 2}\left(T^{a} \cosh \xi+R^{a} \sinh \xi\right) \nabla_{a} f^{2} \\
& =2^{-1 / 2} \sinh \xi f \partial_{r} f^{2} \\
& =2^{-3 / 2}\left(f \mathrm{e}^{\zeta}-f^{-1} \mathrm{e}^{-\zeta}\right) f R_{\mathrm{Sch}} / r^{2} \\
& \simeq-2^{-3 / 2} \mathrm{e}^{-\zeta} R_{\mathrm{Sch}}^{-1}
\end{aligned}
$$

near the horizon. This means that if we choose the zero of the local time $t_{\text {loc }}$ to be when the observer crosses the horizon, we have

$$
f^{2} \simeq-2^{-3 / 2} \mathrm{e}^{-\zeta} R_{\mathrm{Sch}}^{-1} t_{\mathrm{loc}} .
$$


Using this, we may recast the condition (51) for the characteristic modes to become trans-Planckian as

$$
\mathrm{e}^{\zeta} f^{2} \simeq 2^{-3 / 2} R_{\mathrm{Sch}}^{-1}\left|t_{\mathrm{loc}}\right| \lesssim l_{\mathrm{Pl}} R_{\mathrm{Sch}}^{-1}
$$

which is simply (dropping the $2^{-3 / 2}$ )

$$
\left|t_{\mathrm{loc}}\right| \lesssim t_{\mathrm{Pl}}
$$

This result is, curiously, independent of $\zeta$.

In other words, for any observer whose world-line crosses the event horizon, the onset of the trans-Planckian regime will be at a local time $\sim t_{\mathrm{Pl}}$ before the crossing. This result does not depend on whether the observer is freely falling or not; it depends only on the world-line being of class $C^{1}$.

The case of observers crossing the horizon ultrarelativistically inwards is of special interest. For these observers, the increment of the world-line of length $t_{\mathrm{Pl}}$ before the event horizon extends far from the horizon. In particular, if we were to consider an ultrarelativistic collapse of matter to form the black hole, then the onset of the transPlanckian problem could occur well before the event horizon (in the frames of observers who are not ultrarelativistically boosted). This issue should be kept in mind when evaluating models where black holes are formed by ultrarelativistic (or null) collapse.

\subsubsection{The family (SO).}

We consider here the family of stationary observers. For these, the timelike unit tangent is simply $T^{a}$, and so this is the case $\xi=0$. We find that the trans-Planckian problem is manifest for

$$
f \lesssim l_{\mathrm{Pl}} / R_{\mathrm{Sch}}
$$

This regime is properly contained in that for the horizon-crossing observers, since $f^{2}<f$. Since the proper radial distance of an event near the horizon to the horizon is $\simeq 2 R_{\mathrm{Sch}} f$, another way of expressing (56) is to say that the proper radial distance of the observer from the horizon should be $\lesssim l_{\mathrm{Pl}}$.

It turns out that the trans-Planckian problem for the Hawking modes for stationary observers manifests itself at the same scale as another difficulty, the point where the accelerations of the observers become so great they cannot be considered meaningful classically. This latter occurs when the local acceleration experienced by the observer becomes of the order $l_{\mathrm{Pl}} / t_{\mathrm{Pl}}^{2}$. (One can easily check this by computing the local acceleration; it turns out to be $G M f^{-1} r^{-2}$.)

\subsection{Discussion}

We have seen that the trans-Planckian problem is essentially a local one, except for the case of ultrarelativistic collapse. This means that if one could somehow get the modes through these problematic regions by alternative, cis-Planckian, one would have a solution to the trans-Planckian problem. 
Discussions of this are sometimes phrased in terms of "getting the right vacuum" outside of the event horizon. This is picturesque but can be misleading. What is really meant is getting the state, and in particular, the $n$-point functions, right. However, we may accurately say that the problem is to understand how to propagate the in-vacuum to the neighborhood of the event horizon.

\section{Connection with the Unruh process}

One might hope that, given the very beautiful and simple form of Hawking's predictions, they could be recovered from alternative physical arguments. In particular, if the existence of Hawking radiation really did not depend on ultra-high energy physics or quantum-gravitational hypotheses, one would hope for alternative arguments within the realm of conventional physics.

Despite an enormous amount of work on the Hawking process, no such arguments exist, and indeed very little of the work has confronted this problem directly. The aim of most research has been not so much to address the foundational difficulties of Hawking's analysis as to show that there are connections between that analysis and known physics. In other words, most of the work really simply assumes Hawking's analysis is correct, and, on that basis, looks for connections with other physics. Such work may nevertheless lead to circumstantial evidence for the Hawking effect, as will be discussed in section 7 .

In one sense, it is clear that it will be impossible to dodge the trans-Planckian problem, or the issue of quantum-gravitational hypotheses, and present a thoroughly conventional derivation of the Hawking process. This is because any conventional argument must reproduce the propagation of the field assumed by Hawking, and this involves trans-Planckian physics. In other words, in any "conventional" picture, the propagation of the field will be by the same equation as that used by Hawking, and this means that the Hawking quanta would come from field modes propagating arbitrarily close to the event horizon and subject to divergent red-shifts.

There could in principle be, however, an alternative physical argument for

the Hawking process, one which reproduced the trans-Planckian difficulties as an unfortunate by-product but in which one could somehow argue these were not essential. While no argument like this is presently known, the best approach to one goes back to DeWitt (1979) and Unruh (1976), and has ben outlined most explicitly by Jacobson (1996).

According to Unruh, a uniformly accelerated detector in Minkowski space will respond as if it is in a thermal bath. By the principle of equivalence, an observer held fixed in a uniform gravitational field will find physics locally the same as for a uniformly accelerating observer. Thus (one would think) an observer held fixed in a uniform gravitational field will perceive Planckian radiation. This is tantalizingly close to Hawking's prediction. 


\subsection{The Unruh process}

A quick sketch of the Unruh process is in order. While the result is generally accepted, it has not been experimentally verified and has been contested by some workers. (See e.g. Fedotov et al 1999.) I give a treatment in a form which I believe is convincing, but it has been phrased carefully to avoid some of the trickier issues.

Let us assume we have some sort of detector which responds to the quantum field $\phi$. We shall also assume that we can ignore the spatial extent of the detector. ${ }^{12}$ (This is not an entirely trivial assumption, but it is a reasonable first approximation. ${ }^{13}$ ) Then if the detector's world line is $\gamma(s)$ (with $s$ the proper time), it responds to $\phi(\gamma(s))$. In particular, by comparing the two-point function

$$
\left\langle 0_{\mathrm{M}}\left|\phi\left(\gamma\left(s_{1}\right)\right) \phi\left(\gamma\left(s_{2}\right)\right)\right| 0_{\mathrm{M}}\right\rangle
$$

with that of an inertial one

$$
\left\langle 0_{\mathrm{M}}\left|\phi\left(t=s_{1}, x=0, y=0, z=0\right) \phi\left(t=s_{2}, x=0, y=0, z=0\right)\right| 0_{\mathrm{M}}\right\rangle,
$$

we get a measure of whether the state would appear to an observer to be excited beyond the expected vacuum fluctuations.

A world-line with uniform acceleration $a$ in the $x$-direction is

$$
t=a^{-1} \sinh a s, \quad x=a^{-1} \cosh a s, \quad y=0, \quad z=0,
$$

and, on substituting this into the formula (24) for the Minkwoskian two-point function, we find

$$
\begin{aligned}
\left\langle 0_{\mathrm{M}}\right| \phi\left(\gamma\left(s_{1}\right)\right) \phi & \left(\gamma\left(s_{2}\right)\right)\left|0_{\mathrm{M}}\right\rangle \\
& =-\left(4 \pi^{2}\right)^{-1} a^{2}\left(\left(\sinh a s_{1}-\sinh a s_{2}-\mathrm{i} \epsilon\right)^{2}-\left(\cosh a s_{1}-\cosh a s_{2}\right)^{2}\right)^{-1} .
\end{aligned}
$$

This is clearly periodic in imaginary time with period $2 \pi / a$, and one can check that the full KMS condition holds for measurements of the field on the world-line. Thus the state is a thermal one with temperature $T_{\mathrm{U}}=\hbar a /(2 \pi c k)$. The Planckian nature of the spectrum can be established by direct calculation, but it is no surprise because the only dimensionful quantity is the acceleration. Note that the characteristic time to detect a quantum is $\sim c / a$, the acceleration time.

Of course, a world line representing a detector which accelerates uniformly for all time is unrealistic. It would be more natural to consider (say) a detector which was

12 Since particles have finite spatial extent, this means that the detector cannot be in the strict sense a particle detector. It will be a field strength detector.

${ }^{13}$ When the spatial extent of an accelerated detector must be considered, it is necessary to consider how the acceleration varies over the detector. The Unruh analysis (and the Bisognano-Wichmann theorem to be discussed in the next subsection) does not apply to an extended detector all of whose elements suffer the same acceleration, but to those where the acceleration varies in such a way that the spatial separation between adjacent elements is preserved. 
inertial prior to some time, and then was smoothly brought into a state of uniform acceleration, and then eventually smoothly returned to an inertial trajectory. This would not have led to any result differing from Unruh's in the interval of constant acceleration, since what enters is the two-point function (57). Only if the detector were used to measure quanta of such low frequencies that their wave packets extended back to the time prior to the uniform acceleration would discrepancies with Unruh's predictions arise. So for practical purposes, unless very sensitive detections of infrared effects are important, once the uniform acceleration has been sustained over a few acceleration-times' worth of proper time, the spectrum of expected excitations is Planckian.

\subsection{The argument from the equivalence principle}

The argument most nearly connecting the Unruh and Hawking effects goes back to DeWitt (1979) and Unruh (1976) and has more recently been explicitly outlined by Jacobson (1996). Consider an observer hovering outside of a black hole at Schwarzschild coordinate $r$. That observer will experience a local acceleration, which can be computed,

$$
a=f^{-1}(2 M / r)^{2} \kappa,
$$

where $f=\left(1-R_{\mathrm{Sch}} / r\right)^{1 / 2}$ as before. Now on small enough scales near this observer, the two-point function should approach its Minkowskian form, and therefore (applying the principle of equivalence and the Unruh argument) the observer will perceive the vacuum to be at a temperature $T(r)=\hbar a /(2 \pi)$. On account of the red-shift, an observer at infinity would perceive this as $f T(r)$. If we assume that the agreement of the two-point function with its Minkowskian form becomes better as $r \rightarrow R_{\text {Sch }}$, then the temperature of the black hole, as measured at infinity, should be

$$
\lim _{r \downarrow R_{\mathrm{Sch}}} f T(r)=\kappa /(2 \pi)=T_{\mathrm{H}} .
$$

Jacobson outlined this argument with the goal that it would provide an explanation for the Hawking effect without having to invoke physics at the Planck scale (or beyond). The idea was that the trans-Planckian physics would only have to be invoked for observers within the Planck scale of the Schwarzschild radius. However, one would hopefully get a very good approximation to the Hawking picture for observers somewhat farther away. So if the argument held for $r$ separated from $R_{\text {Sch }}$ by a proper distance of $1 \mathrm{~nm}$ (say), one could recover essentially Hawking's prediction while only needing to invoke energies $\sim \hbar c /(1 \mathrm{~nm}) \simeq 200 \mathrm{eV}$. The trans-Planckian regime would still be necessary to describe physics very close to the hole, but hopefully this would simply decouple from the Hawking radiation. Thus one could take the limiting form of the two-point function for $r$ close (but not in Planck terms) to $R_{\text {Sch }}$ as a sort of boundary condition which would give rise to the Hawking effect.

It is clear that this argument turns on the sense in which the two-point function approaches its Minkowskian form. As we shall see quantitatively shortly, the approach 
does not in fact have quite the character hypothesized. First, though, there is larger issue to raise.

The argument is apparently time-symmetric, whereas the Hawking process is not. The only place a time asymmetry might possibly come into the argument would be if the limit which has been written simply as $r \downarrow R_{\text {Sch }}$ must actually be taken in some time-asymmetric way in order to get the correct limiting two-point behavior. If this is not the case, then the argument by appeal to the equivalence principle and Hawking's analysis must speak to different physics. However, even such time-asymmetry in the approach could not be a complete explanation of the discrepancy. If we consider, for example, an object which collapses not quite to a black-hole state but so that its limb is at $r=(1+\epsilon) R_{\text {Sch }}$ and it is stationary, then Hawking's analysis would predict that (after some transients) there would be no production of quanta. However, the argument by appeal to the equivalence principle would predict a Planckian ("Hawking") spectrum.

In order to understand what really is happening, let us consider the situation quantitatively. To make contact with the previous analysis of the Hawking prediction, let us consider a spherically symmetric family of accelerated observers with detectors are arranged to respond only to the $l=0$ modes. Then the Minkowskian null coordinates of the world line of one of these would be $u=-a^{-1} \exp -a s, v=$ $a^{-1} \exp a s$, and the $l=0$ two-point function for the detectors would be (cf. (27))

$$
-\left(16 \pi^{2}\right)^{-1} \log \frac{\left(\mathrm{e}^{a s_{1}}-\mathrm{e}^{a s_{2}}-\mathrm{i} \epsilon\right)\left(-\mathrm{e}^{-a s_{1}}+\mathrm{e}^{-a s_{2}}-\mathrm{i} \epsilon\right)}{\left(\mathrm{e}^{a s_{1}}+\mathrm{e}^{-a s_{2}}-\mathrm{i} \epsilon\right)\left(-\mathrm{e}^{-a s_{1}}-\mathrm{e}^{a s_{2}}-\mathrm{i} \epsilon\right)},
$$

whereas the detectors in the Hawking process would have ${ }^{14}$

$$
-\left(16 \pi^{2}\right)^{-1} \log \frac{\left(v_{1}-v_{2}-\mathrm{i} \epsilon\right)\left(-\mathrm{e}^{-\kappa u_{1}}+\mathrm{e}^{-\kappa u_{2}}-\mathrm{i} \epsilon\right)}{\left(v_{1}+\mathrm{e}^{-\kappa u_{2}}-\mathrm{i} \epsilon\right)\left(-\mathrm{e}^{-\kappa u_{1}}-v_{2}-\mathrm{i} \epsilon\right)} .
$$

In each of these, the important terms are the numerators, since we are interested in what happens when the two events are close and the times are late. If the argument's hypothesis about the two-point asymptotics were correct, we should obtain the second from the first by substituting $\kappa f^{-1}$ for $a$ and $f u$ for $s$. We see that this correctly reproduces the second factor, but not the first. The two forms become asymptotically close (up to constant prefactors) only for $\Delta v \ll a^{-1}$, which is to say $f^{-1} \Delta s \ll a^{-1}$. However, this is just the condition that we are passing the frequencies at which the Unruh quanta occur. This condition is not any more accurately fulfilled as $r \rightarrow R_{\text {Sch }}$. Thus the limiting behavior necessary for the argument, that the two-point function should approach the Minkowskian form sufficiently accurately to locally represent the Unruh process as $r \downarrow R_{\text {Sch }}$, does not hold.

Let us now step back and consider the problem generally. In a curved space-time, in a small enough neighborhood of any event, one expects the two-point function to have the Hadamard form, and this means that in small enough neighborhoods the

${ }^{14}$ Note that we expect the geometric-optics approximation to become exact in this case, as we have $r \downarrow R_{\mathrm{Sch}}$. 
Unruh effect should apply to accelerating observers (those whose acceleration times are small compared to the size of the neighborhood). But what sets the scale at which the Hadamard asymptotics take over? It is the choice of quantum state, for different quantum states have different two-point functions. Thus an argument like DeWitt's really depends on the choice of quantum state (and the state used in the Hawking process does not quite have the required properties).

While the behavior hypothesized in the argument does not hold, the actual behavior is rather close, agreeing in the terms coming from the null coordinate $u$ but disagreeing in those coming from $v$. This is closely related to the possibility of constructing what is called the Hartle-Hawking vacuum.

For us, the lesson to be drawn is that this approach has not succeeded in weakening the dependence of the argument for Hawking radiation on the details of the propagation of the quantum field and the two-point function. We need to know the scale at which the Hadamard asymptotics take over, and this can only be determined by analyzing the propagation of the two-point function.

\subsection{The Bisognano-Wichmann Theorem}

Remarkably, at about the same time Unruh was studying the effects of uniform acceleration on detectors responding to free massless fields, a far more general theorem was being proved in the context of axiomatic field theory. The authors of this theorem, Bisognano and Wichmann (1976), did not mention its interpretation in terms of accelerated observers; they were concerned with it as establishing a duality between certain algebras of observables. The connection with the work of Unruh was pointed out much later, by Sewell (1982).

The Bisognano-Wichmann theorem essentially says that the Unruh effect remains valid for arbitrary, interacting, Poincaré-invariant field theories, in the sense that such a theory with Hamiltonian $H$ will be perceived by a uniformly accelerating observer as if it were in a thermal state with density matrix $\exp -H /(k T)$ where $T=\hbar a /(2 \pi c k)$.

Since the Unruh effect does not explain the Hawking effect, we cannot expect the Bisognano-Wichmann theorem to explain the Hawking effect, either. Nevertheless, the Bisognano-Wichmann theorem is of great interest. It shows that the Unruh effect is profound and not just a peculiarity of an artificially simple field equation; in this way, it also gives hope that if the difficulties with the Hawking effect could be circumvented for linear fields, they might also be circumvented for realistic, interacting, field theories. (See Sewell 1982.)

It is important to point out that there is one, potentially very serious, obstacle to applying arguments like those of Bisognano and Wichmann in curved space-time. This is that the arguments rely essentially on the existence of a self-adjoint semibounded Hamiltonian operator. In a non-stationary space-time, such Hamiltonian operators do not exist (Helfer 1996). Since non-stationarity plays a key role in the Hawking process, such concerns must be kept in mind. 


\section{Lessons from moving-mirror models}

By a moving mirror model, one generally means a linear massless quantum field responding to a perfect reflector in two-dimensional Minkowski space. Such models are closely related to the Hawking process: we noted earlier in the geometric-optics, swave approximation, the evolution of quantum fields in the Hawking model is precisely given by a moving-mirror model (where the mirror's trajectory is given by $v=v(u)$, with $u$ and $v$ interpreted as the Minkowski null coordinates $t-x$ and $t+x)$. Relations

like this one have been used over the years to clarify various aspects of the Hawking process.

While it is sometimes suggested that moving mirror models can explain the Hawking process, this is not really the case. There is a mathematical identity of the scattering of the quantum fields within the two models. The task facing us is to decide in what regimes the models themselves can be justified, and how the physics driving the scattering in one model might be identified with that in another.

What we shall find is that the moving-mirror models suggest that a classical treatment of space-time is inadequate for understanding at least one important aspect of the propagation of quantum fields through gravitationally-collapsing regions, the energy budget of the system. In the moving-mirror models, the energies associated with the quantum fields are typically smaller than the errors made in neglecting the quantum character of the mirror and its driving engine. If similar results hold in the gravitational case, one must question whether the neglect of quantum-gravitational corrections is justified.

\subsection{The trajectories}

Let us begin by seeing what it is which gives rise to the radiation in the movingmirror case, that is, what the trajectory of the mirror is. (It is not one of uniform acceleration.) To understand its structure, let us first work out the proper time along it:

$$
\begin{aligned}
s & =\int \sqrt{\mathrm{d} u \mathrm{~d} v} \\
& =\int \sqrt{v^{\prime}(u)} \mathrm{d} u \\
& \simeq C \exp -\kappa u / 2
\end{aligned}
$$

In what follows, the value of $C$ is irrelevant and we shall take $C=\kappa^{-1}$ to give the simplest form with the correct dimension. Then $s \simeq-\kappa^{-1}\left(v^{\prime}(u)\right)^{1 / 2}$, and $s \uparrow 0$ as $u \rightarrow+\infty$. This means that the mirror moves off to infinity in finite proper time.

The unit timelike tangent to the trajectory is

$$
\begin{aligned}
T^{a} & =\left(v^{\prime}(u)\right)^{-1 / 2}\left(\partial_{u}+v^{\prime}(u) \partial_{v}\right) \\
& \simeq-(s \kappa)^{-1} \partial_{u}-(s \kappa) \partial_{v}
\end{aligned}
$$


The unit normal towards the right is

$$
R^{a} \simeq-(s \kappa)^{-1} \partial_{u}+(s \kappa) \partial_{v}
$$

and the acceleration is

$$
\begin{aligned}
A^{a} & =\frac{\mathrm{d} T^{a}}{d s} \\
& \simeq s^{-2} \kappa^{-1} \partial_{u}-\kappa \partial_{v} \\
& =-s^{-1} R^{a} .
\end{aligned}
$$

Thus as $u \rightarrow+\infty$ and $s \uparrow 0$, the mirror moves to the left, not with constant acceleration, but with acceleration increasing in magnitude unboundedly. In fact, since $T^{a}$ grows unboundedly as $s \uparrow 0$, an infinite amount of energy-momentum must be supplied to the mirror to accelerate it on this trajectory. ${ }^{15}$

At this point, it would not be unreasonable to dismiss the moving-mirror model with this trajectory as hopelessly artificial. Certainly it contrasts grossly with a gravitationally collapsing object, where no external energy at all, certainly not an unbounded one, need be supplied.

There are other concerns about the moving-mirror models as well. Any real mirror is not perfectly reflective, but (roughly speaking) reflects modes only below some plasma frequency $\omega_{\mathrm{p}}$. When the acceleration grows so large that $a \gtrsim c \omega_{\mathrm{p}}$, the velocity is changing appreciably over a time smaller than the response time of the plasmons and so one does not have in any simple sense a mirror. And when $a / c \gtrsim t t_{\mathrm{Pl}}^{-1}$, of course, the neglect of quantum gravity is not credible even in these models.

\subsection{The energy budget}

While we have seen that there are specific concerns about the physical reasonableness of the mirror trajectory used to recover a Hawking-type prediction of Planckian radiation, there is an important lesson to be learned from moving-mirror models as a class, including those with physically reasonable trajectories. This lesson concerns the adequacy of modeling the mirror's trajectory classically for understanding the system's energetics.

If we try to understand the energy budget of a moving-mirror problem, that is, how the mirror can produce quanta by accelerating vacuum fluctuations, we find that it is not adequate to model the mirror classically. The energies of the quanta produced are so low that the uncertainty in the mirror's state due simply to its finite Compton wavelength must be taken into account. In fact, one finds that when the mirror accelerates, its state (and that of the engine driving it) become entangled in a significant way with the vacuum fluctuations in question, and so the mirror (and its driving engine) are in a superposition of energy states whose width is of the order

15 While $T^{a}$ remains a unit vector, on account of the Lorentzian signature it can still increase unboundedly, moving out to infinity along the unit hyperbola. 
of the energy of the quanta created from the vacuum fluctuations (Parentani 1996, Helfer 2001a).

The main idea of the argument can be given as follows. Consider a (for simplicity, non-relativistic) mirror of mass $m$, moving in a potential $V(x)$. Then the Hamiltonian for the system (of mirror and field) has the form

$$
H_{\text {mirror }}+H_{\text {field, left }}+H_{\text {field, right }} \text {. }
$$

Here

$$
H_{\text {mirror }}=\frac{p^{2}}{2 m}+V(x) \text {, }
$$

and $H_{\text {field, left }}, H_{\text {field, right }}$ are the Hamiltonians of the field on either side of the mirror. Each of the field Hamiltonians has a zero-point term of magnitude

$$
(12 \pi)^{-1}(\hbar / m c) V^{\prime}(x)
$$

and represents the excitation of the field by the mirror even for a vacuum in-state (the case of interest here).

Note that the zero-point term could be interpreted as arising from a displacement of the coordinate in the potential term $V(x)$ by an amount $\Delta x=(12 \pi)^{-1}(\hbar / m c)$. Now $\hbar / m c$ is the mirror's Compton wavelength, that is, the scale at which the mirror cannot be considered as (even) a (quantum) point particle, but must be given a relativistic quantum field-theoretic treatment. (This smearing-out of the point on which a potential acts is a general feature of relativistic quantum field theories, usually discussed under the heading "Zitterbewegung.")

A few comments are in order. First, while the argument has been phrased simply in terms of a "mirror," it really refers to the reflective agents, presumably plasmons

in a more realistic treatment. Second, it is rather a surprise that one needs to pass to a relativistic quantum treatment of the mirror. That one must do so is a measure of the delicacy of the energetics of the system.

In the parallel with the Hawking process, the mirror corresponds to the gravitationally collapsing space-time. Thus if the analogy between the two systems holds, in order to have a treatment of the Hawking system adequate for the accounting of energy transfers between the collapsing system and the quantum field, we will need a relativistic quantum treatment of the collapsing geometry: a quantum-gravitational treatment.

\section{Connections with thermodynamics}

Before Hawking's prediction of black-hole evaporation, a close formal similarity between the classical general-relativistic theory of black holes and thermodynamics had been noted. It was regarded by most workers, though, as only formal (see however Bekenstein 1973). With Hawking's prediction that black holes were indeed 
thermodynamic objects, there seemed to be good reason to suppose that the connection was very deep indeed. Subsequent work has focused on elaborating this connection. The theory developed is sometimes cited as the strongest evidence, albeit circumstantial, that the Hawking effect is real. The sentiment is that the overall picture is so attractive, the details will somehow sort themselves out to produce the evaporation predicted by Hawking.

This sentiment, while perfectly legitimate, is not really of itself an argument that the Hawking effect is real, but rather an argument for which way one should guess is it real or not? Such guesses are a reasonable and often necessary part of theoretical physics, but they are not substitutes for trying to sort out what really happens.

All of the work to be described in this section will be of this circumstantial character, and thus will not directly address the trans-Planckian problem or the question of quantum-gravitational corrections. I shall only raise these issues explicitly, though, when they arise in senses not yet encountered.

How good is this overall picture of the Hawking effect together with thermodynamics? The real answer is that the picture must still be considered to be in a stage of development. There are good arguments that the Hawking effect should respect the "generalized second law." On the other hand, we shall see that there are difficulties in making precise exactly what this generalized second law says, and some question about the arguments that there is a need to generalize the second law. We shall also see that there are some important open issues in the analyses of some of the thought-experiments proposed to test the generalized second law. Thus, while there is indeed a general overall related set of analyses involving the Hawking effect and thermodynamics integrally, there are significant unresolved issues, in establishing the results and even in formulating them.

While the work in this area is not at present definitive, it is some of the most important in the field. It attempts to build detailed physical pictures of what happens, and it is from attempts to model real situations that we can hope to learn the most.

I will close this introductory section with an only half-way tongue-in-cheek comment of Eddington's, which brings out both the physicist's conviction of the primacy of the second law ${ }^{16}$ and the distinction between whether one has actually analyzed a conflict in physical theories or simply has a strong belief in which way it will be resolved.

If someone points out to you that your pet theory of the universe is in disagreement with Maxwell's equations - then so much the worse for Maxwell's equations. If it is found to be contradicted by observation - well, these experimentalists do bungle things sometimes. But if your theory is found to be against the second law of thermodynamics I can give you no hope; there is nothing for it but to collapse in deepest humiliation. (Eddington 1929)

${ }^{16}$ A notion which should be given some thought. 


\subsection{Classical black-hole thermodynamics}

We begin by summarizing what is called classical black-hole thermodynamics. Whether it really is thermodynamics or not is not wholly clear, and indeed prior to Hawking's prediction of black-hole radiation it was viewed by most workers as simply an analogy. However, the formal similarities are so striking that it is reasonable to conjecture that there is a deep connection, remaining to be understood.

The zeroth law is that an isolated, stationary black hole has a well-defined "temperature," its surface gravity $\kappa$. That is, the surface gravity of an isolated, stationary black hole is constant over the event horizon. This is not at all a trivial result for non-spherically symmetric holes. Note that, without bringing in Planck's constant, it is not possible to convert $\kappa$ to a quantity with units temperature (or energy); dimensionally a temperature would be $\hbar \kappa /(c k)$.

The first law is conservation of energy, which can be expressed in differential form as $8 \pi\left(c^{2} / G\right) \kappa \mathrm{d} A=\mathrm{d} M c^{2}-\Omega \mathrm{d} J-\Phi \mathrm{d} Q$ (with $\Omega$ and $\Phi$ the angular velocity and electromagnetic potential at the horizon), giving the response of the hole to changes in charge, mass and angular momentum; it parallels a thermodynamic $k T \mathrm{~d} S=\mathrm{d} U-\Omega \mathrm{d} J-\Phi \mathrm{d} Q$.

The second law is the statement that, if the energy density of matter crossing the horizon is positive, then the area of the black hole cannot decrease. (This is Hawking's celebrated Area Theorem.) Thus one identifies the area with entropy. Again, classically these have different units, and to convert area into an entropy one must form $A k c /(\hbar G)=k A / l_{\mathrm{Pl}}^{2}$. Note that the factor $l_{\mathrm{Pl}}^{-2}$ will give even black holes of modest size colossal entropies.

While these results are very beautiful, they have no obvious connection with ordinary thermodynamics. In particular, neither the "temperature" $\kappa$ nor the "entropy" $A$ has any clear thermodynamic interpretation or significance.

Hawking's prediction gives an obvious thermodynamic interpretation to $\kappa$ (or $\kappa / 2 \pi)$ as the temperature. It does not give quite so obvious a thermodynamic interpretation to the area as the entropy, although such an interpretation can be inferred from the classical first law. We shall see later in this section that there is a certain amount of evidence that this interpretation is consistent with other thermodynamic principles. On the other hand, the problem of finding a convincing explanation of the identification of area with entropy (for example, by identifying the black hole's area as a measure of the logarithm of some volume of phase space) is not addressed by most of these considerations. It may be addressed by quantumgravitational computations, however, as will be discussed in section 10 .

\subsection{General relativity and the second law}

A main motivation for the generalized second law was a concern that the ordinary second law of thermodynamics might not hold in general relativity. The argument runs as follows. 
Imagine adiabatically lowering a box of gas (or thermal radiation) towards a Schwarzschild black hole. As it is lowered, potential energy is recovered, this energy corresponding to the red-shift of the box's energy relative to infinity. The entire energy of the box is red-shifted, including its heat content. Thus as one lowers the box towards the black-hole horizon, one recovers a fraction arbitrarily close to unity of the energy in the box as useful energy at infinity. This process seems to contradict the second law, at least in its ordinary sense, for it allows one to convert thermal energy to work adiabatically. This argument is often cited as a demonstration that classical general relativity is not compatible with the second law. It is therefore taken as a sign that the second law will have to be modified if it is to hold when black holes are present. ${ }^{17}$

However, on more careful consideration, it is not clear that the second law really has been violated. Even if one lowers the box to within a proper distance $\epsilon R_{\text {Sch }}$ of the hole and then releases it, external observers will never see the box, or the entropy it contains, enter the hole. They see the box, very red-shifted, moving towards the Schwarzschild radius exponentially slowly. ${ }^{18}$ No entropy has been lost, even as far as simply the region outside of the hole is concerned. (On the other hand, one should note that, given that the box will fall into the hole, it is beyond the influence of distant observers.)

There is another issue. This is that one would not expect the local temperature, and hence the local energy, of the box to remain exactly constant as the box is lowered adiabatically. This is because the gravitational field will tend to pull matter and radiation within the box towards its bottom. This inhomogeneity would decrease the entropy, and would have to be offset in an adiabatic process by an increase in temperature (assuming positive specific heat) and internal energy. This means one cannot extract quite as much energy, for an increment of motion of the box, as thought naively. There might even be a point, somewhat before the Schwarzschild radius is reached, at which the maximum energy extraction for an adiabatic process is reached. Lowering the box further would require either abandoning the adiabatic condition, or actually putting energy into the box. In other words, the requirement of adiabaticity might lead to a sort of flotation point. A similar but not identical behavior, in the quantum analysis, was noted by Unruh and Wald (1982) (see also Radzikowki and Unruh 1988, which contains a correction); see section 7.5.

It should also be pointed out that in these models the black hole has been treated as being at zero temperature. The validity of this needs to be justified, as examples like Feynman's ratchet and pawl (or Maxwell's demon) show.

17 The process described here, of lowering a thermodynamic system towards a black hole, has come to be called by some the Geroch process, and by others the Wheeler process. It was apparently described by both men, independently, at Princeton around 1971 (Bekenstein 1973). However, no published work by Wheeler or Geroch on this seems to survive.

18 This analysis assumes the cosmic censorship conjecture. Were the conjecture to fail, distant observers might see the box disappear as it went behind a naked singularity to cross the horizon. 
In the literature, there is much discussion of "dropping things (or entropy) into black holes." The assertion is made that in this process entropy is hidden from the outside world, and that for this reason the second law appears to be violated. However, this assertion really comes from combining selectively chosen elements of the physics as perceived by different observers in a way which cannot be expected to provide an accurate accounting for the entropy of the system. On the one had, the authors want to consider a family of observers, some of whom fall into the hole, in order to be able to assert that the box has fallen in; on the other, they want to only consider the entropy measured by those observers in their family who stay outside the hole. ${ }^{19}$

At a deeper level, it is not wholly clear how one should formulate the second law in even the simplest black-hole space-times: whether it should refer only to region external to the hole, or take into account the hole as well. One must also bear in mind that inside the hole (and, for rotating black holes, inside the "ergosphere"), no observer sees space-time to be stationary. This means that what is usually taken as a basic underpinning of thermodynamics is absent.

In sum, the situation is that there is no clear violation of the second law, but also some concern about what the precise formulation of the second law should be.

\subsection{The generalized second law}

As just pointed out, the argument that "dropping thermal systems into black holes leads to violations of the second law" is probably not really correct. However, this does not seem to go to the root of the concern about entropy and event horizons.

The root issue is that (minus) the entropy represents a measure of information, and information is in some sense lost to the external world when it crosses an event horizon. Thus there does seem to be an important and perhaps profound physical problem: to find an extension of the second law which provides an accounting of entropy for families of observers up to and including those on an event horizon. The generalized second law is a hypothesized extension of this sort, first proposed by Bekenstein (1973):

The common entropy in the black-hole exterior plus the black-hole entropy never decreases.

(With the understanding that the black-hole entropy is a constant multiple of the area. $)^{20}$

19 The question of whether a box can be considered "for all practical purposes" to be in the black hole is bound up with the choice of coarse-graining scale. A choice of coarse-graining scale presumably defines a thickened "effective" horizon such that any object which comes within this thickened region is considered for practical purposes to be part of the horizon. However, this scale must also be finer than the scale on which one wants to consider the degrees of freedom of the thermal system in the box. Thus one cannot bring the entire box, or even a significant fraction of it, within the thickened effective horizon.

${ }^{20}$ If one wants to retain the original motivation for the generalized second law (loss of entropy from the external world, etc.), and accepts the comments I have made earlier to the effect that violations of the ordinary second law are only possible when naked singularities appear, then one would presumably conjecture that the common entropy in the exterior of a singularity, plus the area of the singularity (however that might be defined) never decreases. 
It is worthwhile discussing what the precise mathematical and physical formulation of this ought to be. It seems reasonable that we should measure the entropy on some "surface of constant time," a Cauchy surface from which the interiors of any black holes have been excised. "Never decreasing" has a clear meaning, then, for there is a clear sense in which one such surface is to the future of another.

It is less clear what "common entropy" should be, because of the difficulty in defining entropy for very general systems. It is also not wholly clear, in the most general circumstances, what it means to simply consider the entropy "in the exterior," for there may be correlations which relate interior and exterior modes.

Would the Geroch-Wheeler process obey the generalized second law? At first sight, it is not at all clear that it could. If we ignore the buoyancy effect I described earlier, then one could lower a box of entropy arbitrarily close to the Schwarzschild radius of a hole. This would render the box's contribution to the hole's mass (when the box is finally dropped in) arbitrarily small, so it would not increase the hole's entropy. Yet the entropy of the box would cross the horizon, violating the generalized second law.

This issue was recognized by Bekenstein (1973) from the first, and he proposed that the generalized second law would be enforced by previously unsuspected entropy bounds, of the form

$$
S \leq 2 \pi R E /(\hbar c),
$$

where $E$ and $R$ are the energy and characteristic dimension of the box, and $S$ is the entropy it contains. Since then, there has been much thought given to the problem of establishing entropy bounds of various sorts. (See Bousso 2002 for a review.) At present there is not agreement, however, that strong enough bounds have been proved to enforce the generalized second law. However, the buoyancy concern that I raised above, and one raised earlier by Unruh and Wald (1983), also raise the possibility that the generalized second law could be enforced by physics other than entropy bounds. (See Bekenstein 1982, 1994b, 2002, and Wald 2001 for discussion of the debate over entropy bounds.)

In any event, it should be noted that when ordinary macroscopic objects cross the event horizon, the gain in black-hole entropy is typically colossal compared to any common entropy involved. For example, a $1 \mathrm{~kg}$ mass falling into a solar-mass black hole would increase the black-hole entropy by $\sim 10^{47}$, whereas the common entropy of the mass would be (very roughly) the number of baryons, $\sim 10^{27}$, many orders of magnitude smaller. It is hard to think of ways of violating the generalized second law with ordinary sorts of matter.

A related point is that if indeed it were possible to violate the ordinary second law (whatever that means in a general-relativistic context) up to the limits imposed by the generalized second law, those violations would be enormous. For the loss in ordinary entropy would be the gain in black-hole entropy.

Note however that if the area of the black hole could decrease (this would correspond to the black hole gaining negative energy), the requirement that the 
generalized second law hold might be much more difficult to meet. If somehow a $-1 \mathrm{~kg}$ mass could be dropped into a solar-mass black hole, the generalized second law would require this to be accompanied by a huge $\left(\sim 10^{47}\right)$ increase in the entropy of the exterior. Of course, we do not have any $-1 \mathrm{~kg}$ masses. But even with the tiny negative energies supposed to be absorbed by the black hole in the Hawking process, we have a non-trivial test of the generalized second law, as we now discuss.

\subsection{The generalized second law and the Hawking process}

Does the Hawking process respect the generalized second law? There is good (although not quite conclusive) evidence that it does. And the picture that emerges is in some respects quite appealing, and is therefore taken to be circumstantial evidence for the Hawking process.

There are two competing effects which need to be considered in order to check whether the Hawking process respects the generalized second law. On the one hand, the state of the quantum field changes, and in particular thermal radiation is emitted

to infinity. While precisely how the field's state, and entropy, change need to be thought about carefully, one would expect an overall increase in "common" entropy from this. On the other hand, since (presumably) energy is conserved, the black hole itself must be losing mass in the Hawking process. This means its area, and hence the black-hole entropy, should be decreasing.

\subsubsection{Quantum-gravitational issues.}

It should be noted that any assumption about how the black hole loses mass implicitly involves quantum-gravitational assumptions, if only in a negative way. (By negative quantum-gravitational assumptions, I mean that one assumes that classical general relativity is adequate when dimensional or other arguments show that there are some potentially quantum-gravitational effects.) Since the emission of Hawking radiation is a highly non-classical process, the question of how the quanta carry away energy is potentially one, too. However, if Hawking radiation does exist, it is probably legitimate to approximate the time-averaged energy loss by a semiclassical source term $\left\langle T_{a b}\right\rangle$. (Here time-averaged means over scales $\gtrsim G M / c^{3}$, the characteristic time to emit a Hawking quantum.) This would lead to a mass loss of the hole due precisely to the expected Hawking flux. This is the assumption generally made.

It turns out that one must consider still longer averaging times, though, to avoid potential quantum-gravitational effects. Neglecting numerical factors, the mass loss of the hole in a time $\sim G M / c^{3}$ (the characteristic time to emit a Hawking quantum) will be $\sim k T_{\mathrm{H}} / c^{2} \sim m_{\mathrm{Pl}}^{2} / M$. This means that the change in Schwarzschild radius should be $\sim l_{\mathrm{Pl}} m_{\mathrm{Pl}} / M$, a tiny fraction of the Planck length. It is unrealistic to assume that changes like this have any meaning - it is probably unrealistic to believe they have any meaning. A classical model of space-time is thus not clearly credible for the analysis of such processes. Only if we average over times $\gtrsim\left(M / m_{\mathrm{Pl}}\right)\left(G M / c^{3}\right)$ does 
the change in Schwarzschild radius approach the Planck length and a classical general-relativistic picture of the change in the horizon become credible. ${ }^{21}$ For a solar-mass black hole, this longer averaging time would be $\sim 10^{25} \mathrm{y}$, but for a $10^{15} \mathrm{~g}$ "mini" black hole, it would be $\sim 10^{-4} \mathrm{~s}$.

\subsubsection{Definition of entropy.}

In principle, one would like to compute the field entropy on a partial Cauchy surface in the exterior of the hole, and see how this changes as the surface moves forwards in time. Some progress has been made towards this goal, by taking the field entropy to be the entanglement entropy of the modes outside the black hole relative to those inside (Bombelli et al. 1986, Bekenstein 1994a). However, the definition of this involves some sort of quantum-field-theoretic regularization, and a full understanding of this is yet to come. In particular, it is not clear if one will be able to avoid the use of trans-Planckian cutoffs.

\subsubsection{Rate of entropy production.}

Another line of approach is to compute the average rate of entropy production in the radiation. This would be simply be given by the entropy for blackbody radiation, were the spectrum exactly thermal. On account of deviations from the Planckian spectrum, however, there are modifications to the blackbody formula. Remarkably, however, there is a general argument that the rate of entropy production on account of radiation exceeds the rate of area loss (Bekenstein 1975, Panangaden and Wald 1977). Page (1976) has found numerically that (depending on the species) the excess is typically by a factor $\sim 1.5$. This line of argument can be extended to take into account non-vacuum in-states; see Frolov and Page (1993) (as well as the related paper of Hawking 1976).

In the form just given, the radiative-entropy calculations are a little removed from verifying the generalized second law in the form I have given it. This is because the entropy of the radiation is not the entire entropy (on any partial Cauchy surface) of the region exterior to the black hole. However, it is possible to use these calculations to get a result which is close to verifying the second law in this strong sense. This is not quite trivial, because one has to deal with the problem of defining entropy on the partial Cauchy surface, as well as the fact that correlations between interior modes and radiative modes potentially render entropy not an extensive quantity.

However, it is possible to phrase things so as to largely, although not completely, dodge these difficulties. The idea is this. Consider a partial Cauchy surface $\Sigma$ consisting of two parts, one a spacelike surface $\Gamma$ extending from the event horizon to $\mathcal{I}^{+}$, and the second the portion $\mathcal{N}$ of $\mathcal{I}^{+}$to the past of $\Gamma$. We assume that $\Sigma=\Gamma \cup \mathcal{N}$ lies to the future of the collapsing matter, so that it is in the stationary portion of the

21 Over a time $G M / c^{3}$, the area changes by $\sim l_{\mathrm{Pl}}^{2}$, and so one could argue that the change in area has classical meaning over such times. However, the point made here is that one does not have a full classical general-relativistic picture over such time scales. Thus we do not really know that we are justified in modeling space-time classically for these purposes. 
Schwarzschild solution, and we let $\Sigma_{t}=\Gamma_{t} \cup \mathcal{N}_{t}$ be the surface got by flowing forward by an amount $t>0$ along the vector field $\partial / \partial t$. (See figure 3.)

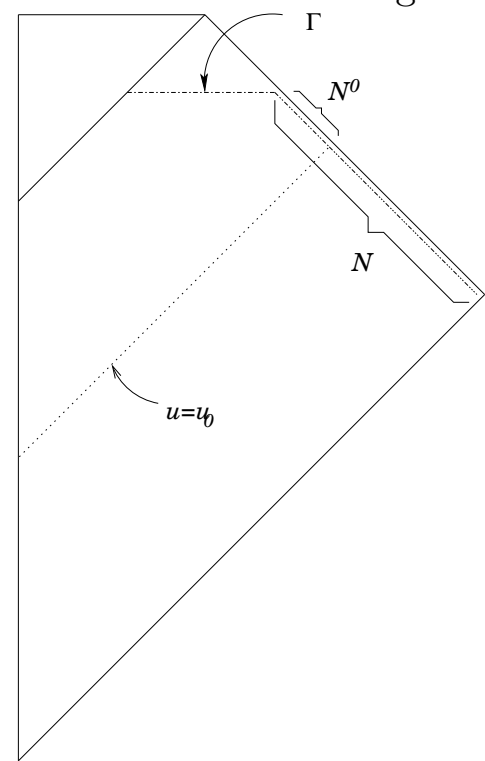

Figure 3. The geometry of the partial Cauchy surfaces used in the argument that the rate-of-entropy-production calculations support the strong form of the generalized second law. The partial Cauchy surface $\Sigma$ consists of two parts, an "internal" $\Gamma$, and a portion $\mathcal{N}$ sensitive to the radiative data. Then $\mathcal{N}_{0}$ is the portion of $\mathcal{N}$ to the future of the retarded time $u=u_{0}$ at which the Hawking radiation nominally starts. The definitions for $\Sigma_{t}$ are got by flowing all quantities except $u=u_{0}$ forward along the Killing field.

There will be some nominal retarded time $u_{0}$ at which the Hawking process may be said to have begun (and transient behavior passed). We shall assume that we can neglect correlations between the field for the portions of $\Sigma\left(\right.$ or $\left.\Sigma_{t}\right)$ before and after $u=u_{0}$ in calculating the entropy. Then to check the generalized second law, we must compute the difference in field entropies

$$
S\left(\Gamma_{t} \cup \mathcal{N}_{t}^{0}\right)-S\left(\Gamma \cup \mathcal{N}^{0}\right),
$$

where $\mathcal{N}_{t}^{0}=\left\{(u, \theta, \varphi) \in \mathcal{N}_{t} \mid u \geq u_{0}\right\}$, and similarly for $\mathcal{N}^{0}$. On the other hand, since the Hawking process is stationary, the entropies will be the same for that portion of $\Gamma_{t} \cup \mathcal{N}_{t}^{0}$ which is the image of $\Gamma_{t} \cup \mathcal{N}^{0}$. Now since the radiation is thermal, it is a good approximation to treat the entropy within the portion of $\mathcal{I}^{+}$receiving the radiation as an extensive quantity. It is also, we assume, a good approximation to treat the field on

$$
A_{t}=\left\{(u, \theta, \varphi) \mid u_{0} \leq u \leq u_{0}+t\right\}
$$

as uncorrelated with that on $\Gamma_{t}$ - indeed, it seems likely that this assumption becomes better for large $t$, since $A_{t}$ becomes larger but $\Gamma_{t}$ stays the same size. Under this assumption, we have

$$
S\left(\Gamma_{t} \cup \mathcal{N}_{t}^{0}\right)-S\left(\Gamma \cup \mathcal{N}^{0}\right)=S\left(A_{t}\right) .
$$

This is exactly the radiative entropy. 
A number of comments on this argument: (a) The assumptions made about correlations seem plausible physically. (b) It dodges the technically difficult issue of defining entropy over $\Sigma$ and relies on the stationarity of the space-time to cancel out "internal" (on $\Gamma$ and $\Gamma_{t}$ ) portions of the entropy. (c) It is not clear that the space-time really is stationary to the accuracy required.

\subsection{The Geroch-Wheeler process revisited}

The Geroch-Wheeler process was reconsidered by Unruh and Wald (1982) (see also the correction, Radzikowski and Unruh 1988) in the light of quantum theory. They discovered a number of very interesting phenomena which suggested a coherent picture of the generalized second law, the Hawking process, and Unruh radiation. Their aim of developing an explicit physical picture serves as one of the most important models in the area.

What Unruh and Wald found was that it was necessary to take into account a variety of factors involving zero-point fluctuations. It was necessary to consider not just the Hawking radiation, but also moving-mirror effects induced by the walls of the box and (at least for observers comoving with the box) Unruh radiation. While there are some questions concerning the hypotheses of the analysis (and hence its conclusions), it is clear that any future work will have to face up to the issues these authors uncovered. If anything, the problem may be still more delicate.

Unruh and Wald actually considered, not quite the Hawking process, but a Hawking-radiating black hole in equilibrium with thermal radiation at temperature $T_{\mathrm{H}}$; this is known as the Hartle-Hawking state. And they actually analyzed the problem from two different points of view: that of an observer comoving with the box (hence accelerating); and that of an inertial observer.

From the point of view of the comoving observer, they found that the Unruh radiation impinging on the box exerted a buoyant force, which became so strong as to cause the box to float at a certain point. Thus there was never any question of dropping the box into the black hole, and in considering various possibilities for opening and shutting and raising and lowering the box Unruh and Wald found no violation of the generalized second law. For inertial observers, the analysis is more difficult, and Unruh and Wald relied on two-dimensional model calculations. These suggested that the buoyant force could be thought of as arising from radiation induced by the motion of the reflecting walls of the box.

There are two, related, concerns about the Unruh-Wald analysis. The first is that it is semiclassical. For example, the radiation pressure on a wall of the box is taken to be a c-number, whereas actually it is a distribution-valued operator sensitive to the scale on which it is measured. This simply means that a small box cannot detect the contributions of the long-wavelength field modes to the pressure. As Bekenstein (1999) has pointed out, the scales involved are such that this may be a serious concern. (See also Bekenstein 2002.) 
It is also a bit dubious to give a semiclassical analysis which involves fictional Unruh observers (that is, accelerated observers who do not actually make measurements of the quantum field but merely aim to describe it mathematically). This is because the energy for the Unruh process, when it is a real process (that is, quanta are really detected), must presumably be supplied by the engine which accelerates the observers. The process inevitably entangles the field's state with that of the motive engine, and so it is not clear whether a semiclassical analysis is adequate.

The second concern is best introduced by pointing out that Unruh and Wald themselves deduced from their analysis that an empty box, once accelerated to a certain point, would maintain its acceleration, by mining energy from the quantum vacuum. Clearly, this is a provocative result and one which needs to be investigated carefully. Recently, Marolf and Sorkin (2002) have argued that the result is unrealistic, and that a realistic treatment would require a quantization of the internal mirror modes (and does not seem to lead to self-acceleration).

The Marolf-Sorkin analysis is consonant with findings in other moving-mirror models (Parentani 1996, Helfer 2001a) (cf. section 6.2). It seems that in order to have an accurate enough treatment of these to analyze second-law issues arising from vacuum fluctuations, one needs to treat the mirrors as quantum objects, and indeed to second-quantize the reflective mirror modes (the plasmons). In Helfer (2001a), it was found that these limitations invalidated some attempts to defeat the (ordinary) second law.

At present, a convincing treatment of the Geroch-Wheeler process at this level has not been achieved. As Marolf and Sorkin charmingly put it, when they analyzed the process in light of their conclusions,

$[\mathrm{O}]$ ne is forced to consider temperatures at which the "thermal radiation" is dominated by box-antibox pairs. This clouds the picture somewhat.

\section{Nonstandard propagation}

Describing the appearance of trans-Planckian modes in the usual Hawking calculation as "certainly wrong," Unruh (1995) considered what might be a substitute and discovered one of the most curious results in the area. Motivated by the physics of sound waves at very short wavelengths, he investigated a model with where there was a limiting ultraviolet frequency $\omega_{0}$, and dispersive propagation at very high wave numbers. He found significant evidence for thermal creation of particles in this model, at the model's analog of the Hawking temperature. Subsequent investigations of this and related models have found similar results.

This area is very much a matter for investigation. Some aspects of the physics are now well-understood, but other essential points are not yet clear. Also various models with different features have been considered, and there is at present no consensus about which (if any) is preferred. Finally, the successes of these models in dealing with the trans-Planckian problem are unclear. Most require ultrahigh wave-numbers 
although they have bounded frequencies. Some of the models of Corley and Jacobson (1996) avoid both ultrahigh wave-numbers and ultrahigh frequencies, but these have apparently unphysical features. There also exist models where the field is confined to a lattice in a black-hole space-time (Corley and Jacobson 1998, Jacobson and Mattingly 2000), but so far these seem to involve either trans-Planckian modes or physically unacceptable distortions of the lattice.

Before discussing the Unruh-type models, I shall review a related proposal of Jacobson (1993). This was an attempt to modify the propagation by introducing a boundary condition which would do away with trans-Planckian problems. Then I shall briefly discuss two of the Unruh-type models, one due to Corley and Jacobson (1996) and the other to Brout, Massar, Parentani and Spindel (1995b) (BMPS). There is a large overlap between these two, and between them and Unruh's original work, so substantially the same comments apply to all. ${ }^{22}$

All models considered so far are in two space-time dimensions, and presumably connect with the Hawking process by describing the s-wave sector.

\subsection{Jacobson's cut-off model}

Jacobson (1993) introduced a model which can be viewed in several different ways: it gives an illustration of the localization of the trans-Planckian problem discussed in section 4.1; it can be thought of as an alternative hypothesis for reproducing Hawking's predictions; it overlaps to a degree with Unruh's ideas. It is based on the introduction of a non-standard boundary condition, and so - propagation being determined by a field equation and boundary conditions - is also really an example of non-standard propagation.

For our purposes, it is best to present this model from a perspective a little different from the original one; comments on the original will be given in the course of the discussion.

Let us fix a radius $r_{\mathrm{bc}}=(1+\epsilon) R_{\mathrm{Sch}}$, with $\epsilon$ small but not so small that observers freely falling across the horizon (from zero velocity at infinity) would perceive trans-

Planckian frequencies in the propagation of Hawking quanta for $r \geq r_{\mathrm{bc}}$. If one then simply takes as data at this boundary the Hawking state (properly, its $n$-point functions and field operators), one can regard the region $r \geq r_{\mathrm{bc}}$, with this boundary condition, as a Hawking-radiating space-time for which no trans-Planckian problem arises. (One can therefore introduce a cis-Planckian cut-off without affecting the predictions, so this is sometimes called a cut-off model.)

So far, we have simply cut out from space-time the trans-Planckian regime near the event horizon; one can think of this as just an illustration of the localization of the

${ }^{22}$ Unruh's model is not discussed directly here because it involves a spatial periodicity. While this periodicity is not really essential to the issues dealt with here, explaining just why this is, when the asymptotic structures of black-hole space-times are so much in the foreground, would require a lengthy and perhaps distracting treatment. 
trans-Planckian problem there. However, Jacobson suggests we take the boundary condition at $r=r_{\mathrm{bc}}$ as a new law of physics.

This is reasonable, especially if one believes that Hawking radiation really does occur but that there are problems with the trans-Planckian physics in its derivation. It cannot be counted as a final theory, however, for it does not really address what the physics is for $r \leq r_{\mathrm{bc}}$, and also one would really like some sort of an explanation of what deeper physics might give rise to this boundary condition. While these concerns were certainly known to Jacobson, it is appropriate to spell them out, and raise some related points.

Since the horizon is determined by global data, one would like to know how the space-time "knows" to implement the boundary condition. Does the boundary condition really only apply when an actual black hole is to form, or would it also apply for "almost-black holes," that is, for objects collapsing to $r=\left(1+\epsilon^{\prime}\right) R_{\text {Sch }}$ ?

The boundary condition is imposed on a timelike surface. In general, such boundary conditions lead to sensitive dependence on initial data. (A simple example of this in Minkowski space, for the massless field and the surface $z=0$, is

$$
\phi=a \mathrm{e}^{\mathrm{i} \omega t-\mathrm{i} k x} \cosh (\kappa z)
$$

with

$$
\omega^{2}-k^{2}+\kappa^{2}=0
$$

By choosing $|a|$ small, the data $\left.\left(\phi, \partial_{z} \phi\right)\right|_{z=0}$ can be chosen uniformly small. However, one can arrange for $\kappa$ to be arbitrarily large, which makes $\phi$ arbitrarily large in any given neighborhood of $z=0$.) This issue has not yet been investigated. On the other hand, much of Jacobson's paper is concerned with a careful argument to the effect that his boundary condition has an internal self-consistency: it can perpetuate itself without any reference to ultra-high frequency physics. Perhaps developments of these ideas could address the sensitivity issue.

There is also the question of the trans-Planckian frequencies associated with the advanced time of formation $v=v_{0}$ of the hole (that is, with the extension of the generators of the event horizon backwards through space-time). In other words, the model does not yet seem to address the fact that the Hawking quanta have their origins in trans-Planckian vacuum fluctuations near $v=v_{0}$.

Finally, it should be mentioned that Jacobson considered it quite possible that his boundary condition would not be fulfilled, and that there might therefore be modifications to the Hawking flux.

\subsection{The model of Corley and Jacobson}

The main features of the two models to be discussed (that of Corley and Jacobson, and that of BMPS.) are similar. I shall give a sketch of the Corley-Jacobson model here, and in the next subsection only describe the essential differences of the BMPS model. 


\subsubsection{The space-times.}

The models considered are space-times with metrics

$$
\mathrm{d} s^{2}=\mathrm{d} t^{2}-(\mathrm{d} x-v(x) \mathrm{d} t)^{2} .
$$

(The reader is cautioned that the symbols used for the coordinates by different authors are not compatible.) The Schwarzschild metric (modulo its angular parts) is included in these, the case $v(x)=-\sqrt{R_{\mathrm{Sch}} / x}$. We shall assume $v(x)$ is an increasing, continuously differentiable, function of $x$, which tends to a constant value (less than unity) as $x \rightarrow+\infty$. Then the metric is regular everywhere throughout the $(t, x)$ coordinate system. The vector field $\partial_{x}$ is everywhere space-like, but $\partial_{t}$ is timelike iff $|v|<1$. The temporal orientation is taken by defining $\partial_{t}$ to be future-pointing in the region $|v|<1$; elsewhere, it is determined by continuity. We shall mostly be interested in the region $v \geq-1$.

The curve $v=-1$ is null and is a Killing horizon. (That is, the Killing vector $\partial_{t}$ is tangent to it.) It is also, in a suitable sense, an event horizon. Precisely, we define a null curve to escape to future right infinity $\mathcal{I}_{\mathrm{r}}^{+}$if $x$ increases without bound towards the future. (Because of the assumptions made on $v(x)$, this will agree with any other standard definition of the "right half" of $\mathcal{I}^{+}$in this two-dimensional space-time.) Then $v=-1$ is the boundary of the set of events from which there are causal curves to $\mathcal{I}_{\mathrm{r}}^{+}$, as is easily checked from the differential equation for the null curves. We may thus call it the future right event horizon. It will be the analog, in these models, of the event horizon of the black hole.

The curves $\mathrm{d} x=v \mathrm{~d} t$ are the trajectories of freely-falling observers, everywhere orthogonal to the surfaces $t=$ const, and crossing the horizon transversely. They play an important role in the models, as the sense of what the "in-vacuum" is taken to be will be defined in terms of these. Note that this is (at least on its face) a highly coordinate-dependent concept. And even if $v$ tends to zero asymptotically, the $(t, x)$ coordinates need not be inertial. For example, in the Schwarzschild case, we have $v(x)=-\sqrt{R_{\mathrm{Sch}} / x}$, and so the trajectories are $-(2 / 3) \sqrt{x^{3} / R_{\mathrm{Sch}}}=c t+$ const.

It should be emphasized that in the Corley-Jacobson (and in the Unruh) models, the space-time region that is examined is exactly stationary. It is supposed to model space-time after gravitational collapse has occurred, and no input about the collapse, or the propagation of the quantum fields through the collapsing region, is used. In this way it differs essentially from the Hawking model.

\subsubsection{The dispersive propagation.}

Even in Minkowski space, dispersive propagation is not Lorentz-invariant. Physically, this is due to the fact that the dispersive medium defines a preferred frame. In order to define the propagation in the Unruh-type models, one must break local Lorentz invariance. The authors do this by writing their propagation equation as

$$
\left(\partial_{t}+\partial_{x} v\right)\left(\partial_{t}+v \partial_{x}\right) \phi=\hat{F}^{2}\left(\partial_{x}\right) \phi
$$


where $F(k)=-\mathrm{i} \hat{F}(\mathrm{i} k)$ is the dispersion function. (The ordinary wave equation is the case $F(k)=k$.) The right-hand side of (79) is defined by Fourier-transforming in $x$; it is thus a highly coordinate-dependent quantity, since defining it depends on integrating over the $x=$ const curves. It can be written somewhat more invariantly as

$$
\nabla^{2} \phi=\left(\hat{F}^{2}(X)-X^{2}\right) \phi
$$

where $X=\partial_{x}$. This makes clear that (as far as the propagation goes) the local Lorentz symmetry is broken by the choice of the vector field $X$, and nothing more.

Various choices have been investigated for the dispersion relation. The simplest one is the hyperbolic tangent form

$$
F_{\text {hyp }}(k)=k_{0} \tanh \left(k / k_{0}\right) \text {, }
$$

which has $F_{\text {hyp }}(k) \simeq k$ for $|k| \lesssim k_{0}$ and $F_{\text {hyp }}(k) \simeq k_{0} \operatorname{sgn}(k)$ for $|k| \gtrsim k_{0}$. This is the one we shall consider, except for a few comments. The effect of the dispersion relation is to replace the partial derivative $\partial_{x}^{2}$ at a single event by a weighted average over $x$-distances of coordinate size $\sim k_{0}^{-1}$.

A rough approximation to the propagation can be gotten by WKB methods. Approximate solutions are then of the form

$$
\phi \sim \exp \mathrm{i}(\omega t+k(x) x)
$$

where

$$
(\omega-k v(x))^{2}=F^{2}(k) .
$$

Consider either branch

$$
\omega-k v(x)= \pm F(k)
$$

of this, for fixed $x$ and $\omega$, as an equality between functions of $k$. The left-hand side is a straight line, but the right-hand side, for non-trivial dispersion, is a curve. There will thus be several points of intersection, typically three three on the positive branch and one on the negative, in the models considered. It is the extra solutions on the positive branch which allow the Unruh-type behavior.

Fix $\omega$, and imagine a wave-packet which, in the distant future consists of the spatial mode of moderate wave-number, $k \sim \omega /(1+v)$. As this is propagated back towards the past, it moves inwards towards smaller coordinate values, and $v(x)$ decreases towards -1 . This means that the slope of the line $\omega-k v(x)$ increases, and the line becomes more nearly tangent to $F(k)$. This gives a chance for the solution to develop a term corresponding to the right-most intersection point, and indeed Unruh has given an argument that at this point the packet turns around (its group velocity is zero at the point of tangency) and moves outwards at the group velocity defined by the right-most point. 
In fact, there is another feature that occurs simultaneously, as pointed out by Corley and Jacobson, "mode conversion:" the left-most mode is excited as well. ${ }^{23}$ It too turns out to contribute to the wave packet moving away (as we go backwards in time) from the hole.

The upshot of the analysis so far is that the late-time mode we started with, which was approximately that of a field satisfying the ordinary wave equation, has been propagated back in time to a wave packet moving away from the hole with group speed a bit slower than that of light. The packet involved consists of wave-numbers corresponding to the two extreme solutions of the dispersion relation, rather than the intermediate one in late time with which we started. By the time the modes have gotten to this point, their propagation is quite different from what one would have for the ordinary wave equation.

In order to outline the significance of this for particle creation, we must discuss the quantization of the theory.

\subsubsection{Quantization.}

We recall that particles are defined in terms of the decomposition of a field into positive and negative frequencies. In a general curved space-time, this decomposition is frame-dependent, and so is the notion of particles. However, if there is in a region of interest a naturally-determined frame, we have a natural candidate for the definitions of particles.

The particle content of the out-state is supposed to be analyzed in the asymptotically Minkowskian region $x \rightarrow+\infty$, and here there is a good definition of the positive-negative frequency decomposition. ${ }^{24}$ However, this is not the way the particle content of the in-state is defined. (Had we used the same definition for the in-state, we should find no particle production.) Rather, the particle-content of the in-state is determined by Fourier-analyzing with respect to time along the trajectories of the preferred class of freely falling observers, that is, along the curves $\mathrm{d} x=v \mathrm{~d} t$.

The motivation for this may be described as the hypothesis that the in-state should be vacuum as measured by these observers.

It has been verified numerically that these definitions of in-vacuum and outparticle content do result in a (nearly) thermal spectrum at the model's analog of the Hawking temperature. Just why this occurs is not really understood, however.

\subsection{The model of Brout, Massar, Parentani and Spindel}

These authors investigated a very similar situation to that of Corley and Jacobson above ${ }^{25}$ with hyperbolic-tangent dispersion, but with the after-collapse portion of the space-time glued onto an idealized collapse portion. Specifically, their model consists

23 Possibly this can be understood in terms of Stokes's phenomenon.

24 Although this requires a little care to define, given the dispersive character of the propagation.

25 And reached many similar conclusions, which will not be recapitulated here. 
of an early Minkowski-space region, followed by collapse of a pulse of incoming null matter to form a black hole, and then an Unruh-type region.

I shall not go into any of the technical analysis of this model (which follows the same spirit as that given above), but list two contributions that it makes:

In this model, the definition of the in-state is given by evolving from the Minkowski vacuum. (Recall that in the Corley-Jacobson model this depended on the freely-falling observers in the distant past.) Since the two models produce similar results, this is evidence that the Unruh-type models might not be artificially sensitive to the choice of in-state.

Brout et al. gave a WKB treatment, and were able (within the accuracy of the calculation) to give an analytic argument for the emergence of thermal radiation in this model. This must be counted as an important result. Subsequent refined and somewhat generalized treatments were given by Himemoto and Tanaka (2000) and Saida and Sakagami (2000).

\subsection{Summary and discussion}

Jacobson's (1993) paper effectively gave hope that one might circumvent the transPlanckian problem by exploiting its localization to the event horizon. The paper showed that if somehow an effective boundary condition near the horizon could result from (unspecified) deeper physics, Hawking's predictions could be recovered without trans-Planckian modes.

The Unruh-type models very remarkably reproduce results quite close to Hawking's. Although superficially they involve only a small tinkering with the field equation, in fact there is another key input, that is, the definition of the in-vacuum, which does not follow the standard one but is at least on its face a coordinatedependent concept. That the same results are produced in the Corley-Jacobson and BMPS models is evidence that this dependence is not too severe.

The modification of the field equation, that is, the dispersive propagation, is regular at the horizon. It is defined by modification of the spatial portion of the wave equation along a vector field $X=\partial_{x}$ which is perfectly regular and space-like at the horizon. One should note that the stationarity of this modification is used very strongly, and most essentially in the neighborhood of the horizon.

While these models do substantially reproduce the final radiation from black holes, the physical origin of that radiation is quite different. Instead of coming from the propagation of vacuum fluctuations through the collapsing space-time and out to infinity, the Unruh-type models only consider that portion of space-time after the collapse phase; the radiation arises from a hypothesized character of the quantum in-state.

In the Corley-Jacobson model, the character of the in-state is set by a preferred family of freely falling observers. They are not observers in "the distant past," since the entire analysis takes place in the portion of the space-time after the black hole 
has formed. In the BMPS model, the in-state is determined more invariantly, by propagation from an initially Minkowskian regime.

To what degree do these models resolve the trans-Planckian problem? There is some debate over this, partly on account of questions of how essential elements of the models are, and partly on account questions of the significance of trans-Planckian wave-numbers versus trans-Planckian frequencies.

The model with hyperbolic-tangent dispersion relation requires the use of unboundedly high wavenumbers as we follow the wave-packet back further into the past. ${ }^{26}$ One would like to know whether these are really essential, or whether, by settling for only a finite propagation backwards, one could avoid them. There is no definitive answer to this at present, but another result in the Corley-Jacobson paper suggests that the ultra-high wavenumbers are essential. Corley and Jacobson investigated a quartic dispersion relation, with bounded frequencies and bounded wavenumbers, but were unable to find a completely satisfactory construction of the theory in that case.

While the elimination of trans-Planckian frequencies is arguably progress, the presence of trans-Planckian wavenumbers is still disturbing. It means that one is (at least implicitly) invoking a continuum model of space on arbitrarily fine scales, something which one should at least be hesitant about. Also, the statement that trans-Planckian energies have been eliminated is significantly frame-dependent (if trans-Planckian wavenumbers have not been eliminated). For example, if $\omega \mathrm{d} t+k \mathrm{~d} x$ is a wave covector with $\omega$ cis-Planckian but $k$ trans-Planckian, then an observer with tangent $\cosh \xi \partial_{t}+\sinh \xi \partial_{x}$ will see a frequency $\omega \cosh \xi+k \sinh \xi$, which will be trans-Planckian even for moderate $\xi$.

The most important issue to be resolved within these Unruh-type models is the question of why the spectrum they produce is nearly thermal and (generalizing that) understanding how nearly the corresponding Bogoliubov coefficients match those of Hawking's predictions. (We have numerical and analytical arguments, but it seems there ought to be a brief basic physical argument.) When we do this, we may hope to have a better understanding of how sensitive the Unruh-type models are to: (a) the choice of coordinates; (b) the definition of the in-vacuum.

Finally, it should be noted that the possibility of dispersive propagation of highfrequency modes has been an important theme not just in work on Hawking's model, but in quantum gravity generally. See Himemoto and Tanaka (2000), AmelinoCamelia (2000), Błaut et al (2001); and Amelino et al (1998) for the possibility of experimental verification of such propagation.

\section{9. 't Hooft's $S$-matrix black hole theory}

It was 't Hooft who coined the term "trans-Planckian." Dissatisfied with the

${ }^{26}$ This issue does not come up in Unruh's original model, on account of the periodic boundary conditions he used. 
appearance of ultra-high frequencies and with some other aspects of the Hawking model, he pursued a program to develop an alternative theory.

In fact, 't Hooft $(1985,1993,1996,1997,1999)$ has put forward a host of interesting ideas, both in developing his theory and in elaborating the concerns which motivated it. Behind this variety of ideas is a set of intuitions about what should be the guiding principles in developing a fundamental theory: the ideas all aim to contribute to a unified cohesive view of the quantum character of black holes, and of space-time more generally. In important respects, this view differs radically (if sometimes subtly) from the conventional one.

One should have two, distinct, questions in mind when evaluating contributions like these. The first is rather severe: to what degree does the program as a unit really give a convincing treatment of the quantum character of a black hole? The second: what light do the individual ideas 't Hooft has introduced throw on the problem of understanding this quantum character?

Given the current state of the program (and the profoundly difficult problems it attacks), it would be premature to hold it to any very stringent standard as far as the first question goes. As to the second question, the program has already proved its value. It has given models for ways in which quantum-gravitational effects might enter essentially in the Hawking process, it has provided new physical perspectives on ideas from string theory, and, perhaps most importantly, it has provided a vivid reminder that there may be profound modifications to conventional quantum field theory in the presence of black holes.

While the goal of the program is a full quantum treatment (including quantumgravitational considerations) of black holes, this has not yet been achieved. And it is not yet really clear the extent to which the program can be said to support the assertion that black holes radiate. In its present stage of development, the program assumes that only rather slight modifications of the Hawking model, insofar as the radiation it predicts, are necessary.

It is not really possible to discuss the program's treatment of the Hawking process without describing the program as a whole. And to do this will require touching on some interesting aspects of Hawking's predictions (loss of quantum coherence, eventual explosion of black holes) which could themselves be discussed at length. However, only enough of a treatment of these for considering 't Hooft's program will be given.

I shall give an overview of the program, omitting the computations but focusing

on the ideas involved. I shall along the way comment on the open issues; however, it will be evident that many of these could be far more fully discussed than space here allows. Unless otherwise noted, all developments of the program discussed here can be found in the long review paper 't Hooft (1996).

\subsection{Overview of the program}

The point of departure for the program is the idea that black holes should constitute a species of particle. These particles may have internal degrees of freedom, 
and so they may exist in various quantum states. The first question to be asked about this particle species is, What is its spectrum? In particular, if the particle is placed in a box, is the spectrum continuous or discrete?

't Hooft argues that Hawking's theory would lead to a continuous spectrum. (This reasoning will be sketched below.) This in turn, it is argued, would make it impossible for black holes to evaporate completely; there would always be remnants. This is regarded as unacceptable, because no other particle species behaves in this way. It also raises the question of how virtual black-hole remnants might modify other aspects of quantum theory.

As 't Hooft himself points out, this argument rests on a number of assumptions which are debatable. Is it really legitimate to apply conventional quantum physics at the Planck scale, as needed to make assertions about remnants? Can we really talk about stationary states (eigenstates) of a Hamiltonian for black holes, since blackhole space-times contain not only an exterior stationary region but an interior timedependent region? Is it really legitimate, to the level of accuracy required, to talk about putting a black hole in a perfectly reflecting box? Is it legitimate to regard the box itself as fixed (infinitely massive)? How much trouble would virtual blackhole remnants really cause? If black holes can only form from fairly massive objects in the first place, couldn't it be that virtual remnants were always accompanied by higher-energy virtual excitations, and so suppressed?

Another concern which motivates 't Hooft is the apparent loss of quantum coherence associated with the Hawking process. That is, the Hawking process appears to convert an initially pure state (the in-vacuum or other in-state) to a mixed state (the quasi-thermal radiative state). As 't Hooft himself recognizes, it is far from clear that there really is any loss of coherence in the Hawking process. Accepting the conventional quantum-field-theoretic view (and leaving aside essentially quantumgravitational questions about what happens at the very end of the black hole's life), the mixing arises simply because one is neglecting the modes inside the hole. Yet 't Hooft is dissatisfied with this conventional picture, because it is the infinitely many modes within the hole which are responsible for the continuum of black-hole states.

\subsubsection{The spectrum.}

As mentioned above, one of the main goals of 't Hooft's program is to derive the spectrum of excitations of the black hole. This is done by assuming that Hawking's model is essentially correct, and appealing to time-reversal invariance. The use of time-reversal invariance here is, as we shall see, at least questionable.

To derive the density of states (up to a multiplicative factor), he compares the Hawking emission probability with the capture cross-section. This argument rests on time-reversal invariance (or more properly, $C P T$ invariance). There is some concern about this. The two amplitudes that must be related are

$$
\mathcal{T}_{\text {in }}={ }_{\mathrm{BH}}\left\langle M+E / c^{2}|| M\right\rangle_{\mathrm{BH}}|E\rangle_{\text {in }}
$$


and

$$
\mathcal{T}_{\text {out }}={ }_{\mathrm{BH}}\left\langle\left. M\right|_{\text {out }}\left\langle E|| M+E / c^{2}\right\rangle_{\mathrm{BH}},\right.
$$

where $|E\rangle$ represents a quantum of energy $E$, and "BH" of course stands for "black hole." The concern is that the time-reverse of a black hole is not a black hole, but a white hole. While the black hole, once it forms, is approximately static in some sense, it is not clear that this sense is strong enough to justify the use of timereversal invariance, since the Hawking process depends on the global history, before the quasistatic regime, of the space-time.

As emphasized by 't Hooft (see also Bekenstein 1972a,b), it is difficult to reconcile the Hawking model as it stands with any finite density of states. (That is, the multiplicative factor undetermined in 't Hooft's calculation might be infinite.) If, for example, one drops baryons into a hole, and lets it radiate, one can increase the baryon number indefinitely without increasing the mass. Thus either baryon number must cease to be a quantum number in a theory with black holes, or the theory must contain an infinite density of energy levels. Thus in order to achieve the discrete spectrum desired, some sort of modification of Hawking's theory is necessary.

\subsubsection{The holographic principle.}

Partly to render the density of states finite, and partly to deal with concerns about quantum coherence, 't Hooft (1993) made the radical suggestion that conventional quantum field theory seriously overcounted the internal degrees of freedom of a black hole. He proposed that there should be a holographic principle, which should relate these degrees of freedom to the boundary. He was led in fact to conjecture that such principles applied still more generally (to cases other than black holes); there have since been many attempts to formulate "holographic principles."

I shall make no attempt to discuss alternatives beyond those necessary to give a sense of 't Hooft's program; see Smolin (2001), Bousso (2002) for fuller accounts and reviews. For our purposes, it will be convenient to distinguish weak from strong forms, as well as those which are meant to apply to black holes from those which are meant to apply generally. (This terminology is not standard, although the weak/strong distinction is similar to one used by Smolin .)

Let $\Sigma$ be an acausal hypersurface ${ }^{27}$ with boundary $\partial \Sigma$ of area $A$. The black-hole case will be when $\Sigma$ lies in the interior of the hole and $\partial \Sigma$ on the event horizon; the general case will be when $\Sigma$ is unrestricted.

The weak holographic principle holds that the information in $\Sigma$ should amount to no more than $A /\left(4 l_{\mathrm{Pl}}^{2}\right)$ bits.

The strong holographic principle holds that the information in $\Sigma$ is actually contained in (or can be recovered from) at most $A /\left(4 l_{\mathrm{Pl}}^{2}\right)$ bits of information on the boundary.

\footnotetext{
27 An acausal hypersurface is one for which there is no causal path connecting two points. The condition is somewhat stronger than requiring it to be locally spacelike. For our purposes, one could equally well require $\Sigma$ to be achronal, that is, to have no points connected by timelike paths.
} 
The term "information" is of course vague; an important issue is how to make it precise. However, we shall accept it at an intuitive level.

A general holographic principle, even a weak one, would be very exciting, for it would imply non-local constraints on quantum field theories even in Minkowski space. This is because the number of field modes in a given volume would be constrained; presumably, there would be ultraviolet cut-offs arising from non-local physics. Depending on the scales and the circumstances in which these cut-offs became important, there could be profound alterations in physics. One should in particular keep this in mind for cosmological models.

While there have been attempts to formulate rigorous versions of general holographic principles, no really definitive one exists, and there is a serious concern about whether such a formulation will be possible. Consider a finite perturbation of the sphere $t=0, r=r_{0}$ Minkowski space to $t=f(\theta, \phi), r=r_{0}$. The area of the perturbed sphere is

$$
A=\int \sqrt{1-r_{0}^{-2}\|\nabla f\|^{2}} r_{0}^{2} \sin \theta \mathrm{d} \theta \mathrm{d} \phi
$$

where $\|\nabla f\|$ is the ordinary two-sphere norm of the gradient of $f$. By choosing $f$ small but oscillatory one can arrange for the area (87) to be arbitrarily close to zero while keeping $|f|$ arbitrarily close to zero. Thus there are arbitrarily small perturbations of the sphere making its area arbitrarily small. (Had we perturbed in space-like directions, we could have made the area arbitrarily large.)

This lack of stability of the area is especially troubling from a physical point of view. One should not have to specify to arbitrary precision the surface $\partial \Sigma$ in order to say how much information it surrounds. It is very difficult to see how, in such circumstances, one can take $A /\left(4 l_{\mathrm{Pl}}^{2}\right)$ as a measure of the information in $\Sigma$. (See however Bousso 2002 for other forms of general holographic principles which many workers consider more likely to be viable.)

For black holes, the area of the section $\partial \Sigma$ of the event horizon has a stability not enjoyed by the general case, and so the sort of pathology uncovered above cannot occur. We shall from now on consider only the black-hole case.

While it would seem that even the weak form of the black-hole holographic principle would meet 't Hooft's concerns about a continuum of black-hole states, in fact 't Hooft seems to seek a theory in which the strong form holds. He is not alone in this; many workers do not like the idea that information can actually be lost to a black hole, and would prefer some structure where the information can be recovered from the boundary of the hole.

It is rather difficult to see how the strong form of the black-hole holographic principle could be compatible with any classical description of physics within the hole, since it seems to be at odds with the principle of freely specifying Cauchy data on the interior of $\Sigma$. (Note that this point applies even when we take into account limitations in precision of specification of the data.) Indeed, attempts to explicate the physics 
of this strong form have led to the notion of black hole complementarity ('t Hooft 1993, Susskind et al 1993), which asserts that quantum measurements within the hole interfere with those at late times outside of it, in a manner contrary to that required by a classical causal structure of the hole. (In section 9.1.4, we shall see that 't Hooft is led to explicitly quantize the causal structure.) This is in accord with the "no quantum photocopy machine" principle: if at the quantum level the information contained in the infalling matter is recoverable from the boundary, it cannot be encoded in the interior.

While highly speculative, and apparently at odds with classical general relativity, the notion of black hole complementarity deserves serious thought. Since both black holes (through their causal properties) and quantum theory (through complementarity and measurements) profoundly affect the transmission of information, it is worth considering the possibility that they modify each other. Not just phenomena, but the laws of physics may be deeply altered in the presence of black holes.

To summarize: General holographic principles, that is, ones extending beyond the black-hole case, would potentially be of far-reaching importance, but it is not yet clear that they can even be stably formulated. If the strong black-hole holographic principle applies, as envisioned in 't Hooft's treatment, the interior of the hole seems to acquire an essentially quantum structure and may not even be approximable by a classical theory.

\subsubsection{The trans-Planckian problem.}

There is not yet a clear resolution of the trans-Planckian problem within 't Hooft's theory, although there have been some interesting suggestions.

The brick wall model corresponds to quantizing the field in a regime $r \geq(1+\epsilon) R_{\mathrm{Sch}}$, where $\epsilon$ is chosen large enough that no trans-Planckian modes appear. It is thus quite similar to Jacobson's (1993) timelike boundary condition (section 8.1). It should be noted that the actual quantization used by 't Hooft corresponds not to the Hawking model, but to the Boulware (1975) vacuum (a time-symmetric state defined in the exterior region only of the Schwarzschild black hole). As in the case of Jacobson's timelike boundary, the brick wall represents an ad-hoc model and is not meant to be a final theory.

It has also been suggested that the gravitational interactions of quanta in the vicinity of the horizon are strong enough to modify the theory and provide an effective "brick wall" which serves as a trans-Planckian cut-off. One might say the quanta are supposed to build their own brick wall. This idea, as presently conceived, seems to require a radical alteration of the conventional Hawking picture. This alteration appears in a model calculation as a large, unconventional stress-tensor near the horizon ('t Hooft 1997). An appeal to black-hole complementarity is made to justify this stress-energy.

It is possible to give a more direct understanding of the issue. First note that, in the conventional picture, the excitations that appear to distant observers as Hawking 
quanta do not appear as excitations at all near the horizon, but as combinations of vacuum fluctuations. This is simply because the Hawking process after all converts any state which is Hadamard at the horizon to thermal radiation. As one follows a Hawking quantum backwards in time, there comes a point where the curved-space difficulties in defining what a "particle" is become significant, and the quantum would look to observers not like a single particle, but like a more complicated field excitation. On dimensional grounds, this must happen for $r \sim 3 G M / c^{2}$. Following the quantum backwards much further, it must become simply a combination of vacuum fluctuations.

The proposal that gravitationally-interacting quanta should "build their own brick wall" requires energetic real quanta in the neighborhood of the horizon. If these are to be the Hawking quanta, then one must modify the conventional theory so that the quanta are real particles near the horizon. The modified stress-tensor suggested in 't Hooft (1997) can be viewed as the result of just such a change.

On the other hand, it should be noted that up to this point the discussion has left aside what actually happens when quantum measurements of Hawking quanta are made. This neglect is customary in analyses of the Hawking effect but is not really justified. When a measurement of Hawking quanta is made, the state vector is reduced, and near the horizon this does result in a complicated excitation of ultra-high frequency modes. However, the fact that the notion of what a "particle" is alters as one goes from a neighborhood of $\mathcal{I}^{+}$to a neighborhood of the horizon means that this excitation is not simply the presence of a high-energy quantum. These points will be discussed more fully in Helfer (2003).

\subsubsection{The S-matrix theory.}

One of the goals of 't Hooft's program is a theory which explicitly preserves purity. As we saw above, the apparent destruction of purity comes about in the conventional picture by ignoring the internal black-hole modes. In 't Hooft's theory, it is anticipated that this picture will be modified by the strong form of the holographic principle; the details of this have yet to be worked out. However, 't Hooft develops the theory by assuming that it can be written in $S$-matrix form, and then deducing properties of the $S$-matrix.

The main idea may be outlined as follows. In order to avoid difficulties with bound states, it is assumed that the in- and out-states contain only asymptotically free particles. Thus a fiducial in-state $\left|\mathrm{in}_{0}\right\rangle$ might look, in the distant past, like a set of particles whose trajectories will lead them eventually to implode and form a black hole. A fiducial out-state $\mid$ out $\left._{0}\right\rangle$ would contain all the particles emitted after the final evaporation of the black hole via Hawking radiation. Note that this implicitly involves quantum-gravitational assumptions, since it necessarily involves the Planckscale physics in the very last, explosive, stage of the evaporation.

Evidently, actually developing the theory in this form would require studying the black hole over its entire existence, and also addressing difficult quantum-gravitational issues. This would be too hard, at least initially, and what has been done so far 
corresponds rather to studying the Hawking process over periods of time in which at least up to quantum corrections the black hole can be taken to be static.

The idea is to start from some (unknown, but finite) amplitude $\left\langle\right.$ out $\left._{0} \mid \mathrm{in}_{0}\right\rangle$ and perturb both the in- and the out-states by adding or deleting particles from them in order to work out an $S$-matrix (up to a phase, presumed irrelevant). In practice, this is done by assuming the matrix (operator, really) factors as

$$
S=S_{\text {out }} S_{\text {hor }} S_{\text {in }}
$$

where $S_{\text {in }}$ represents the propagation of particles inwards from infinity towards the horizon, the factor $S_{\text {hor }}$ is the scattering near the horizon, and $S_{\text {out }}$ represents propagation outwards from the horizon to infinity.

Of course, the factorization (88) can at best only be expected to be approximate and only to apply to one sector of the theory. Making these limitations precise is highly non-trivial, and is related to some deep questions, to be taken up below, about whether any of these factors should in fact be expected to be unitary.

Each of the three factors should ultimately be important to 't Hooft's program. The factor $S_{\text {in }}$ contains the information of the collapsing matter, and so presumably much of the treatment of the quantum-coherence issues should be bound up with understanding it. However, some ingoing particles can be considered in the factor $S_{\text {hor }}$, and so one can get a sort of perturbative handle on the issue by studying $S_{\text {hor }}$. Finally, a key step in the Hawking process is the propagation of field modes outwards from a neighborhood of the horizon, accomplished here by $S_{\text {out }}$.

Of the three factors, the program has so far been concerned with the middle one, $S_{\text {hor }}$ 't Hooft considers the addition or deletion of a number of particles near the horizon, and the way that an incoming particle might distort the gravitational field and so alter the trajectory of an outgoing particle. ${ }^{28} \mathrm{He}$ is able to deduce a great deal of the form of $S_{\text {hor }}$ based on this physics. This is of considerable interest as a model of possible quantum-gravitational corrections.

The way in which the computation of $S_{\text {hor }}$ really speaks to the physics of the Hawking process needs to be elaborated. As discussed above, the Hawking quanta arise, not from ordinary particles near the horizon, but from combinations of highfrequency vacuum fluctuations there. These, when propagated outwards, give rise to the observed Hawking particles. On dimensional grounds, one would expect the concept of a Hawking quantum as a quantum-mechanical particle to become valid for $r \gtrsim 3 G M / c^{2}$; much closer to $r=R_{\text {Sch }}$, it should be a combination of vacuum fluctuations.

Whether the Hawking quanta appear as particles of as combinations of vacuum fluctuations (or as some intermediate) to $S_{\text {hor }}$ depends then on at what distance from the horizon the transition from $S_{\text {hor }}$ to $S_{\text {out }}$ is made. The analysis which has been

${ }^{28}$ In this computation, the particles are approximated as pointlike; more properly, their finite Compton wavelengths are neglected. 
made in 't Hooft's program presumes the Aichelburg-Sexl metric form is valid, which essentially means one is much closer to the horizon than to $r=3 G M / c^{2}$. Thus it seems that the transition must be made close to $r=R_{\text {Sch }}$, and this suggests that the Hawking quanta are actually created, at least within the conventional picture, by the factor $S_{\text {out }}$. It would simply be the Hadamard form of the state in the $S_{\text {hor }}$-sector which is the seed for their creation.

To resolve this concern about exactly how $S_{\text {hor }}$ contributes to the Hawking process, one would need a fuller treatment of it together with $S_{\text {out }}$, keeping careful track of the precise hypotheses on the transition from one regime to the other. Alternatively, perhaps the sorts of modifications to Hawking's theory made in the "quanta building their own brick wall" model discussed above might be invoked.

One final, important, aspect of 't Hooft's $S$-matrix computation should be brought out. As mentioned above, the computation explicitly takes into account the distortion of space-time geometry caused by incoming particles. This led 't Hooft to introduce the idea of quantum operators representing the location of past and future horizons, and to start to consider the consequences of quantum complementarity for measurements of these operators. While these ideas are very speculative, the profound importance of addressing such issues - how quantum behavior might alter the global causal structure of space-time - should be clear.

\subsubsection{Purity and unitarity.}

A major goal of 't Hooft's program is a theory which is unitary. This is motivated partly by a desire to make black holes look just like any other quantum-mechanical particles, and partly by a desire to preserve purity of quantum states. While it is possible that this will be achieved, there are subtleties in understanding just what this would involve. While unitarity would imply the preservation of purity, the converse need not be true, and there are questions about whether (or to what approximation) a unitary theory can be constructed even for the factored $S$-matrix approach.

We shall adopt the conventional Heisenberg picture, so that the state vector remains fixed (except when reductions occur) and the fields are functions (operatorvalued distributions, really, on space-time). As long as this picture is applicable, preservation of purity is automatic. Let us turn to unitarity.

In ordinary quantum mechanics, the evolution of a set of canonical variables $\left(p_{j}, q_{j}\right)$ from one time to another must be unitarily implementable, that is, there must be a unitary $U$ such that

$$
U\left[\begin{array}{c}
p_{j} \\
q_{j}
\end{array}\right]_{t_{0}} U^{-1}=\left[\begin{array}{c}
p_{j} \\
q_{j}
\end{array}\right]_{t_{1}} .
$$

This is a consequence of the Stone-von Neumann theorem: for finitely many degrees of freedom, as long as the canonical commutation relations are preserved, such a unitary $U$ must exist.

However, for field theories, which have infinitely many degrees of freedom, the situation is more complicated. Evolution does preserve the canonical commutation 
relations, but it need not be unitarily implementable. Indeed, if the evolution is represented as a Bogoliuobov transformation, there is a well-known squaresummability criterion for unitary implementability (Wald 1994).

In certain restricted cases, one can argue on physical grounds that one expects unitarily implementable evolution. For example, for any Poincaré-invariant quantum field theory, the evolution from a $t=$ const to a $t^{\prime}=$ const surface (with $t$ and $t^{\prime}$ inertial time coordinates) should be given by a Poincaré motion, which one would expect to be represented by a unitary operator. (Indeed, this is almost a definition of what one would mean by a Poincaré-invariant quantum field theory.)

It is something of a surprise to find out, though, that unitary implementability is the exception rather than the rule. In Helfer (1996), for quantum fields propagating through a general curved space-time, it was shown that the "sum of squares" to be computed in the Bogoliubov criterion is an integral of products of Green's functions and two-point functions. For an explicit non-pathological class of cases this was computed and found to contain ultraviolet divergences. These divergences will also be present generically, on account of the smooth dependence of the Green's and two-point functions on the structure.

Physically, these divergences come about because the two-point functions have different asymptotics on different Cauchy surfaces. As mentioned earlier, reasonable physical states are Hadamard, that is, their ultraviolet two-point asymptotics are modeled on $-(4 \pi)^{-1}(p-q-\mathrm{i} \epsilon)^{-2}$ in Minkowski space. Now in curved space-time, this asymptotic form is of course expressed in terms of the metric distance between two events $p$ and $q$. In particular, then, the two-point function restricted to a Cauchy surface encodes the surface's intrinsic metric. That is, the geometry of the Cauchy surface can actually be recovered from the ultraviolet asymptotics of the two-point function. This means that if the two Cauchy surfaces are not isometric, the two-point functions on them will have different ultraviolet asymptotics. Finally, the "sum of squares" in the Bogoliubov condition is essentially an integral over the square of the difference of the two-point function between the two surfaces (times a wave-vector space integral). Since in general two Cauchy surfaces are not isometric, this difference cannot be made to vanish. And it is found that the integral in question diverges generically.

Another way of looking at this result is that the two-point function is a correlation function between vacuum fluctuations. Since the ultraviolet asymptotics of this correlation function, restricted to a Cauchy surface, allow one to recover the geometry of the surface, the physics represented by this ultraviolet asymptotic regime is essentially different on one surface from another (unless they are isometric). This is why the evolution is not unitarily implementable except in exceptional cases.

It should be noted that the fact that one has a clear-cut way of isolating the divergences means that one could introduce a cut-off; the cut-off theory would be unitarily implementable. This also means that the theory is formally unitarily implementable; the difficulty is in taking the cut-off to infinity. 
This issue of non-unitarity is not really fully understood. However, there are some hints of how to resolve it.

First, while the non-unitarities are most evident in the case of quantum fields in curved space-time, they also occur (but with lower divergences) for quantum fields propagating through general time-dependent external potentials in Minkowski space (Helfer 1996). ${ }^{29}$ We shall consider charged quantum fields moving in a time-dependent external electromagnetic potential.

For these charged fields, there is a reasonable, conservative speculation as to what the resolution of the non-unitarity problem is. This is that the non-unitarities result from the neglect of quantum fluctuations in the electromagnetic field, and a full quantum-electrodynamic treatment (if this could be really achieved) would indeed be unitary.

If a similar resolution holds for gravitational fields, then it would appear a quantization of gravity would be necessary to restore unitarity. On the other hand, it might be that quantum gravity cannot be understood without a deeper treatment of the physics of reduction (see e.g. Penrose 1986); so perhaps a quantum gravity theory should not have unitarity as a goal.

In particular, the analysis above suggests that the factors $S_{\mathrm{in}}$, $S_{\mathrm{hor}}, S_{\text {out }}$ in 't Hooft's program cannot be exactly unitary without a quantization of gravity. Some such quantization is implicit in 't Hooft's program, and, as we saw, progress towards it has been made in the case of $S_{\text {hor }}$.

Finally, while a basic tenet of 't Hooft's program is that ultimately a unitary theory should be attainable, we have seen here that unitarity is a stronger condition than preservation of purity.

\subsection{Summary}

't Hooft has proposed a radical and ambitious program; to execute it successfully will require deep modifications of conventional quantum field theory, and the incorporation of essentially quantum-gravitational ideas. The program is still in a stage of development, and it is not yet clear that it will meet its goals. It does not at present provide a clear resolution of the trans-Planckian problem.

The program has however proved valuable in raising deep questions about the possibility of modifying quantum field theory, and also for providing models for possible such modifications and for quantum-gravitational effects. While these models may ultimately not turn out to be correct, innovative ideas such as these are of the greatest importance in tackling the very difficult problems involved.

\section{Evidence from theories of quantum gravity}

In this section, I shall review the evidence for the Hawking effect from theories of

29 The explanation given above in terms of intrinsic metrics does not apply, of course, but it turns out that the subdominant ultraviolet asymptotics encode the external field. 
quantum gravity. Of course, all of this work is speculative, but it is very important in trying to understand what might happen beyond the classical treatment.

Virtually all work discussed here is quite technical, and no attempt to present the details of arguments will be given; the emphasis will be on the basic physical assumptions and the results.

\subsection{Dilatonic black holes}

These are meant to be model theories of the s-wave sector of quantum gravity coupled to matter fields. (Thus in particular the radius of any sphere of symmetry becomes an operator, and it is essentially this which is the "dilaton field.") They are non-linear field theories. Their forms are motivated by formally integrating out the angular dependence of Lagrangians which are supposed to represent the theories. This involves a number of conceptual and technical problems, most especially related to the diffeomorphism invariance. The results therefore are not unique, but in each case represent the workers' best opinions of how to resolve these difficulties. See Grumiller et al. (2002) for a recent review.

Dilaton models which are semiclassical perturbations of Schwarzschild (coupled to scalar matter) have been investigated and have been able to reproduce the Hawking radiation (Kummer and Vassilevich 1999), but the freedoms in defining the theories in light of our present ignorance are large enough that not all admissible theories do produce Hawking radiation. One can even have negative luminosities (Balbinot and Fabbri 1999).

Since conventional semiclassical quantum field theory in curved space-time does predict Hawking radiation, how can even some semiclassical dilaton models fail to predict it? One way of phrasing the answer is there are difficulties in ensuring that dimensional reduction and renormalizations (or well-definition) of the theory commute. The underlying physical question seems to be that even though one "integrates out" many degrees of freedom, the vacuum fluctuations from these degrees might leave a non-trivial modification of the semiclassical theory. Our limited understanding of this should serve as a caution of the need for confronting the subtleties involved a correct treatment of the physics.

\subsection{TTFKASS}

M-theory (the theory formerly known as superstrings) has been able to reproduce the Bekenstein-Hawking entropy formula $S=A /\left(4 l_{\mathrm{Pl}}^{2}\right)$ for "near-extremal" black holes (see Peet 2000 for a review). These are a restricted class of holes, with charge very nearly equal to their mass (and surface gravity very nearly zero).

The argument for this is a mode-counting one involving one of the remarkable dualities discovered in the theory. It is thus a serious candidate for an explanation of the origin of black-hole entropy (at least for near-extremal holes). (Recall that the Hawking process does not do this.) 
While the mode-counting result is clear, it is difficult to follow the details of the physics through the duality and understand what a black hole would really look like in this case. Would Hawking radiation be predicted only in collapse situations or for all black holes? What would be the resolution of the trans-Planckian problem? (The near extremality - that is, nearly zero $T_{\mathrm{H}}$ - makes understanding this harder.) These questions are, at present, unresolved.

\subsection{Ashtekar's approach}

Ashtekar's approach to quantum gravity, a Hamiltonian one based on his "new variables," is much more obviously connected to general relativity than is M-theory. Nevertheless, one still has both conceptually and technically difficult tasks in trying to model the Hawking process, even in idealized ways, in this theory.

Remarkably, however, workers using this approach were able to push through a mode-counting argument to reproduce the relation $S \propto A$ (although not to fix the constant of proportionality). See Ashtekar et al. (2000), and references therein. This result holds not just for extremal black holes, but for any spherically-symmetric hole large compared to the Planck scale.

It should be pointed out that in this approach there is a certain ad-hoc character to the treatment of the horizon. (This is a Hamiltonian approach, and so data must be given on a Cauchy surface. However, the location and even the existence of an event horizon are in general difficult to determine from Cauchy data, as they are defined in terms of the global asymptotics of the space-time. Thus a full treatment would require, at least implicitly, information about the global evolution of the data - clearly, such a treatment would be unrealistically difficult.)

One would hope it is possible to understand the resolution of the trans-Planckian problem within this approach, but this seems not yet to have been treated in detail. At the least, from the general nature of the construction, one would expect each Hawking quantum to become entangled with a quantum perturbation of the horizon.

\subsection{Euclidean quantum gravity}

Possibly the earliest quantum-gravitational approach to verifying the Hawking mechanism was undertaken within the Euclidean quantum gravity program. Gibbons and Hawking (1977) evaluated the partition function

$$
Z=\operatorname{tr} \exp -\beta H
$$

in the one-loop approximation, and were able to reproduce the Bekenstein-Hawking formula for the entropy.

There is a question of the consistency of this scheme, however, which also brings up a more general problem (Wald 1994). If black-hole entropy can indeed be interpreted as the logarithm of the number of available states, then the density of states must grow spectacularly quickly $\left(\sim \exp 4 \pi\left(M / m_{\mathrm{Pl}}\right)^{2}\right)$ with the mass of the hole. This makes it very hard for thermodynamic averages like (90) to converge. 


\section{Quantum character of space-time}

In the previous section, I discussed the consequences, for the Hawking analysis, of assuming particular theories of quantum gravity. But there is another approach to understanding quantum-gravitational effects, where rather than hypothesizing a particular theory of quantum gravity, one considers general properties of quantum theory and studies how they might be integrated with general relativity. While there is not a sharp line between the two approaches, the latter one does tend to approach questions more directly physically than mathematically. Given the highly speculative nature of any specific theory of quantum gravity, the complementary character of the second approach is especially valuable.

\subsection{Validity of the semi-classical approximation}

I will begin, not by describing specific possible quantum-gravitational issues, but by discussing a popular fallacious argument to the effect that one can neglect such concerns in modeling the Hawking process, except perhaps in accounting for the change of the hole's mass over very large times. I'll recount it, challenging the alert reader to spot the difficulty.

The argument goes like this. Everyone agrees that the Hawking effect is a very small perturbation of an essentially classical system, the gravitational collapse of a large body. So to first approximation, it ought to be perfectly legitimate to treat the quantum field as simply "painted on" to the classical background. Only over times for which the emission of Hawking quanta can significantly perturb the geometry of the collapsing object should this treatment break down.

Here is a parallel argument about another physical system. Consider the emission of a quantum of radiation by an atom embedded in a crystal. Since everyone agrees the crystal is essentially a macroscopic system, it ought to be sufficient to treat the crystal classically, and model the emitting atom quantum-mechanically. Only for very fine measurements of (for instance) the momentum of the crystal would it be necessary to go beyond this.

The argument about the crystal is an argument against the Mössbauer effect, and so is specious. It is correct insofar as it asserts that the back-reaction of the emission on the crystal only makes the tiniest correction to the macroscopic state of the crystal. It errs crucially in neglecting the fact that the coupling between the emitting atom and the crystal has a quantum character, and one can only get the physics right by taking into account the existence of quantized phonon modes in the crystal. That is, the coupling of the atom to the crystal constrains the allowed transitions of the atom; it restricts the allowable matrix elements for the transition of the atom. Neglecting the coupling, the atom may make transitions to a continuum of recoil momenta; taking the coupling into account, most of these transitions are forbidden. What forbids them is conservation of momentum, of the atom plus crystal system, at the level of quantum operators. Thus some quantum characteristics of the crystal enter essentially. 
While the analogy between the Hawking process and the Mössbauer effect cannot be made a very close one, the point being made here is simply that when a large system (the collapsing object; the crystal) couples to a small quantum one (the quantum field; the emitting atom), one needs to consider whether the coupling will lead to constraints on transitions.

\subsection{Quantization of black-hole area}

It is certainly credible that quantum gravity should act to quantize a black hole's area. Indeed, such a feature would attractively unite the interpretation of the black hole's area with entropy with the information-theoretic interpretation of entropy. So let us consider the consequences of such a relation. This argument follows work of Bekenstein and Mukhanov (1995), the importance of which was emphasized by Ashtekar (1998). (For a list of works on area quantization, see Das et al 2002.)

Let us suppose for simplicity that the black hole's area is quantized in units $\alpha l_{\mathrm{Pl}}^{2}$, where $\alpha$ is a numerical constant. Now, when the black hole emits a quantum of energy $\Delta E$, its area changes by $\Delta A \simeq 32 \pi\left(G^{2} / c^{6}\right) M \Delta E$, and so we must have

$$
n \alpha l_{\mathrm{Pl}}^{2}=32 \pi\left(G^{2} / c^{6}\right) M \Delta E,
$$

or

$$
\begin{aligned}
\Delta E & \simeq(32 \pi)^{-1} n \alpha\left(l_{\mathrm{Pl}}^{2} c^{6} / G^{2}\right) M^{-1} \\
& =n(\alpha / 4) k T_{\mathrm{H}} .
\end{aligned}
$$

In other words, the energies of the Hawking quanta would be discrete multiples of $(\alpha / 4) k T_{\mathrm{H}}$. If $\alpha$ were around unity, this would lead to a line spectrum, rather than a continuum. If $\alpha$ were much smaller than unity, the spectrum would be quasicontinuous. But if $\alpha$ were larger than about 10, almost all Hawking radiation would be suppressed, since there would be few Hawking quanta energetic enough to allow the requisite transition of black-hole states.

Any discretization of the spectrum will tend to increase its information-content and therefore to decrease its entropy. However, this does not affect the BekensteinHawking formula and thus one expects that some discretizations of the spectrum may lead to violations of the generalized second law. This has been confirmed by computations of Hod (2000).

Of course, one need not consider simply a quantization of the area in integral multiples of $\alpha l_{\mathrm{Pl}}^{2}$; there are other possibilities. The real point of the investigation, from our point of view, is to show that quantum-gravitational corrections which could very plausibly be present on dimensional grounds could considerably alter not just Hawking's predictions, but the conjectured connection of it with thermodynamics.

Finally, it should be noted that these sorts of concerns are beyond the transPlanckian problem. The trans-Planckian problem is a difficulty within the semiclassical approximation. Even if the trans-Planckian problem were to be resolved by some sort of (say) non-standard field propagation, there is no obvious way that such a resolution would speak to the consequences of quantizing black-hole area. 


\subsection{Quantum measurement issues}

We can get another perspective on the possible consequences of a quantum character of space-time, as follows.

Measurements of geometrical quantities in space-time are presumably subject to quantum limitations, just as measurements of other quantities are. This means that all of the calculations we do with classical general-relativistic quantities should be regarded as approximations valid for when quantum fluctuations are negligible. This approximation would break down when those fluctuations are significant, and we may try to estimate when this happens.

The key geometrical quantity that enters in the Hawking analysis is $v(u)$, the mapping of surfaces of constant phase. For example, the rough approximation to the propagation we used was simply

$$
\left.\phi_{0,0}^{0}(u)\right|_{\mathcal{I}^{+}}=-\left.\phi_{0,0}^{0}(v(u))\right|_{\mathcal{I}^{-}} .
$$

We are now going to admit the possibility that it may not be adequate to treat $v(u)$ as a classical quantity to arbitrary accuracy, that it might be subject to quantum fluctuations. This means that $\left.\phi_{0,0}^{0}(u)\right|_{\mathcal{I}^{+}}$entangles the state of the field at $\mathcal{I}^{-}$with $v(u)$. (Note that this is similar to 't Hooft's 1996 idea of quantizing the horizon, discussed in section 10.1.4.)

Let us consider a measurement of $v^{\prime}(u)$. (It is the quantities $v^{\prime}(u)$ and $v^{\prime \prime}(u) / v^{\prime}(u)$ which enter expectations of the field operators most directly.) The most direct way to measure $v^{\prime}$ would be to send in (massless, non-interacting) field quanta of known frequencies from $\mathcal{I}^{-}$and measure their frequencies at $\mathcal{I}^{+}$. The red-shift would be $v^{\prime}(u)$ (and ratios of successive differences of this would give an estimate for $v^{\prime \prime} / v^{\prime}$ ). It should be emphasized that these "probe" quanta have nothing direct to do with any Hawking quanta and could be of a different species than the Hawking quanta to be observed. The probe quanta are just part of a thought-experiment to understand what the effects of quantum limitations on accuracies of measurement might be.

For such a measurement to be relevant to an observation of Hawking quanta over a time interval $\Delta t$ (near $\mathcal{I}^{+}$), the probe quanta must have frequencies near $\mathcal{I}^{+}$of $\omega_{+} \gtrsim 1 / \Delta t$. However, this means that their frequencies near $\mathcal{I}^{-}$must have been $\omega_{-}=\left(v^{\prime}\right)^{-1} \omega_{+} \gtrsim\left(v^{\prime}\right)^{-1} / \Delta t$. Since $\left(v^{\prime}\right)^{-1}$ increases exponentially quickly, we quickly pass any finite scale for $\omega_{-}$. In particular, presumably it is impossible to have $\hbar \omega_{-}>E_{\mathrm{Pl}}$, and thus there is a fundamental limitation to the possible measurements. If we take $\Delta t \sim R_{\mathrm{Sch}} / c$ (the time scale for the emission of a Hawking quantum), then this fundamental limitation is reached when

$$
v^{\prime}(u) \sim m_{\mathrm{Pl}} / M
$$

This of course happens very quickly. It is in fact the same scale at which the transPlanckian problem sets in. 
It should be emphasized that the difficulties at this scale are potentially much more severe than simply an entanglement of the state of the field with the quantum state of space-time. The difficulty is that at this point trans-Planckian physics enters, and it is probably not meaningful to talk about the value of $v^{\prime}$ even as a quantum operator.

\section{Experimental prospects}

The real proof or disproof of Hawking's predicted radiation, of course, would be experimental. It is not impossible that we shall one day have experimental results one way or the other. The main difficulty is that, at present, there are no known black holes of the right size that we might observe Hawking radiation from them; also, there is the difficulty of knowing exactly what one should look for - one must go beyond Hawking's rather idealized linear-field model to have an accurate physical prediction of what a Hawking-radiating black hole would really look like.

\subsection{What sorts of black holes might exist?}

At present, two sorts of black holes are believed to have been observationally identified: ones of a few solar masses, and "super-massive" ones (with masses $\left.\gtrsim 10^{6} M_{\odot}\right)$. There seems to be no prospect for identifying Hawking radiation from such objects: they would be far too cold and far too dim.

It is very plausible that black holes might have been created in the early Universe, from density fluctuations (and also possible that they may have formed by other means). These are called primordial black holes. For our purposes one should note that "primordial" is not to be taken absolutely; these holes (if they exist) were formed by gravitational collapse after the big bang and should, according to Hawking's theory, radiate.

Estimates of the mass function of primordial black holes (number of black holes of a given mass) are model-dependent. The main constraints on the mass function come from the assumption that black holes would radiate, and that too much radiation would either be directly observed or disturb other features of the cosmological model. ${ }^{30}$ On the other hand, if black holes do not radiate, then the only known constraint is that they should not contribute more than the critical density. See Liddle and Green (1998) for a summary of constraints.

No generally-accepted models have been put forward for the formation of small (sub-solar mass) black holes later in the Universe. We should be mindful, though, that astronomy has a tradition of surprising us.

To summarize: If we do not assume that black holes radiate, there are few constraints on them in cosmological models.

30 Radiation from black holes can potentially disturb nucleosynthesis, alter the cosmic microwave background, and change the entropy-to-matter ratio. 


\subsection{What would a Hawking-radiating black hole really look like?}

Hawking's calculations apply directly only to massless linear fields. No such fields are believed to exist in Nature; all fields are believed to couple to others. How important is the assumption of linearity, and how would the inclusion of nonlinear corrections affect the predictions?

These are questions to which we do not have wholly convincing answers, partly due to technical difficulties in treating nonlinear fields, and partly due to the transPlanckian problem. In principle, one would have to work through the steps of Hawking's derivation, but for a realistic non-linear field theory, and this would not only be a massive quantum-field-theoretic undertaking, it would involve hypotheses about what physics is like in the ultrahigh energy regimes necessary to the Hawking process in the vicinity of the event horizon.

However, some progress has been made, along with some very nice physical analyses. Essentially the starting-point for these is the assumption that for (say) $r \gtrsim 10 R_{\text {Sch }}$ one can hope that Hawking's predictions would give rise to a conventional field theory with a central source at the Hawking temperature. General-relativistic corrections should be negligible, and one has a (difficult) conventional field-theory problem to solve.

In order to get an idea of the physics and the open questions, let us take the following, very crude, division of scales (figures are given to order-of-magnitude only):

$k T_{\mathrm{H}} \lesssim 1 \mathrm{MeV}$. (Energies below the threshold for electron-positron production.) In this case, one expects Hawking radiation of photons and neutrinos, modeled as free massless particles. The corresponding black-hole mass range is $\gtrsim 10^{17} \mathrm{~g}$.

$1 \mathrm{Mev} \lesssim k T_{\mathrm{H}} \lesssim 100 \mathrm{MeV}$. In this range, electron-positron production, as well as non-linear quantum-electrodynamic effects, must be considered, but strong-interaction physics can be neglected. The corresponding mass range is $10^{15} \mathrm{~g} \lesssim M \lesssim 10^{17} \mathrm{~g}$.

$100 \mathrm{MeV} \lesssim k T_{\mathrm{H}} \lesssim 1 \mathrm{GeV}$. In this range, one must take into account the production of unstable hadrons (mesons) which will decay into leptons and photons. The corresponding mass range is $10^{14} \mathrm{~g} \lesssim M \lesssim 10^{15} \mathrm{~g}$.

$1 \mathrm{GeV} \lesssim k T_{\mathrm{H}}$. This is the scale at which proton-antiproton production sets in.

The first one of these is fairly well understood, but beyond that there are questions. The appearance of the radiation depends crucially on precisely what sorts of self-interactions the Hawking quanta do undergo on their way out from the hole. For example, it has been suggested (Heckler 1997, Cline et al 1999) (although disputed: Kim et al 1999) that for $k T_{\mathrm{H}} \gtrsim 200 \mathrm{MeV}$ quantum-chromodynamic Bremsstrahlung and other effects become important enough for a photosphere to form. This would reduce the observed temperature. Indeed, any mechanism which resulted in (approximately) thermalizing the final radiation at a radius $r$ would reduce the temperature by $\sim\left(R_{\mathrm{Sch}} / r\right)^{2}$, since the luminosity goes as $T^{4} r^{2}$. 
While the regimes above only go down to around $10^{14} \mathrm{~g}$, this is only because that much suffices to give a general consideration of the sorts of physical questions that come up, and our (present) lack of ability to answer them. One should certainly consider the possibility of smaller (hence higher $k T_{\mathrm{H}}$ ) holes.

Thus there is significant uncertainty in what we should expect for the emergent spectrum for a Hawking-radiating black hole of given mass. This point needs to be considered, not only in asking what sorts of holes might be detected observationally, but in estimates of the effects of Hawking-radiating holes in the early Universe. Lowerthan-expected temperatures would presumably lead to smaller effects of black holes on cosmology, and therefore to weaker constraints of the possible number of black holes in the early Universe.

Whatever the self-interactions of the Hawking quanta are on their way out from the hole, total luminosity (including all species) should be given by Hawking's prediction (taking account of the number of effective degrees of freedom at any temperature). These luminosities are quite low, being

$$
L \simeq 10^{20} \mathrm{erg} \mathrm{s}^{-1}\left(10^{15} \mathrm{~g} / M\right) .
$$

For comparison, the Sun's luminosity is $\sim 4 \cdot 10^{33} \mathrm{erg} \mathrm{s}^{-1}$. Thus one needs a great many black holes, or ones close to the Earth, for the radiation to be detectable.

Given the uncertainties in the calculations, I will only quote two numerical estimates, to give the reader a sense of the magnitudes involved. Heckler (1997) estimates that a $10^{13} \mathrm{~g}$ black hole could only be detected as a point source by the GLAST satellite if it were closer than about $1.5 \cdot 10^{-3} \mathrm{pc} \simeq 30$ astronomical units. One can also infer limits on the density of evaporating black holes by requiring their radiation not exceed the observed gamma-ray spectrum. Halzen et al (1991) give such a limit

$$
\Omega_{\mathrm{pbh}} \lesssim 7.6( \pm 2.6) \cdot 10^{-9} h_{0}^{-1.95 \pm 0.15}
$$

where $h_{0}$ is the scaled Hubble parameter (of order unity). The reader is cautioned that both of these are model-dependent (and different models are used) and are displayed only to give a rough sense of the scales involved.

Work aimed at providing realistic models of what radiating would look like is a subject of active research, with much scope for interesting physics to be discovered. Besides the papers already mentioned (Cline et al 1999, Halzen et al 1991, Heckler 1997), see Daghigh and Kapusta (2002), Kapusta (2000). A brief summary from the point of view of experimental prospects occurs in section II.F of the Snowmass report (Buckley et al 2002).

\subsection{Summary}

To confirm the existence of Hawking radiation, we would need (not only observations), but reliable models of what radiating black holes would actually look 
like. We are at present limited in our understanding of how to construct such models, as they require the study of non-linear, realistic, quantum field theory at high temperatures. This is a very difficult technical problem, but it does not require the resolution of foundational issues.

To show that black holes did not radiate, we would need to detect the holes, or to have strong indirect evidence of their existence, as well as to show that they do not radiate. At present we are far from doing this. The possibility should be taken seriously, however. Black holes are attractive dark-matter candidates. Primordial black holes present as halo objects could conceivably be detected by microlensing (Minty et al 2001). If one could get a lower bound on the number of black holes in this or a similar way, and get an incompatible upper bound from gamma-ray observations or cosmology, one might be able to rule out black-hole radiation experimentally.

\section{Conclusions}

The introduction to this paper listed six main conclusions, which are recapitulated in the appendix following, along with references to the places in the text which support them. The present section gives a more narrative account.

It seems unlikely we will have a convincing theoretical answer to the question "Do black holes radiate?" soon. General dimensional arguments, like that of Bekenstein and Mukhanov (1995), show that quantum-gravitational effects may enter essentially, so, without an accepted theory of quantum gravity (at least in the regimes necessary for treating the Hawking model), we cannot expect a definitive resolution.

While we may not come to an answer to this question soon, we may expect to learn a great deal from theoretical investigations, especially those attempting to model as explicitly as possible physical processes related to the Hawking mechanism. The work of Unruh (1976) and of Unruh and Wald (1982) is an example of how much can be learned by making models of the elements of processes which cannot, in the present context be taken for granted. For such models to contribute to resolving the quantumgravitational issues, they will presumably have to take up issues involving limitations on the measurability of space-time quantities.

More work needs to be done to understand the models with non-standard propagation of the quantum fields (such as Unruh's dispersive propagation). We need to know whether such models could be used to provide a solution of the trans-Planckian problem (at present, they address the problem of trans-Planckian frequencies but not that of trans-Planckian wave-numbers). We also need to understand just what aspects of these models are essential to producing thermal spectra. Also an open question is how quantum-gravitational concerns (like the one raised by the Bekenstein-Mukhanov analysis) might be resolved by such models.

The connection between black holes and thermodynamics remains inadequately understood. The Hawking process would explain the temperature of a black hole but not, of itself, explain the nature of black hole entropy. Just what black hole entropy 
is, and how it relates to other notions of entropy, remain matters of speculation. The main attempt to link the two is the generalized second law. There are some questions about the motivation for this, as well as difficulties in formulating it precisely, and there is not at present agreement about whether one needs to understand limitations on the entropy-content of matter in order to have an adequate treatment.

We cannot verify the generalized second law in its strongest form for the Hawking process, because the accounting of entropy which would be required is extremely fine. (The entropy typically changes by about one unit when a Hawking quantum is emitted, yet the absolute entropies involved are very large, and indeed, divergent in the case of the quantum field before regularization.) With some apparently reasonable approximations, however, the Hawking effect has been shown to respect the generalized second law. There are also reasonably good indications that the quantum GerochWheeler process will respect the generalized second law, although this is a case where it seems a more delicate physical analysis is needed to settle the question.

Quantum-gravitational models have had mixed results in supporting Hawking's predictions. Some dilaton models have confirmed them, but others have not. The Bekenstein-Hawking entropy formula has been confirmed for near-extremal black holes by a mode-counting calculation in string theory, but so far that theory does not provide a detailed model in which one can see that the holes do radiate. (Extremal black holes have zero temperature.) Ashtekar's "loop quantum gravity" program has established the general relation $S \propto A$ for spherically symmetric holes, again by a mode-counting argument. One would hope this program could be developed to the point where the emission of quanta and the resolution of the trans-Planckian problem understood.

A major problem is to understand what a radiating black hole really would look like (under the assumption that Hawking's mechanism is correct). Self-interactions in the quanta could lead to (at least partial) thermalization at large (compared to $R_{\text {Sch }}$ ) distances from the hole, lowering its temperature considerably; there is also the question of reliably computing how many particles of each species are produced. This is a difficult problem involving the dynamical behavior of non-linear realistic field theories at high temperature. But only when we have an idea of what the answer is will we be able to assess any experimental evidence. The answer to this problem is also important insofar as the Hawking mechanism is used to constrain the numbers of black holes in cosmological models.

While this review has been limited to black-hole physics, one should bear in mind that closely related issues arise in inflationary theories (e.g. Brandenberger 2002); one could hope for some cross-fertilization of ideas.

From an astrophysical point of view, the limitations that have been placed on cosmological models by assuming that black holes do radiate should be considered tentative, and the consequences of not making this assumption should be given comparable weight. In particular, serious consideration should be given to the possibility that "mini" black holes may contribute to the dark matter in the Universe. 
Astrophysical studies constraining the possible number of "mini" black holes by means independent of the assumption that they radiate would be most welcome.

\section{Acknowledgments}

I am grateful to many colleagues for their comments on aspects of these ideas. I would like particularly to mention Jacob Bekenstein, Steven Carlip, Robert Geroch, Theodore Jacobson, Don Page, William Unruh and Robert Wald. Needless to say, the perspective and judgments in this paper are my own.

\section{Appendix. Text passages supporting the conclusions}

The introduction listed six main conclusions of this paper. This appendix contains, for each conclusion, a list of the places in the text where the arguments supporting it are given.

(a) All derivations of Hawking radiation involve speculations of what physics is like at trans-Planckian scales.

For the Hawking model, see section 3, especially 3.5.2 (how trans-Planckian field modes enter), and section 4 (the trans-Planckian problem generally). For the responses to arguments based on the Unruh process, see section 5.2 (the arguments need as input the ultraviolet behavior of the Hawking state). For models with nonstandard propagation, see 8.2.2 and 8.4 (ultra-high wave-numbers enter essentially).

Moving-mirror models and 't Hooft's model do not really derive Hawking radiation; for comments on these, see the introduction to section 6 , and 9.1.3.

Of course, arguments bases on theories of quantum gravity (section 10) all involve, at least implicitly, speculations about Planck-scale physics.

(b) There are equally plausible speculations about physics at such scales which result in no radiation at all, or in non-thermal spectra.

There is some question here of when two speculations are equally plausible, of course. What I list here corresponds to ideas which either have been taken seriously by respected workers, or fall within a reasonable conservative physical judgement.

See the last paragraph of 3.5.2 (a textbook-style cut-off theory would lead to no Hawking radiation), the last paragraph of 8.1 (Jacobson's analysis led him to think there might be modifications to the Hawking spectrum), section 10.1 (some dilaton models reproduce Hawking radiation, some do not), section 11.2 (quantization of black-hole area could alter or obviate the production of Hawking radiation).

(c) The various derivations which have been put forward are not all mutually consistent.

Two models which could in principle be experimentally distinguished will be considered inconsistent (although they may agree in some regimes, e.g., they may both produce the Hawking spectrum at large distances). 
The models with non-standard propagation (section 8) evidently differ essentially from the original Hawking model (section 3). 't Hooft's model probably differs essentially from Hawking's near the horizon (section 9.1.3, cf. also section 9.1.4).

(d) Quantum-gravitational corrections are very plausibly of a size to alter or even obviate the prediction of thermal radiation.

Note that the assertion is not that quantum-gravitational corrections do alter Hawking's prediction, but that they are of a size where they plausibly might.

See the end of section 6.2 (moving-mirror models suggest quantum-gravitational corrections might be significant), section 10.1 (dilatonic models might or might not reproduce Hawking radiation, depending on how they are tweaked), and section 11, especially 11.2 (quantization of black-hole area could lead to alteration of Hawking's predictions).

(e) A number of arguments which have been put forward in support of the Hawking mechanism are not really direct evidence for the existence of thermal radiation, but rather are arguments for interpreting black holes' areas as entropies.

See the introduction to section 7 (black-hole thermodynamics generally), and sections 10.2-10.4 (mode-counting arguments from quantum gravity theories).

(f) The proposed mechanism, at least as conventionally understood, relies precisely on the assumption that quantum-gravitational effects can be neglected, and so no deep test of quantum gravity can emerge from it.

The second part of this assertion follows from the first. For the first part, the Hawking model's classical treatment of space-time at all scales is emphasized in section 3.5.2. Discussion of the neglect of quantum-gravitational effects will be found at the end of section 6.2 (parallels suggested by moving-mirror models) and especially in sections 11.1 (limits to the validity of semi-classical treatments), 11.3 (possible quantum-measurement theoretic limitations).

\section{References}

Amelino-Camelia, G 2002 Int J Mod Phys D11 35-60 gr-qc/0012051

Amelino-Camelia, G, Ellis, J, Mavromatos, N E, Nanopoulos, D V and Sarkar, S 1998 Nature 393 763-765 astro-ph/9712103

Ashtekar, A 1998 The geometric universe: science, geometry and the work of Roger Penrose (Oxford: University Press), eds. Huggett, S A, Mason, L J, Tod, K P, Tsou, S T and Woodhouse, N M J, pp 173-194

Ashtekar, A, Baez, J C, and Krasnov, K 2000 Adv Theor Math Phys 4 1-94

Balbinot, R and Fabbri, A 1999 Phys Lett B459 112

Bekenstein, J D 1972a Phys Rev D5 1239

— 1972b Phys Rev D5 2403

1973 Phys Rev D7 2333-2346

1975 Phys Rev D12 3077-3085

1982 Phys Rev D26 950

1994a The Seventh Marcel Grossmann Meeting on recent developments in theoretical and experimental general relativity, gravitation, and relativistic field theories: Proceedings (World Scientific), eds Robert T Jantzen, G Mac Keiser, and Remo Ruffini, pp 39-58

— 1994b Phys Rev D49 1912 
1999 Phys Rev D60 124010

2002 in Advances in the interplay between quantum and gravity physics eds P G Bergmann and V de Sabbata (Kluwer), pp 1-26, gr-qc/0107049

Bekenstein, J D and Mukhanov, F F 1995 Phys Lett B360 7-12

Belinski, V A 1995 Phys Lett A209 13-20

Birrell, N D and Davies, P C W 1982 Quantum fields in curved space (Cambridge: University Press)

Bisognano, J J and Wichmann, E H 1976 J Math Phys 17303

Błaut, A, Kowalski-Glikman, J and Nowak-Szczepaniak, D 2001 Phys Lett B521 364-370 grqc/0108069

Bombelli, L, Koul, R K, Lee, J and Sorkin, R D 1986 Phys Rev D34 373-383

Boulware, D G 1975 phys Rev D11 1404

Bousso, R 2002 The holographic principle, submitted to Rev Mod Phys hep-th/0203101 52 pp

Brandenberger, R H 2002 Trans-Planckian physics and inflationary cosmology, hep-th/0210186

Brout, R, Massar, Parentani, R and Spindel, Ph 1995a Phys Rep 260 329-454 1995b Phys Rev D52 4559-4568, hep-th/9506121

Buckley, J et al 2002 Gamma-ray summary report (Snowmass 2001) astrop-ph/0201160

Carlip, S 2001 Rept Prog Phys 642001

Cline, J M, Mostoslavsky, M and Servant, G 1999 Phys Rev D59 063009

Corley, S and Jacobson, T 1996 Phys Rev D53 6720-6724, hep-th/9601073

Daghigh, R G and Kapusta, J I 2002 Phys Rev D65 064028, |gr-qc/0109090

Das, S, Ramadevi, P and Yajnik, U A 2002 Mod Phys Lett A17 993-1000

Davies, P C W and Fulling, S A 1977 Proc R Soc Lond A356 237

DeWitt, B S 1979 in General relativity: an Einstein centenary survey eds S W Hawking and W Israel (Cambridge), pp 680-745

Eddington, A S 1929 The nature of the physical world (New York: MacMillan and Cambridge: University Press)

Fedotov, A M, Mur, V D, Narozhny, N B, Belinskii, V A and Karnakov, B M 1000 Phys Lett A254 $126-132$

Fredenhagen, K and Haag, R 1990 Commun Math Phys 127273

Frolov, V P and Page, D N 1993 Phys Rev Lett 71 3902-3905

Fulling, S A and Davies, P C W 1976 Proc R Soc Lond A348 393

Gibbons, G W 1977 in Proc. First Marcel Grossman Meeting on General Relativity, ed. R. Ruffini $449-458$

Gibbons, G W and Hawking, S W 1977 Phys Rev D15 2752

Grumiller, D, Kummer, W and Vassilevich, D V 2002 Phys Rept to appear

Halzen, F, Zas, E, MacGibbon, J H and Weekes, T C 1991 Nature 353 807-815

Hawking, S W 1974 Nature 24830

1975 Commun Math Phys 43 199-220

1976 Phys Rev D13 191-197

Heckler, A F 1997 Phys Rev Lett 78 3430-3433

Helfer, A D 1996 Class Q Grav 13 L129-L134

1998 Class Q Grav 15 1169-1183

— 2001a Phys Rev D63 025016

— 2001b Class Q Grav 18 5413-5428

2003 Quantum energetics of the Hawking process, in preparation

Himemoto, Y and Tanaka, T 2000 Phys Rev D61 064004 gr-qc/9904076

Hod, S 2000 Phys Rev D61 124016

't Hooft, G 1985 Nucl Phys B256 727

1993 in Salamfestschrift: A collection of talks eds. A. Ali, J. Ellis, S. Randjbar-Daemi, World Scientific, pp 284-296, gr-qc/9310026

1996 Int J Mod Phys A11 4623-88, gr-qc/9607022

1997 Proceedings: Twelfth International Congress of Mathematical Physicists (ICMP 97) eds D

De Wit, A J Bracken, M D Gould and P A Pearce, International Press, pp 64-79, gr-qc/9711053

1999 in Basics and highlights in fundamental physics (Erice 1999) ed. A Zichichi, World Scientific, pp 72-86, hep-th/0003004

Itzhaki, N 1996 Some remarks on 't Hooft's S-matrix for black holes hep-th/9603067 
Jacobson T 1990 Brighton 1990: Relativistic astrophysics, cosmology and fundamental physics (proc. 15th Texas symposium on relativistic astrophysics 104-116

1991 Phys Rev D44 1731-1739

1993 Phys Rev D48 728-741

1996 in Recent developments in gravitation and mathematical physics: proceedings [First Mexican school on gravitation and methamtical physics], eds. Macias, A, Matos, T, Obregon, O and Quevedo, H, World Scientific, pp 87-114 1999 Prog The Phys Suppl 136 1-17, hep-th/0001085

Jacobson, T and Mattingly, D 2000 Phys Rev D61 024017

Kapusta, J I 2000 in Phase transitions in the rarly universe: theory and observations (International School of Astrophysics D. Chalonge, Erice 2000) eds H J de Vega, I Khalatnikov, N Sanchez, Kluwer Academic, astro-ph/0101515

Kim, H I, Lee, C H and MacGibbon, J H 1999 Phys Rev D59 063004

Kummer, W and Vassilevich, D V 1999 Annalen Phys 8 801-827

Marolf, D and Sorkin, R D 2002 Perfect mirrors and the self-accelerating box paradox preprint hepth/0201255

Minty, E M, Heavens, A F, and Hawkins, M R S 2001 Testing dark matter with high-redshift supernovae astro-ph/0104221

Page, D N 1976 Phys Rev D14 3260-3273

Panangaden, P and Wald, R M 1977 Phys Rev D16 929-932

Parentani, R 1996 Nucl Phys B465 4526

2001 Phys Rev D63 041503

Peet, A 2000 Boulder 1999: Strings, branes and gravity (TASI lectures), pp 353-433

Penrose, R 1986 in Quantum concepts in space and time (Oxford, 1984) eds R Penrose and C J Isham (Oxford) pp.129-146

Penrose R and Rindler W 1984-6 Spinors and Space-Time (Cambridge: University Press)

Radzikowski, M J and Unruh, W G 1988 Phys Rev D37 3059-3060

Saida, H and Sakagami, M 2000 Phys Rev D61 084023 gr-qc/9905034

Schweber, S S 1961 An introduction to relativistic quantum field theory (Evanston, Illinois: Row, Peterson and Co.)

Sewell, G 1982 Ann Phys 141 201-224

Smolin, L 2001 Nucl Phys B601 209 hep-th/0003056

Susskind, L, Thorlacius, L and Uglum, J 1993 Phys Rev D48 3743-3761, hep-th/9306069

Unruh, W G 1976 Phys Rev D14 870-892

1981 Phys Rev Lett 46 1351-1353 1995 Phys Rev D51 2827-2838

Unruh, W G and Wald, R M 1982 Phys Rev D25 942-958

Visser, M 2001 hep-th/0106111, Essential and inessential features of Hawking radiation

Wald, R M 1994 Quantum Field Theory in Curved Spacetime and Black Hole Thermodynamics (Chicago: University Press)

_2001 Living Rev Rel 46 